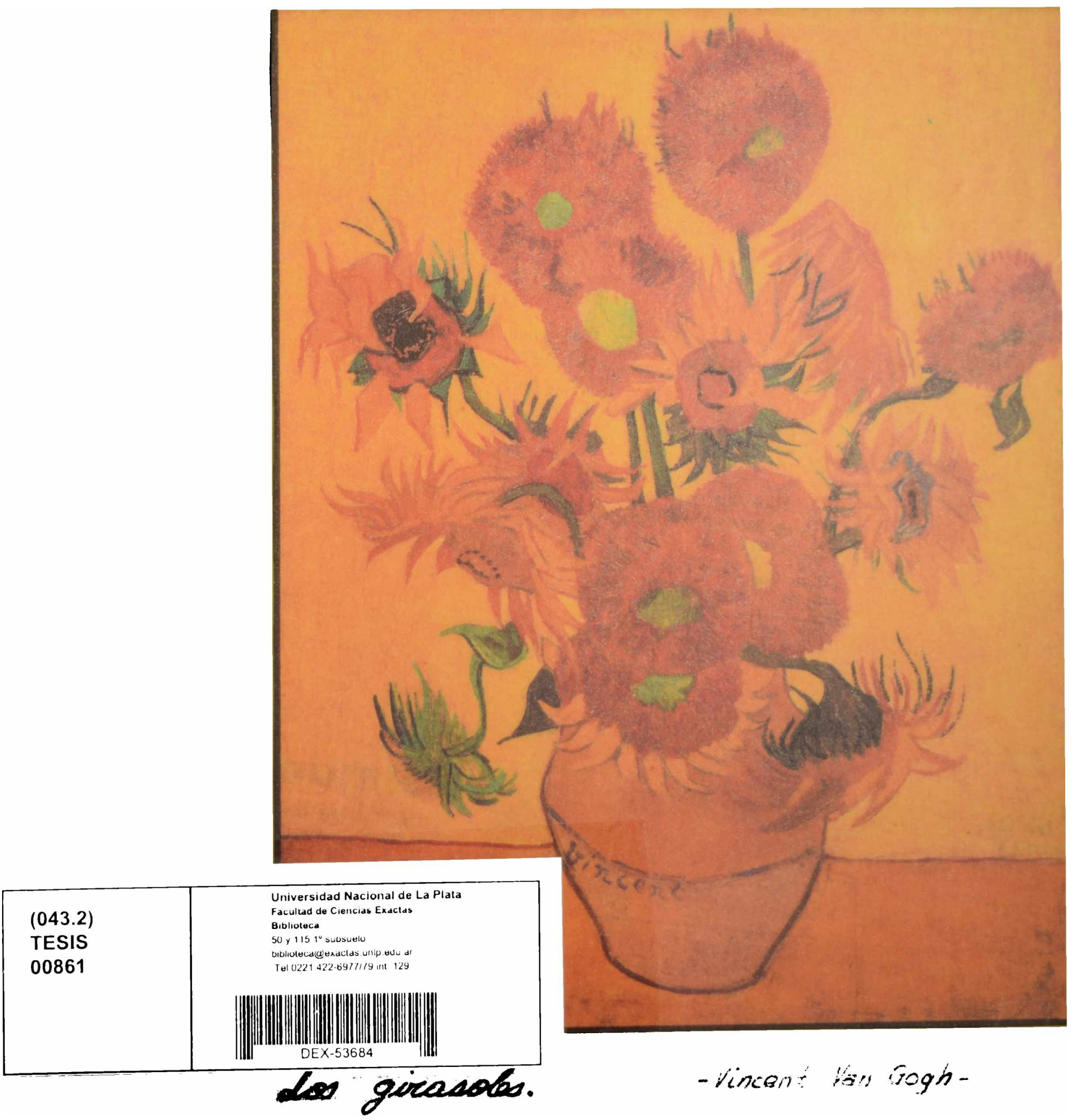


UNIVERSIDAD NACIONAL DE LA PLATA

FACULTAD DE CIENCIAS EXACTAS

CRISTALIZACION DE TRIGLICERIDOS

EN MEZCLAS COMPLEJAS

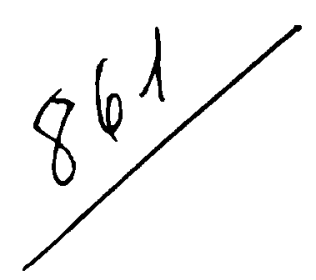

MARIA LIDIA HERRERA

TESIS

1990

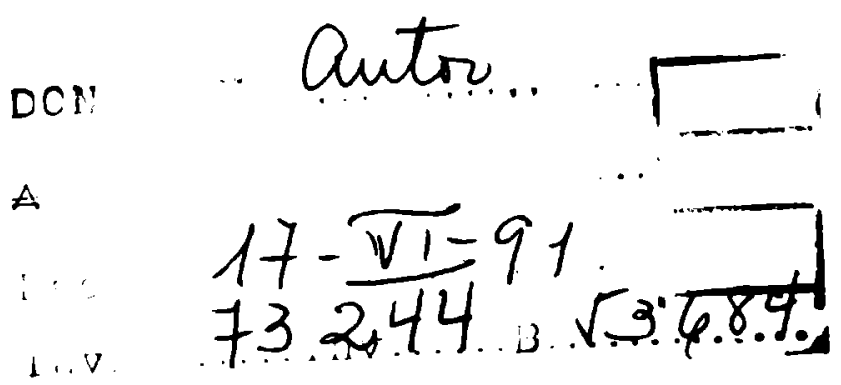


El presente trabajo de tesis para optar al titulo de Doctor en Ciencias Bioguímicas, fue realizado en el Centro de Investigación y Desarrollo en Criotecnologia de Alimentos, Facultad de Ciencias Exactas de la Universidad Nacional de La Plata, bajo la direcclón de la Doctora Maria Cristina Añon. 
Al Consejo Nacional de Investigaciones Cientificas y Ténicas (CONICET) por haberme dado la posibilidad de emprender y desarrollar este trabajo de investigación a través de las becas que me fueron otorgadas.

A la Facultad de Ciencias Exactas de la Universidad Nacional de La Plata por la formación cientifica y humana que me brindó.

A la Doctora Maria Cristina Anón por haber posibilitado mi iniciación en la lnvestigación, brindándome su guia, dedicación y estimulo durante la dirección de este trabajo.

Al Ingeniero Juan Alejandro Segura por el desarrollo de la tecnologia que hizo posible estas experiencias y por el sólido apoyo y marco conceptual que me brindó durante el desarrollo de este trabajo de tesis.

A Molinos Rio de La Plata S.A. por haber aportado todas las muestras que fueron empleadas en estas experiencias.

A Ia Doctora Marta Melgarajo por la gentileza con que fui recibida en su laboratorio.

Al señor Walter Atencio por su amistad y apoyo técnico.

Al seffor Sergio Torres por su vallosa ayuda en la construcción del equipo fraccionador. 
Al señor Arturo Colavita por el aporte de ideas en la corrección del diseño del equipo fraccionador.

A la señora Silvia Garcia por su apoyo técnico en la obtención de los diagramas de Rayos $X$.

Al Ingeniero Nestor Serena por su esmerada labor en la calibración del sistema de control de temperatura.

Al Ingeniero Santiago Klappenbach por su colaboración en la puesta en marcha del equipo fraccionador.

A mis compañeros del CIDCA por su amistad y su apoyo y a todos los que, de alguna manera, contribuyeron a la concreción de este trabajo. 
"El orden que imagina nuestra mente es como una red, o una escalera, que se construye para llegar hasta algo. Pero despues hay que arrojar la escalera, porque se descubre que, aunque haya servido, carecta de sentido."

El nombre de la rosa, Umberto Eco 
INDICE 
1. Caracteristicas generales de grasas y aceites 1

1. 1 Importancia comercial 1

1.2 Importancia nutricional 2

2. Operación de cristalización 4

2.1 Etapas involucradas 4

2.2 Polimorfismo 9

2.3 Cristalización de soluciones y aleaciones 23

2.4 Cristalización de grasas y aceites 25

3. Hodificación de grasas y aceites 30

3.1 Hidrogenación $\quad 32$

3.2 Interesterificación 36

$\begin{array}{lll}3.3 & \text { Eraccionamiento } & 37\end{array}$

$\begin{array}{lr}\text { OBJET I VOS } & 43\end{array}$

MA'ERIALES Y METUDOS

1. Muestra enpleada 44

2. Fraccionaniento 44

2.1 Equipo empleado $\quad 44$

$\begin{array}{ll}2.2 \text { Método utilizado } & 47\end{array}$

$\begin{array}{lll}2.3 & \text { Prueba piloto } & 47\end{array}$

3. Deterninación cuantitativa de acidos grasos por cronatografia $\begin{array}{lr}\text { gaseosa (GLC) } & 49\end{array}$

3.1 Preparación de la muestra 48

3.2 Equipo utilizado $\quad 49$

3.3 Condiciones de corrida 48

4. Determinación cuantitativa de triglicéridos por cronatografia liquida de alta presión (HPLC) 50

$\begin{array}{ll}4.1 \text { Preparacion de la ruestra } & 50\end{array}$ 
4.2 Equipo utilizsdo

4. 3 Condiciones de corrida

4.4 Cuantificación de las áreas e identificación de componentes 52

5. Calorinetria diferencial de barrido (DSC)

5.1 Equipr utilizado

5.2 Calibración del equipo 53

5.3 Condiciones de corrida 55

5.4 Interpretación de los termogramas 55

5. C Cálculo del calor de transición 56

6. Difractometria de Rayos $X$ 57

6.1 Equipo empleado 57

6.2 Condiciones de corrida 57

6.3 Interpretación de los diagramas 58

7. Espectroscopia infrarroja 59

7. 1 Preparación de 1 a muestra

7.2 Equipo utilizado 59

7.3 Condiciones de corrida 59

7.4 Interpretación de los diagramas 59

8. Resonancia nagnética nuclear (RMN)

8.1 Preparación de la muestra

8.2 Equipo empleado

8.3 Condiciones de corrida

\section{RESULTADOS Y DISCUSION}

1. Experiencias previas al fraccionaniento

2. Fraccionamiento de aceite de girasol hidrogenado en ausencia de solventes

2.1 Caracterización de las muestras originales

2.1.1 Determinación cuantitativa de ácidos grasos por 
2.1.2 Determinación cuantitativa de triglicéridos por cromatografia liguida de alta presión 70

2.1.3 Calorimetria diferencial de barrido 79

2.1.4 Difractometria de Rayos X 85

$\begin{array}{lll}2.1 .5 & \text { Espectroscopia infrarroja } & 87\end{array}$

2.2 Caractorización de las fracciones sólidas 89

2.2.1 Determinación cuantitativa de ácidos grasos por $\begin{array}{ll}\text { cromatografia gaseosa } & 89\end{array}$

2.2.2 Determinación cuantitativa de triglicéridos por cromatografia liguida de alta presión 92

2.2.3 Resonancia magnetica nuclear 103

2.2.4 Calorimetria diferencial de barrido 104

2.25 Difractometria de Rayos X 109

2.2 .6 Espectroscopia infrarroja 113

2.3 Caracterización de las fracciones liquidas 115

2.3.1 Determinación cuantitativa de ácidos grasos por

cromatografia gaseosa 115

2.3.2 Determinación cuantitativa de triglicéridos por

$\begin{array}{lr}\text { cromatografia liquida de alta presión } & 117\end{array}$

2.3.3 Resonancia magnética nuclear 121

2.3.4 Calorimetria diferencial de barrido 122

$\begin{array}{lr}2.3 .5 \text { Espectroscopia infrarroja } & 128\end{array}$

3. Equilibrios sólido- liquido 131

4. Influencia de la velocidad de enfrianiento 137

$\begin{array}{lr}\text { CONCLUSIOHES } & 149\end{array}$

BIBLIOGRAFIA 
P ácido palmítico

o ácido oleico

$\mathrm{S}$ ácido esteárico

$L$ ácido linoloico

Ln ácido linolénico

Pt ácido palmitoleico

A ácido araquidico

min minutos

seg segundos

m metros

cm centinetros

mn milimetros

Psi libras fuerza/pulgada cuadrada

ml nililitros

SSO diestearoleina

soo dioleoestearina

POO dioleopalmitina

000 trioleina

LOO dioleolinoleina

LLP dilinoleopalnitina

PPP tripalnitina

SSS triestearina

LLL trilinoleina

POP dipalnitoleina

PSP dipalnitoestearina

MMM triniristina

AAA triaraquidina 
ESS diestearoelaidina

POS palmitoestearoleina

sos diestearoleina

EEE trielaidina

SEE dielaidinestearina

OPO dioleopalmitina 
Introduccion 


\section{CARACTERISTICAS gEHERALES DE gRASAS Y ACEITBS}

\subsection{Importancia conercial}

Desde el punto de vista guínico las grasas son ésteres del glicerol y ácidos grasos. Las grasas enpleadas para consumo humano son mezclas de diferentes sustancias: pueden contener ácidos grasos libres, mono y diglicéridos, proteinas, vitaminas, antioxidantes, pigmentos, savorizantes, sales minerales e incluso en los productos manufacturados pueden formar emulsiones acuosas. Las grasas son llamadas aceites cuando son liquidas a temperatura ambiente, alrededor de $25^{\circ} \mathrm{C}$. La mayoria de las grasas vegetales y marinas se encuentran dentro de esta categoria. Sin embargo las grasas de palma, coco y nuez de palma son sólidas a esta temperatura. Algunas grasas vegetales como la de cacao se llaman mantecas por su aspecto semejante a este producto de origen animal.

En algunas zonas templadas y frias de Europa y Estados Unidos el tipo de producto graso más utilizado por el público es el aceite para ensalada (Schwitzer, 1951). En estas regiones los aceites tambien son extensamente usados en la industria para el fritado, para conservar sardinas, anchoas, pescados y productos cárneos. En el mediterráneo y regiones de clima cálido se usan principalmente para cocinar aceites de algodón, girasol, soja y oliva. Con igual fin, el aceite de girasol es muy emploado en algunos paises orientales, en el este de Europa asi cono tambien en Estados Unidos.

El girasol es originario de América Central y se utilizó por 
primera vez para obtener aceite en Rusia (Diccionario Enciclopédico Monitor, 1960). El cultivo de girasol ha cobrado gran importancia en estos últimos años. La producción mundial estimada do semilla de girasol en el ciclo $89 / 90$ es de 21,8 millones de toneladas según datos aportados por la Bolsa de Cereales de Buenos Aires con un aumento de 1,8 millones de toneladas respecto del ciclo previo. La conjunción de excelentes rindes y la decidida expansión de las siembras en los tres paises productores más inportantes, Unión Soviética, Argentina y Francia contribuyó al aumento de la oferta total. En la Argentina, segundo productor mundial, la mayor rentabilidad que ofrecen las oleaginosas está marcando una derivación de áreas en favor de las mismas. En el periodo mencionado la producción girasolera resultó de 3,9 millones de toneladas. El girasol tiene dos destinos principales: la producción de aceite y la producción de harinas. La producción mundial de aceite (ciclo 89/90) resultó ser de 7,6 millones de toneladas un $7,8 \%$ mayor que en el ciclo previo y la de harina $8,8 \mathrm{mi-}$ llones de toneladas (Bolsa de Cereales de Buenos Aires, 1990). El aceite a su vez, puede ser empleado para la preparación de productos como la mayonesa o ser modificado por ejemplo hidrogenado y utilizado en la preparación de materias grasas denominadas shortenings $y$ en margarinas. Los dos aceites más empleados en Argentina con este fin son el de Birasol y el de soja.

\subsection{Importancia Natricional}

Los lipidos permiten al organismo viviente un almacenamiento 
y transporte económico de la energia. Por otra parte son importantes por las vitaminas liposolubles que contienen y por los ácidos grasos esenciales que aportan. $25-35 \%$ de las calor las totales requeridas por un individuo normal deben provenir de los lipidos. Al combustionarse un gramo de grasa se liberan 9,0 Kcal. Prote1nas y carbohidratos liberan sólo $4,0 \mathrm{Kcal} / \mathrm{g}$. Aun conociendo la importancia de los lipidos, el consumidor no tiene en cuenta estas afirmaciones. Lo que le interesa conciente o inconcientemente son las reacciones sensoriales. El sentido del gusto es capaz de distinguir sabores ácidos, dulces, amargos y salados y el tacto bucal diferencias de temperatura, viscosidad, estado granulométrico, resistencia a la deformación de los alimentos, etc. Desde el punto de vista del gusto las moleculas de ácidos grasos son neutras. En efecto tienen un peso molecular elevado y son poco polares, poco solubles en la saliva y no penetran en las papilas gustativas para impresionarlas. Son poco volátiles y no llegan a las extremidades del nervio olfativo en el fondo de las fosas nasales. Pero desde ol punto de vista tactil somos sensibles a la viscosidad de los aceites y emulsiones, a las diferencias de temperatura provocadas por la fusión de las grasas que intercambian su calor latente de cambio de estado con la lengua y paladar, a la deformación de los cuerpos grasos sólidos o semisblidos. Apreciamos por ejemplo la untuosidad de una mayonesa, la frescura de una manteca o de una margarina que funde en la boca, el crocante de una barra de chocolate en los dientes. La demanda de grasas sólidas es superior a la de grasas liquidas; sin enbargo en la naturaleza se encuentra nayor cantidad de aceites. 


\section{OPERACION DE CRISTALIZACION}

\subsection{Btapas involucradas}

Cuando se calienta un sólido cristalino puro se alcanza una temperatura a la cual cambia a liquido; este es el denominado punto de fusión, que tiene un valor definido dependiente de la presión externa. Si se enfria el liquido, se produce la solidificación a la misma temperatura para una presión dada; por tanto, en una sustancia pura el punto de fusión y el de congelación son idénticos. Es importante distinguir agui los fenómenos de criatalización y de fusión. La cristalización se puede comparar a una reacción quimica en el sentido que una cierta barrera energética debe ser franqueada para permitir la formación de agregados moleculares que se transforman en cristales cuando alcanzan un tamaño critico. Se necesita un sobreenfriamiento porque este primer estadio requiere una gran energia de activación. La fusión de un cristal se produce espontáneamente sin esperar que un nivel energético suficiente para desagregarlo sea alcanzado. El sobrecalentamiento en el cambio de sólido a liquido sólo se ha observado en determinados polimeros en los que el proceso de fusión implica posiblemente la ruptura de enlaces (Glastone, 1979).

El proceso de cristalización es un fenómeno brusco. So pueden distinguir en él dos etapas: la nucleación y el crecimiento de los cristales. La nucleación es el proceso de formación de los agregados moleculares que darán origen al cristal. Por debajo de la temperatura de fusión, Tf, el pasaje de átomos de la faso li- 
guida a la sólida disminuye la energia del sistema. Sin embargo la solidificación no comienza espontáneamente a temperaturas inmediatamente inferiores a Tf ya gue es necesario primero crear una interfase que exige un aumento de energia del sistema. Cuando se forma un núcleo sólido esférico de radio, r, la disminución de energia libre del sistema debido al cambio de fase es:

$\Delta F_{1}=-4 / 3 \pi r^{3} \Delta E_{v}$

donde:

$\Delta F_{v}$ : variación de energla libre por unidad de volumen que solidifica.

en tanto que el aumento de energla libre debido a la creación de la superficie del núcleo es :

$\Delta F_{2}=4 \pi r^{2}$ o

donde:

o : energia superficial unitaria.

La variación total de energia es:

$\Delta F=\Delta F 1+\Delta F 2$

o sea que:

$\Delta F=-4 / 3 \pi r^{3} \Delta F v+4 \pi r^{2} \sigma$

Hay un radio r* minimo por encima del cual los núcleos ton- 
derán espontáneamente a crecer ya que ese proceso disminuye la energla del sistema. Núcleos de radio menor que r* tienden espontáneamente a disolverse. A medida que disminuye la temperatura (o aumenta el sobreenfriamiento) el tamaño minimo que debe tener un núcleo para crecer espontáneamente disminuye.

Para ejemplificar lo que ocurre durante la nucleación tomemos el caso de los metales liquidos los cuales están formados por poqueños agrupamientos de átomos con estructura semejante al metal sólido. Estos agrupamientos son los que actúan como núcleos. Para cada temperatura hay un tamaño "máximo razonable" de agrupamiento que puede existir. Para que los nucleos crezcan este tamaño debe ser igual a $r *$. Esta nucleación que ocurre en el seno del liquido se llama homogénea $y$ es un caso simple que sirve para comprender el problema. Solo se produce en condiciones controladas en laboratorio. En condiciones ordinarias, los núcleos se forman en las paredes del recipiente en que está contenido el líquido o sobre particulas extrañas. Esto disminuye el grado de sobrenfriamiento necesario para formar núcleos. Este tipo de nucleación se llama heterogénea.

Los constituyenteg principales de las materias grasas son los triglicéridos. A pesar del número relativamente restringido de ácidos grasos que los forman tienen una gran variabilidad de composición a consecuencia de la isomeria de posición de las cadenas de los ácidos grasos sobre el glicerol. La interacción entre los triglicéridos es uno de los factores esenciales en el comportamiento de las materias grasas en la cristalización y fusión. Las propiedades fisicas, las temperaturas de fusión, la estabilidad de los crietales, su aspecto, la velocidad de crecimiento dependen 
entre otras cosas de la naturaleza de los componentes, del aporte energetico, de la historia anterior de los triglicéridos y de la mezcla de materias grasas. Cuando los trigliceridos cristalizan aparecen arreglos moleculares regulares que marcan la formación de ligaduras más estrechas que en el estado liquido. Se observa una liberación de energia en el curso del fenómeno ( $15 \mathrm{Kcal} / \mathrm{mol}$ para triestearina, para citar una magnitud). La sobresaturación creada en la solución provoca la formación de pequeños cristales que están en equilibrio en la solución a una temperatura dada Te (Van Den Tempel, 1868; Knoester y col., 1968). La ecuación que interpreta este fenómeno es la siguiente:

$$
2 \text { o V TC }
$$

$$
\begin{aligned}
& \Delta \mathrm{T}=\mathrm{T} \mathrm{c}-\mathrm{T}=\cdots \cdots \\
& \mathbf{r} \Delta \mathrm{H} \\
& 2 \sigma \mathrm{V} \quad \mathrm{Cr} \\
& \text { y } \\
& \text { r } \quad \text { C }
\end{aligned}
$$

donde :

Tc : temperatura de sobresaturación $\left({ }^{\circ} \mathrm{C}\right)$

$\mathrm{T}$ : temperatura operacional $\left({ }^{\circ} \mathrm{C}\right)$

$\sigma$ : tensión interfacial crigtal solución (ergs/cm ${ }^{2}$ )

$\mathrm{V}$ : Volumen molar del compuesto cristalizado ( $\mathrm{cm}^{3} / \mathrm{mol}^{\text {) }}$

$r$ : radio del cristal (cm)

$\Delta H$ : calor de fusión (erg/mol)

Cr : Concentración de los cristales a la temperatura de 
sobresaturacion ( nol/ $\left.\mathrm{cm}^{3}\right)$

C : concentración de equilibrio de los cristales en la solución $\left(\operatorname{mol} / \mathrm{cm}^{3}\right)$

La segunda etapa de la cristalización, el crecimiento de los cristales, depende de la sobresaturación. Para pasar de un estado de disperaión aleatoria ana disperaion ordenada es necosario que las moléculas tengan tiempo de desplazarse. Un enfriamiento intenso provoca un cambio de estado mas rápido y puede dificultar la cristalización sea porque las moléculas no tienen la movilidad suficiente sobre todo en un medio en el cual la viscosidad es Erande, sea porque las moléculas no tienen el tiempo necesario para cambiar de estado. De aqui que se obtenga un estado vitroso. Hay una competencia entre la velocidad con que las moléculas pueden desplazarse y la incorporación a la red cristalina, de manera que la velocidad de crecimiento de los cristales pasa por un máximo en función de la temperatura.

Cristalización $y$ fusión en el caso de las grasas no se presentan como fenómenos simples. En estos procesos influyen preponderantemente las condiciones prácticas (velocidad de transferencia de calor, materia y movimiento, gradientes de temperatura, viscosidad, agitación del medio) y las condiciones de procesamiento (procesos a que son sometidas las materias grasas en la industria) según los cambios de estado se produzcan en rangos de temperaturas más o menos amplios. Todos estos factores influyen en forma diferente sobre fusión y cristalización. 


\subsection{Polinorfismo}

Los triglicéridos al cristalizar presentan el fenómeno de polimorfismo es decir, en el estado sólido poseen más de una forma cristalina dependiendo de la velocidad de cristalización y de su naturaleza quimica. Las formas cristalinas de los triglicaridos pueden ser estudiadas por una variedad de métodos entre los que pueden citarse determinación del punto de fusión, difractometria de rayos $X$, espectroscopia infrarroja, análisis térmico diferencial, métodos microscópicos, dilatométricos y resonancia magnética nuclear.

Dos nomenclaturas son usadas en la actualidad para designar a las formas polimorficas de los triglicéridos: la propuesta por Malkin (1954) basada en el orden de los puntos de fusión (la forma de mayor punto de fusión es designada $\beta$, la de menor punto de $f u-$ sión a y la de punto de fusión intermedio $\beta^{\prime}$. Las modificaciones se designan con números romanos) y la propuesta por Lutton (1955), basada en los espaciamientos cortos que se presentan en los diagramas de rayos $X$. En este tipo de diagramas se miden dos clases diferentes de distancias; los espaciamientos cortos: distancia entre las moléculas adyacentes de una capa cristalina y los espaciamientos largos: espesor de una capa crietalina. En esta nomenclatura se usan tambien las designaciones $\alpha, \beta$ y $\beta$. La forma $a$ presenta una linea fuerte a $4,15 \mathrm{~A}$. La $\beta^{\circ}$ dos o tres lineas fuertes a 4,2 A y 3,5 A y la forma $B$ una linea fuerte a $4,6 \mathrm{~A}$, usualmente la más fuerte. En adición a estas tres formas cristalinas se han observado otras modificaciones. Larsson (1964) ha completado esta ultima nomenclatura. Cuando la forma a es enfriada las molé- 
culas so empaguetan en eatructuras más cerradas $y$ tienen menor movilidad dando lugar a una forma sub-a con un diagrama de rayos $X$ correspondiente a la forma $\beta^{\circ}$. Esta forma sub-a es llamada vitrea por Malkin y a veces tambien denominada $\gamma$ por otros autores (Suzuki y col., 1985).

Chapman (1957) sugirió que se usaran las letras $M$ o L para indicar si se trata de la nomenclatura de Malkin o la de Lutton. En el caso de PSP, la forma cristalina de mayor punto de fusión funde a $68,6^{\circ} \mathrm{C}$ y puede 1 lamarse $B M$ pero presenta en su diagrama de rayos $X$ dos picos a $3,87 \mathrm{~A}$ y 4,18 A. Según la nomenclatura de Lutton se trata de la forma $\beta^{\prime} L$. Este mismo autor ha desarrollado con posterioridad un método propio para la clasificación de los cristales de triglicéridos basado en la absorción infrarroja de las distintas formas a $720 \mathrm{~cm}^{-1}$ (Chapman, 1960). La forma sub-a presenta un doblete a 719 y $727 \mathrm{~cm}^{-1}$, la forma aL una banda simple a $720 \mathrm{~cm}^{-1}$, la forma $\beta^{\circ} \mathrm{L}$ un doblete a 719 y $727 \mathrm{~cm}^{-1}$ y la forma $\beta_{L}$ una banda simple a $717 \mathrm{~cm}^{-1}$. La transición $a \rightarrow$ sub-a es reversible, pero en las otras formas las transiciones oon en un solo sentido $a \longrightarrow \beta^{\prime} \longrightarrow \beta$. La subcelda de la forma a es hexagonal y los átomos de carbono tienen un cierto grado de movilidad. La subcelda de la forma $\beta^{\prime}$ es ortorrómbica y tienen lugar dos orientaciones de planos de cadena que son perpendiculares entre si. En la forma $\beta$ todos los planos de cadena son paralelos y la subcelda nás usual en triglicéridos es triclinica. La fase sub-a tiene menor estabilidad térmioa que la fase a y se cree que posee un empaquetamiento de cadena tipo $\beta^{\circ}$. Se han encontrado tambien fases oon empaguetamiento de cadena mezclado. Fases con el mismo tipo de empaquetamiento de cadena pero con diferente estabilidad térmica 
que tienen lugar en la misma muestra se distinguen por aubclases $\beta^{\prime} 1, \beta^{\prime} 2$, etc, en el orden de estabilidad térmica decreciente. Como resultado del tipo de empaquetamiento de las cadenas de ácidos grasos la forma más estable posee el mayor punto de fusión, la mayor densidad, la mayor dilatación y el mayor calor espeoffico. Para la forma $\beta^{-}$se suele encontrar más de un punto de fusión. Esto es debido a que la celda unidad es suficientemente larga para contener dos o tres cadenas de ácidos grasos. La presencia de doble o triple cadena puede detectarse por medio de los espaciamientos largos de los diagramas de rayos $X$.

Numerosos autores han estudiado las formas polimóricas de los ácidos grasos. Los ácidos grasos más estudiados dada su importancia son esteárico (C18:0); oleico (C18:1-cis) y erucidico (C18:1-trans). Para el ácido esteárico se encontraron dos formas polimórficas B y C que se transforman una en otra cuando se hallan en solución (Sato y col., 1985). Esta transformación ocurre cuando la forma más estable (la forma $\mathrm{C}$ por encima de $32^{\circ} \mathrm{C}$ y la forma $\mathrm{B}$ por debajo de dicha temperatura) crece a expensas de la menos estable. La velocidad con que ocurre esta transición depende del solvente y de la temperatura. La propiedad más importante a tener en cuenta en lo que al solvente se refiere es su polaridad. Garti $y$ Sato (1886) estudiaron el efecto de los detergentes en la velocidad de transición de las formas cristalinas en solución. Encontraron que algunos detergentes monoionicos retardan las transiciones $\mathrm{E}-\mathrm{C}$ o $\mathrm{C}-\mathrm{B}$, es decir entre la forma inestable y la estable del ácido esteárico en soluciones de metanol, n-hexano y decano. Este efecto depende de la naturaleza quimica del detergente y del solvente. Mediante calorimetria diferencial de barrido y difracto- 
metria de rayos $X$ se estudiaron las formas polimórficas de ácido oleico ultrapuro (99,99\%) y se las comparó con los resultados hallados para ácido esteárico (Suzuki y col., 1985). El espectro de rayos $X$ reveló tres formas cristalinas $a, \beta y \gamma$. Las formas $a y$ B mostraron concordancia con las descriptas por Lutton ( 1955 ). Coincidieron tambien con las formas polimorficas de bajo y alto punto de fusión definidas por Malkin. La forma $\gamma$ se identifico por primera vez en esta ocasión. Se corroboró ademas que la forma $\beta$ es estable mientras que las formas a y $\gamma$ son metaestables. La forma a se obtiene cristalizando a mayor velocidad que la necesaria para la obtención de la forma $\beta$. Sus puntos de fusión son $13,3^{\circ} \mathrm{C}$ y $16,2^{\circ} \mathrm{C}$ respectivamente. Es importante destacar que se puso de manifiesto aqui la importancia de las condiciones de cristalización y su estrecha relación con la forma polimórfica obtenida. De acuerdo a los estudios realizados por Sato y Suzuki (1986) la forma $\beta$ del ácido oleico es menos solubles que las formas $a y$ en acetona y decano a cada temperatura ensayada. En las cercanias de Ia saturación de la solución se observaron transiciones $\alpha \longrightarrow \beta \quad 0$ ..$\longrightarrow \beta$. Se produce nucleación de la forma $\beta$ a expensas de las formas menos estables. Los citados autores determinaron por primera vez los hábitos cristalinos de las formas describiendo los cristales $\alpha, \beta$ y $\gamma$.

Se han hecho gran cantidad de estudios de los triglicéridos puros en especial de los saturados. A modo de ejemplo citaremos los trabajos de Schlichter y colaboradores (1986) quienes mediante calorimetria diferencial de barrido de SSS determinaron los calores especificos de la forma a a 223 y $323 \mathrm{~K} \quad(0,3129$ y 0,5757 $\mathrm{ca} 1 / \mathrm{g} / \mathrm{K})$ y de la forma $\beta(0,2795$ y $0,4157 \mathrm{cal} / \mathrm{g} / \mathrm{K})$ a las mismas 
temperaturas. Hampson y Rothbart (1983) informaron valores ooncordantes con los hallados por Schlichter para ambas formas a $\mathrm{y}$. Para este mismo sistema a estas mismas temperaturas Simpson y colaboradores (1984) hallaron valores de 0,2890 y $0,3350 \mathrm{cal} / \mathrm{g} / \mathrm{R}$ para la forma a y 0,4300 y $0,5770 \mathrm{cal} / \mathrm{g} / \mathrm{K}$ para la forma $\beta$, discrepando con los dos autores anteriormente citados. Schlichter y colaboradores ( 1987 ) estudiaron además el efecto de los emulsificantes sobre el calor especifico. Para ello utilizaron Span 60 y Span 65. Los emulsificantes incrementan los calores especificos de la forma $\beta$ a partir del punto en el cual la curva de calor especlfico en función de la temperatura cambia de pendiente. Por debajo de esta temperatura los valores no se ven afectados. Para la forma a en cambio, no se observan modificaciones en los calores especificos por el agregado de emulsificantes. Los emulsificantes tienen la capacidad de retardar transformaciones polimórficas indeseables. Este efecto inhibitorio en las transformaciones do la forma $\alpha$ a la $\beta$ no es absoluto, presenta variaciones a diferentes velocidades de calentamiento. Este hecho confirma la suposición que existen mecanismos de transormacion alternativos bajo diferentes condiciones cinéticas. Walker (1987) estudio el comportamiento de SSS por calorimetria diferencial de barrido. Identifico las formas a y $\beta$ pero no pudo probar la presencia de formas $\beta^{\circ}$. En contraste con estos resultados Dafler (1977) explicó el comportamiento térmico de SSS sugiriendo que la forma a se convierte rápidamente en $\beta^{\prime} y$ esta a su vez se transforma on $\beta$ por dos mecanismos separados cada uno con una constante de velocidad diferente. Por su parte, Azoury y colaboradores (1988) estudiaron el efecto de los detergentes sólidos sobre las formas polimórficas a y $\beta$ de 
SSS efectuando medidas de resonancia magnética del protón. Encontraron que el tiempo de relajación de la forma $\beta$ se afectaba on presencia de los detergentes no asi el de la forma a. Se han degcripto para Triheptadecanoina y para SSS cuatro fases solidas a, $\beta^{\prime} 1, \beta^{\prime} 2$ y $\beta$. Las temperaturas de fusion de estas fases fueron 65 , 62,60 y $50^{\circ} \mathrm{C}$ para las fases $\beta, \beta^{\prime} 2, \beta^{\prime} 1$ y a de Triheptadecanoing y $72,64,61$ y $55^{\circ} \mathrm{C}$ para las mismas fases de SSS (Simpson, 1983). El mencionado autor concluyb ademas que las propledades polimorficas de los triglicéridos resultan del balance de tres tipos predominantes de interacciones: entre los grupos terminales planares, entre las cadenas hidrocarbonadas laterales e interacciones intermoleculares de las unidades de glicerol. Esto contradice la creencia general que las interacciones entre los grupos terminales planares dictan el comportamiento fisico de los lipidos. Simpson y colaboradores (1984) también calcularon los calores especificos para esas formas polimórficas en un rango de 190 a $320 \mathrm{~K}$. Encontraron que las fases a y $\beta$ de trimargarina y triestearina tenian valores semejantes en contraste con las formas $\beta^{\prime} 1$ y $\beta^{\prime} 2$ que presentaban valores diferentes. Asi mismo, se han estudiado las formas polimórficas de PPP y SSS obteniéndose información sobre las distintas orientaciones de las cadenas, movilidad relativa de regiones particulares de estas moléculas para las distintas fornas polimórficas (Bociek y col., 1985). Gibon y colaboradores (1986) por su lado estudiaron las formas polimórficas de PPP, PSP y POP y sus mezclas en diferentes proporciones. Estos trigliceridos permiten poner en evidencia factores como la longitud de las cadenas hidrocarbonadas ( $P$ y S) y la presencia de una insaturación sobre el polinorfismo y la intersolubilidad de estas moleculas. PPP y 
PSP presentan una temperatura de fusión comprendida entre 65 y $70^{\circ} \mathrm{C}$ mientras que $P O P$ funde alrededor de temperatura ambiente. Los estudios realizados por difractometria de rayos $X$ y calorimetria diferencial de barrido permitieron construir diagramas binarios de PPP-PSP y PPP-POP. Dichos diagramas muestran el rol que juega una doble ligadura cis en una red de cadenas saturadas. En el diagrama PPP-POP se observa la formación de una solución sólida y una transformación monotéctica (un liquido da origen a un sólido y otro liquido de composición diferente) para la forma $\beta^{\prime}$. Para el sistema saturado sólo se forma solución sólida para esta faso. Es decir que la presencia de una insaturación modifica la solubilidad de estos dos triglicéridos entre si. Las temperaturas de fusión similares de PPP y PSP asi como la saturación de las cadenas es lo que posibilita, a pesar de sus longitudes diferentes, conservar ung. solución sólida $\beta^{\prime}$ hasta la fusión total de los componentes. Sin embargo se observa una transición a dos fases cuando aparece la forma $\beta$. Los glicéridos mixtos se solidifican dificilmente bajo esta forma. Cuando los cristales de PPP, SSS y sus mezclas con 000 en diferentes proporciones se estudiaron por difractometria de rayos $X$, calorimetria diferencial de barrido y resonancia magnética nuclear se encontró que enfriando muy rápidamente las muestras, los triglicéridos cristalizaban en la forma $\alpha$. Pocos minutos después, ésta se transformaba en $\beta^{\circ}$. Luego se observaba una disminución gradual de los espaciamientos largos alcanzando los valores correspondientes a la forma $\beta$ (Norton y col., 1985). Hampson y Rothbart (1983) estudiaron por calorimetria diferencial de barrido los calores especificos de LLL, MMM, PPP y SSS al igual que Charbonnet y Singleton (1947). Estos autores encontraron valores dis- 
cordantes entre si. Todos los estudios citados anteriormente como ejemplos y muchos otros no siempre han llegado a las mismas conclusiones. Esta controversia ha influido desfavorablemente en la comprension del comportamiento de grasas y aceites.

Los resultados obtenidos con triglicéridos puros ae han volcado posteriormente en el estudio de sistemas complejos. Schlichter y colaboradores (1988) estudiaron la cristalización de manteca de cacao por calorimetria diferencial de barrido en presencia $y$ ausencia de monoestearato de sorbitan a diferentes velocidades. La velocidad de enfriamiento afecta las entalpias y temperaturas de las transiciones de fases. Una velocidad muy lenta de enfriamiento causa un descenso significativo en la entalpia de cristalización sugiriendo que el fraccionamiento de los gliceridos ocurre bajo esas condiciones. En presencia de 5\% de monoestearato de sorbitan el punto de solidificación es mayor y el de fusión más bajo. Ambas entalpias de cristalización y fusión son menores que la de manteca de cacao pura. La presencia de emulsificantes parece promover la cristalización fraccionada. Las curvas de fusión después de diferentes perlodos de cristalización isotérmica sugieren que las formas polimóricas de alto punto de fusión difieren de las de bajo punto de fusión y que la presencia de monoestearato de sorbitan afecta el fraccionamiento de grasas. Tambien se ha estudiado el efecto de los emulsificantes en las transiciones polimórficas en manteca de cacao (Garti y col., 1986) haciendo hincapié en las formas IV, $V$ y VI que son gignificativas para la industria. Los emulsificantes sólidos son los aŕs eficientes en evitar la transición de la forma $V$ a la VI. Estas formas polimórficas de la I a la VI fueron caracterizadas por Wille y Lutton en 1966. 
El polimorfismo de aceite de nabo ha sido estudiado empleando difractometria de rayos $X$ y microscopia de luz polarizada (Naguib y col., 1985). Para ello se utilizaron muestras de aceites hidrogenados de Indice de iodo 60 y 70 . Ambas muestras se hidrogenaron de manera selectiva y no selectiva. En un primer momento todas las muestras cristalizaban en la forma $\beta$. pero luefo ocurrian transiciones $\beta^{\circ} \rightarrow \beta$. La muestra hidrogenada no selectivamente presentó un comportamiento distinto según la extensión de la hidrogenación, no encontrándose diferencias en la muestra hidrogenada selectivamente. En ambos casos la hidrogenación selectiva demoró la transformación polimorfica. Las formas polimórficas se caracterizaron por microscopla teniendo como patrón SSS pura cristalizada en las formas $\alpha, \beta^{\prime}$ y $\beta$. Cuando el aceite de mayor indice de iodo se fraccionó con detergente, se lavó, se secó y se fijó se obtuvo predominantemente la forma $\beta$.

Debido a la gran expansión en los volúmenes de producción experimentada por el aceite de palma numerosos autores se han dedicado a su estudio. Los termogramas de fusión obtenidos por calorimetria diferencial de barrido a partir de aceito de palma cristalizado rápidamente muestran dos transiciones endotérmicas una de bajo punto de fusión denominada liquido y otra de alto punto de fusión llamada sólido. En general, la mayoria de los autores llama oleina a la fracción de menor punto de fusión y estearina a la fracción de alto punto de fusión. Cuando el aceite de palma es enfriado muy rápidamente cristaliza en las formas a y $\beta$ simultáneamente. Deroanne (1975 a,b,a,d, 1976 a.b, 1977) hizo corresponder la forma a la fracción liquida y la $\beta$ a la solida. Este fonómeno llamado doble cristalización fue observado por este autor 
también en manteca con lo que concluyó que la doble cristalización ocurriria en todas las grasas que contuvieran dos tipos de cristales mixtos. Estos resultados son importantes desde el punto de vista tecnológico ya que el éxito del fraccionamiento de una grasa es función en primer lugar de la calidad de la filtración. Bsta sólo se puede realizar en la industria si los cristales son de la forma $\beta^{\prime}$. La relación entre el polimorfigmo, la morfologia y la filtración de los cristales debe ser conocida para poder poner a punto la tecnologia del fraccionamiento sin solvente de una grasa. Deroanne describió los cristales a como de estructura laxa pero dada su inestabilidad influyen poco en la filtración. Los cristales $\beta^{\prime}$ son fuertemente ramificados, forman esferulitas compactas que filtran muy bien. Los cristales $B$ son filamentosos y colman rápidamente el filtro (Deroanne, 1877).

Perron ( $1978 a, b, 1984$, 1986) estudiando aceite de palma por calorimetria diferencial de barrido observó que cuando este se fundia aparecian las dos transiciones endotérmicas mencionadas por Deroanne en sus trabajos. Por resonancia magnética nuclear determinó el contenido de solidos y lo relacionó con el diagrama calorimétrico. La primera transición endotérmica correspondia a una pequeña diferencia de variación en el contenido de sólido sin embargo era muy endotérmica. De acuerdo a la interpretación dada por el autor el fuerte intercambio calorico observado se deberia a la transformación de la forma a inestable en formas más estables. La segunda transición endotérmica en cambio correspondería a formas de mayor estabilidad.

Es bien conocido que el comportamiento térmico de una grasa es importante para su utilización. Sin embargo no se han ostable- 
cido correlaciones que permitan ligar este comportamiento a la composición en trigliceridos y poder prever asi para cualquier mezclas de grasa sus propiedades. Perron (1986) ha propuesto una primera aproximación a este problema basándose en la noción de triglicéridos inductores de cristalización y en 1 a influencia del numero de carbonos e insaturaciones en relación a las propiedades de las fases sólidas engendradas por estos triglicéridos. Ha sugerido un método para predecir el tenor de sólidos en función de la temperatura a partir de la composición en triglicéridos de la grasa considerada.

Habiamos mencionado que la forma polimórfica en que cristaliza un triglicérido depende fundamentalmente de dos factores: su naturaleza quimica y las variables de procesamiento. Respecto de la composición guimica es necesario citar la tendencia a la formación de cristales mixtos cuando se hallan presentes triglicéridos asimétricos que aumentan la intersolubilidad de los cristales (Chikamy, 1967). Los fenómenos son más simples cuando la cristalización es en presencia de solventes. Dada la mayor solubilidad de las moléculas los cambios cristalinos se harán preferencialmente dando las formas más estables. Cada forma polimórfica tiene un punto de transición que corresponde al punto de intersección de las curvas de energia libre de las diferentes formas cristalinas con la curva de energla libre de la sustancia liquida. En el caso de los triglicéridos la temperatura de fusión es función de la forma polimórfica y de la naturaleza de los triglicóridos (isomeria geométrica, de posición y composición en ácidos grasos).

En el caso de mezclas, es decir en la mayor parte de los casos prácticos, interviene además el grado de compatibilidad e 
incompatibilidad de los triglicéridos presentes. La naturaleza quimica de los aceites influye en la cristalización dado que esta depende de la distribución de los ácidos grasos sobre el glicerol, de la presencia de dobles ligaduras, de la configuración geométrica y de su longitud de cadenas. Los aceites de soja y girasol son B tendientes (Madsen, 1973); en estos aceites el go\% de los triglicéridos tienen cadenas de 18 carbonos; el aceite de algodón es $\beta^{\prime}$ tendiente (Merker, 1958), el contenido de acidos grasos de 16 carbonos en este aceite es superior al de girasol y soja (alrededor de 25\%) y el aceite de palma presenta el fenómeno de la doble cristalización de las grasas semisólidas (Deroanne, 1874, 1976, 1977). La presencia de mono y diglicéridos influye en el tipo de cristal que se obtiene.

En el fenómeno de cristalización tambien influye la naturaleza de la fase liquida presente. Por ejemplo si se considera la cristalización de triestearina en aceite de mani y en parafina se observa que en ambos casos se obtienen iguales cantidades de cristales pero que estos difieren en tamaño. Si se miden las superficies especificas de los cristales a $10^{\circ} \mathrm{C}$ y $40^{\circ} \mathrm{C}$ los cristales en aceite de mani resultan tener un valor doble del correspondiente a los cristales en parafina (Knoester y col., 1968).

Entre los factores tecnológicos que influyen en el polimorfismo podemos citar la velocidad de cristalización, la agitación, la curva o gradiente de enfriamiento, el solvente de cristalización y procesos de modificación industriales de las grasas.

Las diferentes formas cristalinas (sub $\alpha, a, \beta^{\prime}$ y $\beta$ ) pueden ser obtenidas dependiendo de la velocidad de enfriamiento. Un enfriamiento muy rápido provoca la aparición de las formas sub-a y a 
mientras que un enfriamiento más lento provoca en general la aparición de las formas $\beta$, y $\beta$ (Van Den Tempel, 1988). La forma a es inestable y cuando es posible un aporte de energia se transforma en $\beta^{\circ}$ y $\beta$. Estas transiciones se realizan mas fácilmente manteniendo los cristales a una temperatura ligeramente superior al punto de fusión de la forma polimórfica deseada.

En el caso del fraccionamiento de grasas la determinación de la velocidad de cristalización permite predecir una buena o mala separación de las diferentes fracciones. Como ejemplo consideraremos la manteca de nuez de palma refinada y la manteca de sebo refinada. En el caso de nuez de palma cuando se usa un programa rápido de temperatura se observan dos fracciones. S1 la velocidad es lenta se encuentra una sóla fracción. Esto muestra gue será dificil fraccionar la nuez de palma sin solvente. En el caso del sebo refinado la separación es neta aún con programas de temperatura lentos y el fraccionamiento sin solventes no traerá problemas.

La presencia de mono y diglicéridos modifica la velocidad de cristalización. Cuando el aceite de mani no posee mono y diglicéridos la velocidad de cristalización es más rápida que en presencia de $5 \%$ de monoglicéridos y esta velocidad se ve muy disminuida cuando el aceite contiene $5 \%$ de diglicéridos. Normalmente la concentración de diglicéridos es superior a la de monoglicéridos en los aceites no refinados.

La interesterificación puede influenciar la velocidad de cristalización aun siendo parcial. Un ejemplo clásico es la cristalización de las materias grasas después de haber sido desodorizadas. En el caso de la manteca de cacao donde la desodorización 
se hace a alta temperatura, la cristalización es tan mala que no puede ser utilizada en chocolateria. Cuando se sonete una nezcla de aceite de girasol no hidrogenado y aceite de girasol hidrogenado de punto de fusión $50^{\circ} \mathrm{C}$ a este tratamiento la separación neta de las diferentes fracciones que se encuentra antes de efectuar este procesamiento no se obtiene con posterioridad, es practicamente imposible diferenciar las fracciones inicialmente presentes ( Tafic y col., 1868). La velocidad de cristalización asi como la separación de las diferentes fracciones serán totalnente diferentes antes y después de la desodorización. Aún para una nateria grasa sin mezcla se obtiene una diferencia neta según la temperatura y la duración de la misma. En el caso del aceite de palma las diferencias de comportamiento son notables. La fracción de alto punto de fusión aumenta de manera sensible después de haber sido desodorizada. Aunque la interesterificación no sea conpleta al finalizar el proceso los cambios se manifiestan influyendo fuertemente sobre el fraccionamiento ulterior. Estos cambios que se pruducen al fraccionar no son reproducibles. La interesterificación durante la desodorización es catalizada por impurezas (tierras decolorantes, jabones) y esta cantidad de inpurezas varia de un proceso a otro con lo cual las cinéticas de las reacciones de interesterificación son muy diferentes.

Otro proceso industrial en el cual se producen modificaciones en las caracteristicas de cristalización es el templado. Este proceso consiste en colocar a temperatura entre $25^{\circ} \mathrm{C}$ y $35^{\circ} \mathrm{C}$ durante dos o tres dias margarinas o materias grasas (shortenings) y otros productos con el fin de nejorar sus propiedades de plasticidad, sus propiedades cremosas y el conportaniento en el horneado 
(Haighton, 1876). Shortenings mantenidos a $10^{\circ} \mathrm{C}, 23^{\circ} \mathrm{C}, 26^{\circ} \mathrm{C}$ y $30^{\circ} \mathrm{C}$ durante dos y nueve dias y posteriormente almacenados a $23^{\circ} \mathrm{C}$ presentaron transiciones $\beta^{\circ} \longrightarrow \beta$ como resultado de los tratamientos indicando que el templado influye en el incremento de la firmeza. Las muestras mantenidas a mayores temperaturas por mayor tiempo se endurecen más (Moziar y col., 1989).

\subsection{Cristalización de soluciones y aleaciones}

La solidificación de una solución salina de agua y cloruro sódico, no se efectúa generalmente a una temperatura única, como ocurre con el agua o con los metales puros, sino que se verifica entre determinados limites comenzando la cristalización a una cierta temperatura y terminando a otra inferior. Si ensayamos una serie de soluciones de concentración creciente, comenzando por el agua pura, y continuamos luego con soluciones cada vez más ricas en sal, vemos que la temperatura a la cual comienza la cristalización disminuye de solución en solución y existe una concentración llamada eutéctica que es la concentración correspondiente a la temperatura de solidificación inferior. Al continuar enriqueciendo en sal la solución se eleva la temperatura de comienzo de la solidificación. La solución que tiene la concentración eutéctica se solidifica brusca y completamente a una temperatura determinada, como sucede con el agua o cualquier sustancia pura y su curva presenta un sólo punto de detención a $-22^{\circ} \mathrm{C}$ para el eutéctico y $0^{\circ} \mathrm{C}$ para el agua. Las curvas de composición hipo e hipereutécticas presentan dos puntos de detención; el punto donde comienza la so- 
lidificación que es igual a $-10^{\circ} \mathrm{C},-10^{\circ} \mathrm{C}$ y $-8^{\circ} \mathrm{C}$ para soluciones con $10 \%, \quad 15 \%$ y $33 \% \mathrm{P} / \mathrm{V}$ de $\mathrm{C} 1 \mathrm{Na}$ respectivamente y un segundo punto igual para todas a $-22^{\circ} \mathrm{C}$. Las soluciones de concentración $10 x$ y $15 \%$ de ClNa son mas diluidas que la concentración eutectica $(23,5 \%)$. En ambas en el primer punto de detención precipita una cierta cantidad de cristales de hielo. Al ir disminuyendo la temperatura se solidifica más agua y la concentración del liquido residual sigue aumentando hasta llegar a la del eutéctico en donde se solidifica bruscamente todo el liguido. Cuando se enfria una solución de concentración superior a $23,5 \%$ de ClNa los cristales que precipitan son de ClNa. Al descender la temperatura continúa solidificando hasta llegar a $23,5 \%$ y se solidifica todo de una vez.

Eenómenos similares a los descriptos ocurren en las aleaciones de metales. Dichas aleaciones son totalmente solubles en estado liquido peróen el estado sólido se presentan tres casos: insolubilidad como en el caso de bismuto y cadmio, solubilidad parcial como en el de cobre y plata y solubilidad total como las aleaciones de oro y plata. Las aleaciones de bismuto y cadmio se comportan de manera similar a las soluciones salinas. Las aleaciones de cobre y plata cuando solidifican precipitan cristales que no son puros ni de cobre ni de plata sino que contienen un porcentaje de ambos. Los cristales que van precipitando son de composición diferente $y$ existe entre ellos bastante heterogeneidad. Si el enfriamiento es lento la composicin de cristales vecinos se homogeiniza. Las aleaciones de oro y plata después de solidificar tienen el mismo grado de homogeneidad que cuando estaban fundidas y todos los cristales son de la misma composición o sea que en esta- 
do sólido, ambos metales son totalmente solubles uno en otro. Solidifican a temperaturas inferiores al oro y superiores a la plata. La solidificación no es brusca sino que la precipitación de cristales ocurre en un rango de temperaturas. En el caso de que la velocidad de enfriamiento sea relativamente rápida, los fenómenos se producen en forma algo diferente, la difusión es imperfecta y los granos formados por la agrupación de cristales de diferente composición están constituidos por capas concéntricas heterogéneas cuyas composiciones varian del centro a la periferia (Apraiz Barreiro, 1964).

\subsection{Cristalización de grasas y aceites}

En el curso de la cristalización de las grasas aungue cada triglicérido presente en la mezcla tiene una forma cristalina preferencial, los mismos no cristalizan en forma independiento sino que varios de ellos de diferente punto de fusión forman una red cristalina común. Los trigliceridos en base al grado de saturación pueden dividirse en los siguientes grupos: triglicéridos trisaturados que juegan un papel importanto en la formación de la estructura sólida, los disaturados que participan en la formación de la estructura y en el deslizamiento y el resto que son encontrados en la fase de grasa continua participando en el deslizamiento. Los cristales se hallan rodeados de grasa liquida. La posibilidad de deslizarse depende de la solidez de la estructura (Chikámy, 1965).

En general las grasas vegetales y en especial aquellas que 
contienen en su mayoria ácidos gragos de idéntico número de carbonos tienden a presentar un mayor polimorfismo que las grasas animales. La transesterificación varia mucho las caracteristicas polimóricas y aumonta la asimetria do los triglicoridus. Los triglicéridos además del carácter polimorfo son de caracter monotropo o sea que las transformaciones de una forma cristalina a otra ocurren en la dirección de las formas más estables, es decir la transformación se produce en el sentido $\alpha \longrightarrow \beta^{\circ} \rightarrow \beta$. La transformación $a \longrightarrow \beta^{\prime}$ es rápida mientras que la $\beta^{\circ} \longrightarrow \beta$ es lenta por 10 tanto este fenómeno no influye tanto en 1 a producción sino durante el almacenamiento y a menudo va acompañado de textura desagradable (arenosa al tacto).

Si bien las grasas y aceites son mezclas complejas de especies quimicas pueden analizarse en diagramas de dos componentes como sistemas binarios o ternarios tomando los componentes mayoritarios como rectores del comportamiento de la mezcla. Las variaciones de composición de una mezcla repercuten proporcionalmente sobre sus componentes. Los triglicéridos presentan un comportamiento térmico muy complicado; los fenómenos explicados para aleaciones y soluciones salinas se presentan todos simultaneamente. Se encuentran tres tipos de sistemas dependiendo del par de triglicéridos rectores del comportamiento de la mezcla: solución sólida continua, eutéctico y pseudocompuestos.

Estas interacciones pueden diferenciarse mediante el conocimiento de la relación sólido liquido de la grasa en cuestión. Para ello pueden ser empleados diversos métodos: dilatación, microcalorimetria dinámica y espectroscopia de resonancia magnética nuclear. La dilatación se basa en la diferencia entre el volumen 
especifico de las grasas en estado liquido y sólido. Con los datos de los volumeries especificos se construye un diagrama temperatura en función de los porcentajes de oada componente para cada relación sólido liquido (Chikámy, 1966). Los diagramas do isodilatación ilustran bien el comportamiento de fusión de las mezclas de materias grasas (Bailey, 1950). Estos diagramas se pueden construir de manera ańloga a partir de los datos obtenidos con los otros métodos mencionados.

En un sistema en el cual los componentes forman solución sólida continua es decir cuando la compatibilidad es total entre los dos componentes cualquiera sean las proporciones de la mezcla considerada no se ven variaciones importantes en los puntos de fusión. Es el caso de SSS/ESS; POS/SOS y POP/POS. Un eutéctico presenta un punto de fusión minimo para una concentración dada lo que se traduce en una dilatación minima. Para citar ejemplos, forman eutécticos los pares PPP/SSS; EEE/SOS; PPP/LLL y SEE/SOS. La formación de pseudocompuestos se manifiesta por el contrario por la aparición de un punto de fusión y de dilatación máximos. No se trata de formación de compuestos de naturaleza quimica sino de un arreglo cristalino preferencial; es el caso de SSO/SOS y POP/OPO. Triglicéridos parecidos no son necesariamente compatibles asi hay una compatibilidad mayor entre PPP/SOS que entro PPP/POP y el conportamiento es corrientemente imprevisible. Por ejemplo: se obtiene una fracción sólida por la simple mezcla a temperatura ambiente de fracciones liquidas de tocino y aceite de palma. Se trata de un pseudocompuesto que no se forma en los componentes tomados individualmente. La compatibilidad, la incompatibilidad y la existencia de los diversos sistemas están ligadas al polimorfismo 
de los cristales y a la orientación de las cadenas gliceridicas en la red cristalina. Las distintas configuraciones estereoguimicas de la molécula en el cristal con diferentes orientaciones del arreglo en zig zag de las cadenas gliceridicas conducen a las formas cristalinas más o menos compactas ya descriptas. La configuración menos compacta es la a y la más compacta es la $\beta$. Las formas $\beta^{\prime}$ son intermedias. Se admite actualmente que salvo en el caso de triglicéridos de cadena corta ( $C: 12, C: 14$ ) un enfriamiento rápido produce un estado vidrioso y que aportando energia calorica pasa por las siguientes etapas: $\gamma \rightarrow a \rightarrow \beta^{\prime} \rightarrow \beta$. La energia necesaria para estas transformaciones depende de la estructura cristalina. Asi la transición de una forma cristalina a otra es relativamente rápida en el caso de las cadenas con número par de carbonos y de los glicéridos simétricos y lenta en el caso de las cadenas con número impar de carbonos y de los triglicéridos asimétricos (Chikámy, 1966). Evidentemente la velocidad de transición disminuye con el aumento de la longitud de la cadena. En los diagramas obtenidos por rayos $X$ de los triglicéridos los espaciamientos largos son función de la longitud de las cadenas de los acidos grasos y del ángulo de inclinación con respecto al plano de los grupos metilos. Lutton (1967, 1972) ha propuesto una representación en si11 para los triglicéridos en concordancia con los resultados hallados por Rossel (1973) y por Knoester y colaboradores (1872) y han sido confirmados por medida de los cristales de triglicéridos saturados de largas cadenas. Existen diferentes arreglos posibles de las moléculas de triglicéridos en el cristal. Se encuentran más corrientemente las estructuras en doble o en triple cadena. Si los ácidos grasos solo difieren en longitud de cadena en dos carbonos 
se encuentra una estructura de doble cadena donde los trigliceridos se disponen cabeza cola sea verticalmente (forma a y ciertas formıs. $\beta^{\prime}$ ) o con un ángulo de inclinación más marcado $\left(\beta^{\circ}\right.$ y $\beta$ ). Si los ácidos difieren en longitud de al menos cuatro carbonos los espaciamientos largos aumentan generalmente para formar una estructura en triple cadena. Esta se produce también cuando esta presente un ácido graso insaturado. En el caso de triglicéridos con largos de cadena muy diferentes tales como los acetoglicéridos la estructura es de simple cadena (Simpson y Hagemann, 1975).

En triglicéridos el pasaje de una forma a otra es a veces lento porque las formas cristalinas pueden corresponder a arreglos moleculares muy diferentes. Es asi que los triglicéridos SOP en la manteca de cacao pasan muy lentamente de una estructura del tipo doble cadena a una estructura del tipo triple cadena lo gue podria explicar los periodos de revenido prolongados que son necesarios para esperar la forma estable de los cristales do manteca de cacao (Paulicka, 1973) y de la fracción intermediaria del aceite de palma, rica en POP y POS. Las velocidades de transición dependen en parte de las longitudes de las cadenas y por supuesto de la temperatura. Los fenómenos se complican por la interacoión de los ácidos grasos libres y glicéridos parciales como los diglicéridos que se producen en el fraccionamiento del aoeite de palma. Estos dan lugar a la formación de eutécticos y por otra parte prolongan la duración de la vida de las formas inestables (Persmark y colaboradores, 1974; Riiner, 1971; Jacobsberk, 1876). 


\section{HODIFICACION DE GRASAS Y ACEITES}

El aceite puede obtenerse de diversas especies vegetalos. A partir de leguminosas como por ejemplo la soja o de oleaginosas como girasol y mani y otras menos frecuentes en nuestro pais como tung, nabo y colza. A su vez puede provenir de diferentes partes del vegetal como frutos por ejemplo el aceite de oliva o semilla como girasol, algodón, soja, etc. Los distintos aceites se procesan en forma diferente según su origen. Pocos pueden ser consumidos tal como se extraen. La mayoria de los aceites crudos contienen apreciables cantidades de impurezas, tienen con frecuencia colores oscuros, son turbios y poseen olores desagradables. Por lo tanto deben ser sometidos a una serie de transformaciones para ser utilizados en productos manufacturados o como aceites de cocina.

En el caso del aceite de girasol el contenido de aceite extraido por prensado o extracción con solvente de las semillas depende fundamentalmente del tamaño de particula resultante de la molienda. Esto se ve influenciado por la homogeneidad de la masa molida de la que se extrae. Ensayando diversos métodos de molienda Kochhar y Rossell (1987) llegaron a la conclusión que para optimizar la extracción la mezcla debe tener un tamaño de particula no mayor de dos milimetros y menos del $12 \%$ de las particulas no mayores que $1,18 \mathrm{~mm}$.

Las etapas de refinación para aceite de girasol, tomado a modo de ejemplo se pueden esquematizar de la siguiente manera: Extracción- desgomado- neutralización- lavado con agua- secadoblanqueado-filtración- desodorización- segunda filtración. 
El objetivo de cada una de estas etapas es el siguiente:

Desgomado: permite la remoción de fosfolípidos, azúcares, resinas, compuestos proteicos, trazas de metal, etc.

Neutralización: posibilita la eliminación de ácidos grasos, pigmentos, fosfolipidos, compuestos sulfurados, compuestos insolubles en aceites y solubles en agua.

Lavado: mediante este procedimiento se separan los jabones.

Secado: permite la remoción del agua.

Blanqueado: posibilita la eliminación de pigmentos, productos de oxidación, trazas de metales y jabones.

Filtración: permite la separación de las tierras de blanqueo y los insolubles.

Desodorización: mediante este procedimiento se eliminan sustancias volátiles como ácidos grasos libres, mono y diglicéridos, aldeh1dos y cetonas, hidrocarburos, compuestos sulfurados y productos de descomposición de pigmentos que le confieren al aceite un olor desagradable.

Segunda filtración: se realiza antes de almacenar, con filtros que retienen particulas de entre 30 a 10 micrones (Hamilton y Bhati, 1980). 
El aceite de girasol que se usa para cocina sufre estos procesos de refinación y se obtiene un liquido entro pálido y amarillo brillante. Además es sometido a un proceso que no es común a todos los aceites: la winterizacion. Este proceso consiste en cristalizar las ceras a baja temperatura las que luego son removidas por filtración. Esto hace que al colocarlo en el refrigerador a $5^{\circ} \mathrm{C}$ el aceite permanezca limpido, sin turbiedad. Por esta caracteristica son ideales aceites de mesa (Drachmann, 1932; Jamieson, 1942 ; Eckey, 1954).

Cuando la tecnologia estaba poco avanzada se utilizaban materias grasas sin modificar para usos determinados por ejemplo manteca para untar el pan, aceite de oliva para la preparación de mayonesas, manteca de cacao para la fabricacion de chocolate, etc. La utilización de grasas y aceites estaba limitada por la producción y los precios fluctuaban en consecuencia. La mezcla de los cuerpos grasos ha permitido la elaboración de productos aprobados por el consumidor $y$ que poseen una gran versatilidad en cuanto a su formulación lo que representa una enorme ventaja económica para la industria. Un ejemplo de esto es la margarina (Jacobsberg, 1976; Jacgmain, 1977).

Para obtener cuerpos grasos con caracteristicas diferentes a las de las materias primas pueden ser aplicados tres métodos: hidrogenación, interesterificación y fraccionamiento.

\subsection{Hidrogenación}

En 1902 se patentó un método do hidrogenación de grasas y 
ácidos grasos en fase liquida usando niquel como catalizador lo que amplió las posibilidades de empleo de las materias grasas (Norman, Patente DP 141029, 1902). Desde ese entonces el proceso se utiliza en la industria para transformar aceites insaturados liquidos en materias grasas más saturadas. En esta operación el punto de fusión del producto final se ve incrementado respecto del punto de fusión inicial, junto con el punto de fusión la estabilidad de grasas y aceites se ve aumentada.

Para llevar a cabo este proceso es necesario dispersar hidrogeno en aceite en presencia de un catalizador. El aceito usado es generalmente pretratado, esto es, neutralizado y blangueado dado que debe estar limpio y seco. El nivel de ácidos grasos libres no debe ser mayor que $0.15 \%$ y debe estar libre de otros venenos del catalizador como gomas, jabones y sulfuros. El hidrógeno debe tener una pureza del orden de $99,5 \%$. El catalizador más empleado es niquel con un soporte de tierras de diatomeas y generalmente el contenido de niquel empleado es del $20 \%$.

Las funciones del catalizador son fundamentalmente modificar la velocidad de reacción y dirigir la reacción en forma especifica. La catálisis puede ser de tipo homogeneo o heterogeneo. En la catálisis homogénea ambos reactivos se hallan en la misma fase, en estado gaseoso o lo que es más usal en materias grasas en solución. Este tipo de catálisis es de poca importancia. En catálisis heterogenea los reactantes y el catalizador pueden encontrarse en dos o tres fases. La mayoria de las reacciones de hidrogenación son de la siguiente clase: el catalizador es sólido, el hidrógeno gaseoso y la sustancia a hidrogenar es un liquido.

Lo más destacable de este proceso es la selectividad del ca- 
talizador es decir este es capaz de distinguir entre los diferentes ácidos grasos. En primer lugar el catalizador debe atacar lus ácidos grasos más insaturados y luego otros más saturados hasta alcanzar el grado de insaturación deseado. La hidrogenación total no es un caso frecuente. La selectividad es muy importante desde el punto de vista nutricional; el ácido linoleico es un ácido graso esencial para los animales y debe estar presente en la alimentación. Un ejemplo en el cual la importancia de esta selectividad se ve manifiesta es la hidrogenación de aceites de pescado proceso en el que se saturan selectivamente los ácidos clupanodonicos, araquidónico y linolénico produciéndose oleico y linoleico, dando como resultado un producto final de alto valor nutritivo.

El cobre tiene una selectividad apreciablemente mayor que el niquel pero no es empleado por ser un poderoso pro-oxidante y por la dificultad de su remoción luego de finalizada la reacción; además su actividad y propiedades al ser reempleado son muy inferiores a las del niquel.

Para conocer las condiciones en las cuales debe proceder la reacción, en particular la temperatura y la presión óptimas de operación, es indispensable un estudio termodinámico. Sin catalizador la hidrogenación procede a velocidad lenta; sólo pocas moléculas de sustancia reactiva tienen niveles de energia suficientemente altos para reaccionar. El efecto del catalizador es la disminución de la energia de activación. Si la energia de activación es conocida y también la concentración de reactivos puede calcularse la constante de equilibrio. Dado que su valor depende de la temperatura se puede determinar las condiciones optinas de reacción. Para la mayoria de las reacciones de hidrogenación las 
bajas temperaturas son termodinámicanente favorables. Si la temperatura es elevada la hidrogenación aún es posible si la presión de hidrógeno es suficientemente alta. En muchos casos un incremento relativamente bajo de la presión impide la hidrogenación. Sin embargo el aumento de presión tiene en otros casos un efecto cinético: se incrementa la velocidad de reacción. Dado que la hidrogenación es una reacción exotérmica en operaciones prácticas debe tenerse cuidado proveyendo un buen enfriamiento. El calor de hidrogenación de las grasas es de $25 \mathrm{Kcal} / \mathrm{mol} / \mathrm{doble}$ ligadura que se satura entre $130^{\circ} \mathrm{C}$ y $200^{\circ} \mathrm{C}$.

El mecanismo de reacción propuesto es el siguiente: durante la absorción de hidrógeno se forma probablemente un complejo $\pi$ entre el centro reactivo del catalizador y la olefina. Es interesante destacar que pocos elementos actuan como catalizadores en la hidrogenación ( $\mathrm{Ee}, \mathrm{Co}, \mathrm{Ni}, \mathrm{Cu} \mathrm{y}$ los metales nobles). Todos ellos tienen distancias entre metales de $2,47 \mathrm{~A}-2,54 \mathrm{~A}$ o 2,70A-2,80A. La responsable de la formación de la ligadura metal-olefina es la donación de electrones en orbitales $\pi$ de la olefina a orbitales aceptores vacantes tipo o del átomo de niquel en la superficie del catalizador $y$ una unión tipo retrodonación de los orbitales dxy o de los orbitales hibridos $d \pi-p \pi$ del niquel a orbitales aceptores $\pi^{*}$ de la olefina. Esto involucra un carácter de doble unión en la unión metal olefina. La olefina que forma parte del complejo es hidrogenada y luego desadsorbida con lo cual el nivel energético decrece y es fácil retener un atomo de hidrógeno. La hidrogenación del complejo olefina no es absolutamente necesaria, si el $\mathrm{Ha}$ no es suficiente la olefina se separa del catalizador sin hidrogenar. En este caso la isomerización tiene lugar en cierta extensión. Los 
ácidos grasos insaturados cis durante el proceso son transformados en derivados trans de mayor punto de fusión que no se hallan en los aceites naturales en cantidades significativas. Los hidrogenados producidos por catálisis a partir de aceites son luego usados para la preparación de productos manufacturados tales cono margarinas y shortenings (Zschau, 1976).

\subsection{Interesterificación}

La interesterificación es un proceso de menor importancia industrial que la hidrogenación. Como su nombra lo indica consiste en obtener ésteres diferentes a los iniciales. En la industria de grasas y aceites este proceso se usa para producir mezclas de grasas de distintos origenes que posean caracteristicas diferentes a las grasas originales. Tambien se emplea para enriquecer mezclas grasas en un ácido graso en particular.

Los catalizadores más empleados en la actualidad para interesterificar grasas son los siguientes:

a) Metóxido o etóxido de sodio a $80^{\circ} \mathrm{C}-100^{\circ} \mathrm{C}$.

b) Sodio metálico a $100^{\circ} \mathrm{C}-120^{\circ} \mathrm{C}$.

c) Sodio/Potasio $22 / 78 \% \mathrm{P} / \mathrm{P}$ de punto de fusión $-12,5^{\circ} \mathrm{C}$ a temperaturas alrededor de cero grado.

d) Butóxido de Potasio.

Este proceso se aplica tanto a aceites como a sus mezclas siendo posible predecir la composición en triglicéridos resultante conociendo la composición en acidos grasos del aceite o 
la mezcla de partida. Se conocen dos formas de efectuar esta reacción: en una fase llamada "al azar" y en dos fases denominada interesterificación dirigida. La reacción dirigida es un caso especial de la reacción "al azar" en la cual mediante la disminución de la temperatura de la mezcla de reacción se consigue la cristalización de los trigliceridos saturados modificando el equilibrio de la fase liquida causando la formación de una fracción de más alto punto de fusión. De esta manera por ejemplo el aceite de algodón que contiene $28 \%$ de ácidos grasos saturados pero gue es liquido a temperatura ambiente en su estado natural puede transformarse en sólido a dicha temperatura. Para que la reacción sea completa es necesario comenzar con un aceite seco, con un contenido de ácidos grasos preferentemente inferior a $0,05 \%$ y libre de peróxidos dado que ellos reaccionan con el catalizador. El tiempo de reacción cuando se trabaja en vacio es de 30 min al final del cual se detiene la catálisis por adición de agua, soda caústica, ácido diluido o $\mathrm{CO}_{2}$. La mayoria de las plantas usadas son piloto y su uso más común es modificar en batch las caracteristicas de neutralizantes y blanqueadores. Sin embargo se han construido unidades de uso semicontinuo y continuo (Von Speisefetten, 1973).

\subsection{Fraccionaniento}

El fraccionamiento permite obtener aceites y fracciones sólidas en las cuales las propiedades fisicas son netamente diferentes de las del aceite original.

Esta técnica ha sido muy empleada en el estudio de diversos 
sistemas y para usos industriales. La posibilidad de obtener una separacion neta entre las fracciones sblidas y liquidas de una mezcla grasa depende fundamentalmente del método de enfriamiento, el cual induce la forma y tamaño de los cristales de grasa obtenidos (Kreulen, 1976). El enfriamiento rápido causa gran sobresaturación y origina una enorme cantidad de cristales pequeños amorfos con pobres propiedades de filtrado. Esta forma microcristalina se transforma en la forma metaestable a gue presenta las mismas caracteristicas de cristalización y tendencia a desarrollar cristales mixtos. Un enfriamiento gradual de un aceite produce formas $\beta$ y $\beta^{\prime}$, macrocristalinas que pueden ser separadas fácilmente de la fase liquida. La cinética de cristalización de grasas y aceites puede ser descripta por ecuaciones frecuentemente usadas para sistemas inorgánicos (Van Putte y Bakker, 1987).

Las propiedades quimicas y fisicas de las fracciones separadas dependen de las condiciones de fraccionamiento y del destino dado a la grasa. Las propiedades de las fracciones pueden modificarse por hidrogenación, interesterificación y mezclas o combinaciones de estas técnicas. El fraccionamiento se ha aplicado a muchos sistemas complejos. Los ejemplos que figuran a continuación fueron seleccionados por el avance que representaron en el campo de las grasas y aceites y por su relación con el estudio realizado por nosotros.

Se han desarrollado técnicas de fraccionamiento de aceites vegetales, animales y de pescado, y en particular de aceite de palma en presencia de mezclas binarias de solventes que se basan en las diferencias de solubilidad de la fracción estearina y oleina las cuales se separan por centrifugación (Ong, 1883). Amer 
(1985) ha estudiado el fraccionamiento de mantecas fabricadas en invierno $y$ en verano. Básicamente el proceso consiste en un enfriamiento lento $y$ controlado $y$ una pequeña estabilización a la temperatura de fracionamiento con posterior separacion de los cristales de la fase liquida por filtración al vacio. Las fracciones fueron caracterizadas por sus acidos grasos, trigliceridos y procesos de fusion y cristalizacion. Los reaultados obtenidos mostraron que las fracciones sólidas revelaban en sus termogramas la presencia de un contenido importante de liquidos y que existian pequeñas diferencias entre las composiciones quimicas de las fracciones entre si. Arul y colaboradores (1888) fraccionaron grasa anhidra de leche por destilación molecular obteniendo dos fracciones liquidas, una semisólida y una sólida a temperatura ambiente. Las fracciones fueron caracterizadss con perfiles de fusión, indice de grasa sólida y composición en ácidos grasos y triglicéridos. Encontraron gue la temperatura del pico que representa la fusión se incrementaba progresivamente desde $8,8^{\circ} \mathrm{C}$ a $38,7^{\circ} \mathrm{C}$ de las fracciones liquidas a las sólidas. El contenido de grasa sólida resultó de $27,5 \%$ a $20^{\circ} \mathrm{C}$ en la fracción sólida mientras que en la leche inicial fue de $15,4 \%$. Los triglicéridos de cadena corta ( $\left.\mathrm{C}_{24}-\mathrm{C}_{34}\right)$ se concentraron en las fracciones liquidas, los de cadena intermedia ( $\mathrm{C}_{38}-\mathrm{C}_{40}$ ) en la fracción semisólida y los de cadena larga (Caz- $\mathrm{Cs} 4$ ) en la fracción sólida. Los acidos grasos de cadena corta ( $\left.\mathrm{C}_{4}-\mathrm{Ca}\right)$ gradualmente decrecen de liquido a solido. También se han realizado experiencias con aceite de fritado comercial (Sebedio y col., 1986). En este caso se emplearon dos métodos de fraccionamiento: el método estandarizado DGF-IUPAC-AOAC y empleando cartuchos rellenos. Se deseaba encontrar un método más 
rápido y económico que el método IUPAC para evaluar el deterioro de los aceites comerciales al ser usados para freir. Los resultados obtenidos con cartuchos rellenos (sep-pak) y por los metodos estandarizados fueron similares demostrando que estos últimos podian ser reemplazados por el primero. A efectos de obtener ácido - Y linolénico (cis 6,9,12-octadecatrienoico) a partir de aceite de grosellas se han ensayado diferentes posibilidades (Traitter y col., 1988). El fraccionamiento con úrea en metanol díb buenos resultados para separar a y $\gamma$ linolénico pero el ácido estearidónico no pudo ser separado con esta técnica. Mediante HPLC semiindustrial preparativa se obtuvieron fracciones de acido linolenico de $95 \%$ de pureza. En el caso de aceite de palma se ensayaron distintos métodos de fraccionamiento: rápido sin solvente, lento sin solvente $y$ mediante el empleo de detergentes. Las tres formas de procesamiento permiten obtener productos de diferente calidad, empleados con diferentes fines y con diferentes costos operativos (Deffense, 1985). Dimick y Manning (1987) estudiaron el fraccionamiento de manteca de cacao por cristalización en condiciones estáticas a distintas temperaturas: 26,$0 ; 28,0 ; 30,0 ; 32,0$ y $33,0^{\circ} \mathrm{C}$. Encontraron que para cada temperatura los cristales formados diferian en su composición en triglicéridos. Por ejemplo los cristales formados a $26,0^{\circ} \mathrm{C}$ tienen un incremento significativo en SOS $y$ disminución significativa en POP comparado con la manteca original. El porcentaje de SOS se incrementa de $26,0^{\circ} \mathrm{C}$ a $32,0^{\circ} \mathrm{C}$.

De todo 10 expuesto surge que el fraccionamiento sin solvente presenta ventajas respecto de otros métodos de fraccionamiento. Es el mas barato, fácil de llevar a cabo pero desgraciadamente el menos selectivo y limitado a ciertos productos simples como el 
aceite de palma, grasa de vaca y ciertos aceites hidrogenados. La retención de la fase liquida en la fase solida después de la filtración es siempre superior a la de otros métodos de fraccionamiento (Hinnekens, 1976). Existen dos clases de equipos comunmente empleados para este tipo de fraccionamiento: los que se emplean en procesos discontinuos como por ejemplo un sistena de cubas con doble pared o con serpentines, o una cuba de cristal1zación clásica con bomba de agitación y los utilizados en la cristalización en continuo entre los que se encuentran los cristalizadores cilindricos horizontales. El sistema que consiste en una cuba de cristalización clásica agitada por una bomba tiene el inconveniente de que la circulación de cristales que se provoca con la bomba frecuentemente conduce a una mala filtración. En el caso de los cristalizadores cilindricos horizontales, los factores más importantes a tener en consideración son la agitación y la relación superficie volumen de enfriamiento. La cristalización de las materias grasas exige tiempos de residencia suficientemente largos y una débil agitación. El inconveniente mayor en estos sistemas es la cristalización en las paredes. El rascado do los cristales provoca una turbulencia de los mismos que hace diflcil e 1 filtrado.

La programación de temperaturas utilizada en el fraccionamiento de una grasa depende del tipo do materia prima y del sistema de programación de temperatura. Hay diforentes métodos empleados para regular el enfriamiento: en el caso de fraccionamiento con un sistema "tirtiaux" la programación de temperatura se logra con una diferencia constante entre la solución a cristalizar y el liquido de enfriamiento. Otro sistema consiste en emplear una 
programación de temperatura lineal hasta el momento de la nucleación. El crecimiento de los cristales es provocado por un enfriamiento lento hasta el fin de la primera criatalización (Jaroslov, $1971)$.

En el caso de fraccionamiento sin solventes se obtiene siempre una mezcla de formas cristalinas. 


\section{OBJ ET I VOS}

El objetivo de este trabajo es estudiar el fraccionamiento de aceite de girasol hidrogenado por cristalizacion. Asi mismo se desea determinar las relaciones existentes entre las caracteristicas de cristalización de cada fracción y su composición quimica y establecer su posible aplicación a nivel industrial. 
Materiales

y

Me'todos 


\section{HUESTRA BHPLEADA}

La experiencia de fraccionamiento se efectuó por duplicado. En ambos casos la muestra utilizada fue provista por Molinos Rio de La Plata S.A.. La misma estaba compuesta por aceite de girasol hidrogenado llamado girasol $35^{\circ} \mathrm{C}$ utilizado para la preparación de la fase grasa de margarinas comerciales. En la primer experiencia se cargaron al tanque del equipo fraccionador $50 \mathrm{Kg}$ de aceite hidrogenado de indice de iodo $65 \mathrm{y}$ punto de goteo $35,5^{\circ} \mathrm{C}$ y en la segunda experiencia $27 \mathrm{Kg}$ de aceite hidrogenado de Indice de iodo 68 y punto de goteo $35,4^{\circ} \mathrm{C}$.

\section{FRACCIONAMIENTO}

\subsection{Equipo enpleado}

El equipo utilizado se construyó en nuestro laboratorio. Sirvió de inspiración fara el mismo una prensa diseñada por Haighton que fue empleada por Van der Hook (1969) para estudiar la estabilidad estructural de margarina. El esquema del equipo se muestra en la Figura 1. La bomba succiona el fluido del tangue y este pasa luego por un filtro MP 40-1 con malla de acero inoxidable. El fluldo penetra a la carcaza del filtro, atraviesa el mallado donde quedan retenidos los sólidos y regresa al tangue por un caño conectado al interior del filtro. Los liquidos son asi devueltos al tanque. El aceite hidrogenado do girasol que se frac- 
cionó circuló durante doce horas en un circuito cerrado a cada una de las temperaturas de eraccionamiento. Para mantener la temperatura constante se utilizó un criostato en el cual se hallaba contenido el tanque; en su tapa se coloco una resistencia ( $2 \mathrm{KW}$ ) y un termómetro de contacto (Frovi FW-21) conectados a un controlador de temperatura (CIDCA Modelo BC 07). Como liquido refrigerante se empleb agua enfriada por un serpentín por el que circulaba freón. La temperatura del agua se mantuvo a $2^{\circ} \mathrm{C}$ es decir $10^{\circ} \mathrm{C}$ por debajo de la última temperatura a la que se fraccionó el aceite hidrogenado. El controlador encendia y apagaba la resistencia según la temperatura subiera o bajara con una sensibilidad de medio grado centigrado. La agitación obtenida dada por la potencia de la bomba y los agitadores hizo que los equilibrios establecidos entre sólidos y liquidos fueran dinámicos. 


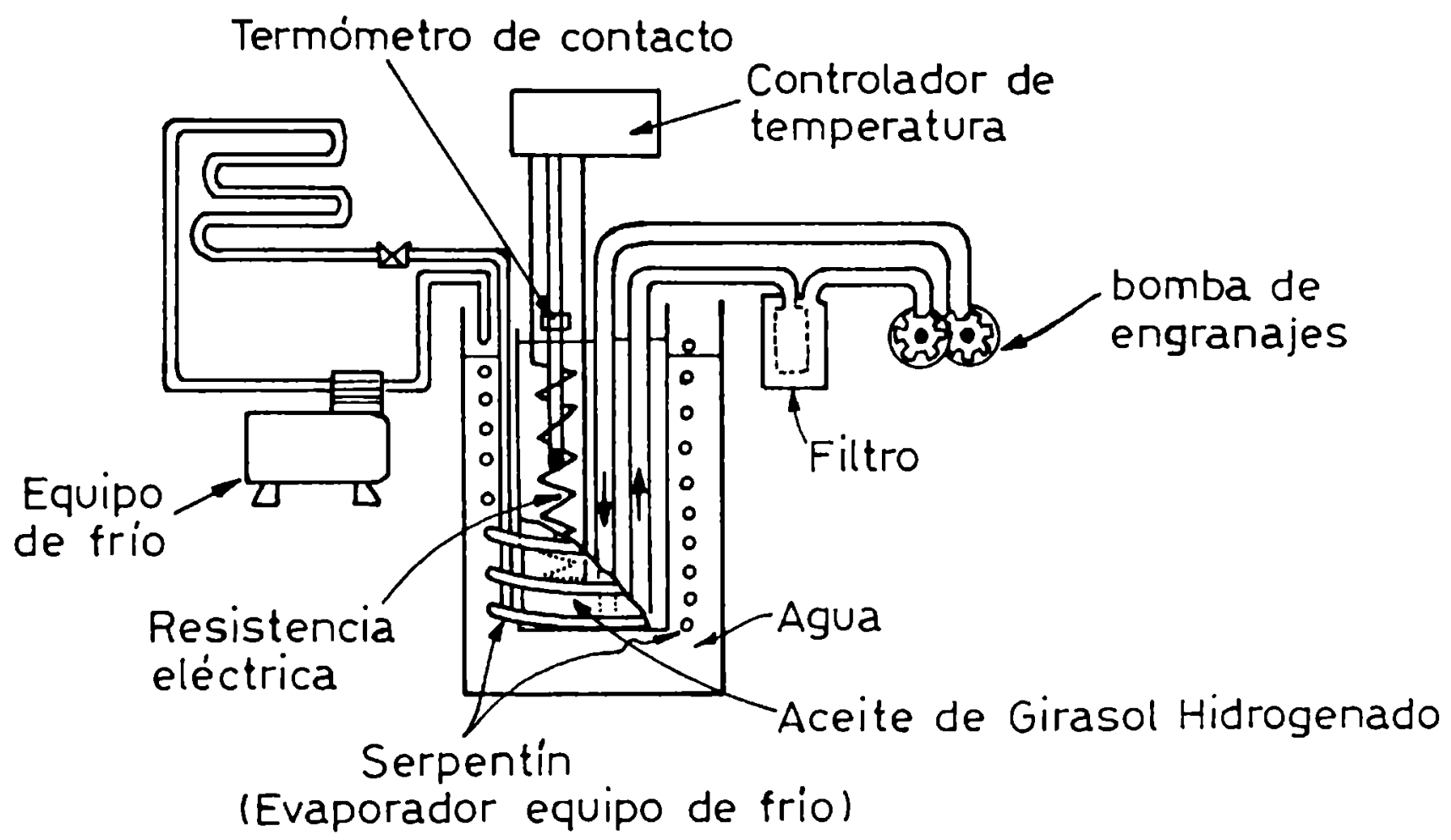

Figura 1. Equipo enpleado en el fraccionaniento de aceite de girasol hidrogenado 


\subsection{Método utilizado}

El fraccionamiento se comenzó en la primer experiencia a $41^{\circ} \mathrm{C}$ $y$ en la segunda a $38^{\circ} \mathrm{C}$. El aceite hidrogenado se fundió on el tangue a $45^{\circ} \mathrm{C}$ en forma controlada para evitar en lo posible su oxidación. Luego se hizo circular durante media hora y se descendió la temperatura hasta alcanzar la temperatura de inicio de la experiencia, se dejó cristalizar y se extrajo la totalidad del sólido acumulado en el filtro y una porción representativa del liquido del tanque. Después se bajó 1 a temperatura en $2^{\circ} \mathrm{C}$ en ambos casos y se procedió de igual manera siguiendo escalones de $2^{\circ} \mathrm{C}$ hasta llegar a una temperatura de $19^{\circ} \mathrm{C}$ en la primer experiencia y $12^{\circ} \mathrm{C}$ en 18. segunda. Las muestras fraccionadas a $3^{\circ} \mathrm{C}$ y $7^{\circ} \mathrm{C}$ correspondientes a la primer experiencia se obtuvieron en forma estática en un baño Haake F3 colocando el remanente del tangue en un cilindro cribado que permitia escurrir el liguido. Primero se obtuvo la fracción de $7^{\circ} \mathrm{C}$ y el liquido se volvió a fraccionar a $3^{\circ} \mathrm{C}$. Estas fracciones se estudiaron en cuanto a composición guimica (HPLC, GLC, RHN y IR) y estructura cristalina (DSC y Rayos X).

\subsection{Prueba piloto}

El equipo luego de haber sido conetruldo fue probado con aceite de girasol hidrogenado. A lo largo de las pruebas el diseño debió ser optimizado con el objeto de conseguir un mejor fraccionamiento. El equipo original constaba de una bomba centrifuga. En este tipo de bomba el aceite cristalizaba en su interior interrum- 
piendo el proceso. Debia desarmarse para limpiar y de esa manera la cristalización no correspondia al fin buscado, es decir que fuera muy gradual y que cada fracción tuviera una historia térmica similar a la anterior. Además presentaba el problema de tener que fundir cada vez al comenzar toda la masa del aceite hidrogenado con el consiguiente deterioro de la muestra. Esta bomba fue canbiada por una bomba de engranajes de 1 HP y 1 " de salida y entrada. La potencia de esta bomba obligó a cambiar los caños por otros de diámetro superior ( $\left.3 / 4^{\prime \prime}\right)$ que permitieron un mayor flujo. La posición del filtro en el circuito debió ser modificada. En un principio estaba colocado después de la bomba. En esta posición la temperatura en el filtro era superior a la del tanque. El filtro fue colocado antes de la bomba e inmediatamente a la salida del tanque. Al no pasar el fluido por la bomba antes de ser filtrado, la temperatura pudo ser controlada en los valores deseados.

Se habia pensado que el equipo funcionara dentro de una camara a $0^{\circ} \mathrm{C}$. El sensor de temperatura daria la orden a la resistencia de calentar y el calentamiento se detendria al alcanzar la temperatura deseada. Como el aire es mal conductor, si bien la temperatura exterior era de $0^{\circ} \mathrm{C}$, la temperatura del fluldo no descendia de $30^{\circ} \mathrm{C}$. Para subsanar esta dificultad el tanque fue colocado en un criostato y a continuación el filtro. El equipo asi rediseñado fue probado con buenos resultados (Figura 1). 
3. DETBRHINACION CUANTITATIVA DE ACIDOS GRASOS POR CROMATOGRAFIA GASEOSA (GLC)

\subsection{Preparación de la nuestra}

Se colocaron 10 gotas de extracto graso con $40 \mathrm{ml}$ de una mezcla de metanol: benceno y $\mathrm{SO}_{4} \mathrm{~Hz}_{2}$ concentrado $3 \% \mathrm{P} / \mathrm{V}$ y se mantuvo a reflujo $30 \mathrm{min.} \mathrm{Seguidamente} \mathrm{se} \mathrm{dejó} \mathrm{enfriar} \mathrm{y} \mathrm{luego} \mathrm{se} \mathrm{extrajo}$ con $50 \mathrm{ml}$ de hexano, agitando 1 min. Se lavó hasta neutralidad con ClNa al $10 \%$ y naranja de metilo como indicador. Se secó con SOANaz anhidro. Se evaporó el hexano a baja presión, se hizo una dilución 1:10 en hexano y se inyectó $1 \mu l$ de la muestra en el cromatógrafo gaseoso.

\subsection{Equipo utilizado}

Las corridas cromatográficas se llevaron a cabo en un equipo Hewlett Packard modelo 5890 A. Las áreas correspondientes a cada pico se determinaron en forma relativa con un integrador Hewlett Packard 3392 .

\subsection{Condiciones de corrida}

Las condiciones de corrida en las que se encontró mejor resolución para una mezcla de cantidades equimoleculares de los éste- 
res metilicos de los ácidos linoleico, linolénico, oleico, palmitico y esteárico (patrones marca Sigma de 99,99 x de pureza) fueron un caudal de $\mathrm{N}_{2}$ de $20 \mathrm{ml} / \mathrm{min}$, una temperatura del inyector de $250^{\circ} \mathrm{C}$ y una temperatura de columna $188^{\circ} \mathrm{C}$. La columa usada fue 10\% SP 2330 en $100 / 120$ Chromosorb WAW de $1,70 \mathrm{~m}$ de largo y $0,3 \mathrm{~cm}$ de diámetro interno.

Los tiempos de retención de los componentes de la muestra original y de las fracciones sólidas y liquidas fueron coincidentes con los de los patrones utilizados en idénticas condiciones. Los cromatogramas se efectuaron por triplicado encontrando una alta reproducibilidad entre las repeticiones. El error en las áreas en todas las determinaciones resultó menor que $0,5 \%$.

Los ácidos grasos identificados fueron: 12:0 (laúrico), 14:0 (miristico), 16:0 (palmitico), 18:0 (esteárico), 18:1 (oleico) y $18: 2$ ( linoleico).

4. DETERMINACIOA CUANTITATIVA DE TRIGLICERIDOS POR CROHATOGRAFIA LIQUIDA DE ALTA PRESION (HPLC)

\subsection{Preparacion de la nuestra}

$40 \mu g$ de triglicéridos se disolvieron en $2 \mu 1$ de cloroformo. La disolución resultante se filtró con jeringa Luer Lock y filtros HF para solventes orgánicos. Luego se desgasificó en un sonicador durante 3 min. La muestra asi tratada estuvo en condiciones de ser inyectada. Los solventes constituyentes de la fase móvil recibieron el mismo tratamiento que las muestras, siendo filtrados a traves de un filtro Millipore adosado a una bomba de vacio. 


\subsection{Equipo utilizado}

Los experiencias fueron realizados con un cromatógrafo $11-$ guido Haters modelo 6000 A con sistema de mezclado de solventes; inyector universal de cromatografia liquida modelo U6R; detector UV-visible 450 Waters con longitud de onda variable. Los cromatogramas fueron impresos e integrados con un equipo Waters Data Module $M 730$.

\section{3 Condiciones de corrida}

La columna utilizada fue una microBondapak C18 Haters de $30 \mathrm{~cm}$ de longitud y $4 \mathrm{~mm}$ de diametro interno. La corrida se llevo a cabo a temperatura ambiente. El sistema de solventes utilizado consistió en una mezcla binaria de acetona, acetonitrilo ( $1: 2 \mathrm{~V} / \mathrm{V})$, caudal 1,1 ml/min. Todos los solventes eran de grado espectroscópico de la firma Carlo Erba.

El solvente usado para solubilizar triglicéridos determina la extensión con que las moleculas del soluto interaccionan con la fase móvil y ligandos hidrocarbonados (Singleton and Patteo, 1984). Las pruebas con los solventes habituales para lipidos tales como cloroformo, acetona, acetonitrilo-metanol (50,50), acetonitrilo-etanol $(80 / 20)$, hexano, etc, arrojaron como resultado que de todos estos solventes el que mantenia una relación lineal entre cantidad de muestra inyectada y respuesta (absorción de luz ultravioleta a $210 \mathrm{~nm}$ ) era el cloroformo.

La elección de la fase móvil se realizó corriendo en idén- 
ticas condiciones $2 \mu l$ do muestra en la concentractón ya especificada con los distintos sistemas de solventes empleados on la bibliografia para separar triglicéridos. Los resultados encontrados son coincidentes con los de Kimmey y Perkins (1884).

La detección fue realizada utilizando una longitud de onda de $210 \mathrm{~nm}$. Cada cromatograma fue repetido tres veces y las diferencius entre las áreas de los picos medidas por el integrador no fueron superiores al 1\%. Los valores que se informan son los promedios de estas medidas.

\subsection{Cuantificación de las áreas e identificación de conponentes}

Para cuantificar las áreas se conocen cuatro métodos: normalización de área (que supone que todos los componentes que eluyen en la muestra han sido detectados y con igual respuesta), standard interno, standard externo y una combinación de ambos llamado adición de standard (un constituyente de la muestra pero agregado en cantidades conocidas) (Johnson and Steverson, 1978). El integrador Haters Data Module M 730 utiliza el primer método.

Los tiempos de retención fueron calculados restándole al tiempo total que marcaba el integrador el tiempo que tarda en salir el volumen muerto (Done y col., 1874).

Para identificar los picos del cromatograma se usaron patrones de triglicéridos saturados marca Sigma y los métodos propuestos por Nawar (1985) y Takahashi y col. (1984,1985,1986). A cada pico constituyente de un cromatograma se le asigna un número equivalente de carbono ECN el que se calculó de la siguiente manera: 


$$
\mathrm{ECN}=\mathrm{Cn}-2 \mathrm{~N}
$$

donde $N$ es el numero de insaturaciones y $C n$ la longitud de cadena (Johnson y Stevenson, 1978).

Los acidos grasos insaturados (oleico y linoleico) constituyentes de los triglicéridos eluyen a tiempos menores que los que corresponden a la longitud de sus cadenas; ast el triglicérido tripalmitina tiene un tiempo de retención mayor en el mismo sistema que el correspondiente a trioleina.

En la práctica se ha visto que el ácido oleico se comporta como si su longitud de cadena fuera 15,5 (Frede y Thiele, 1988).

\section{CALORIHETRIA DIFERENCIAL DE BARRIDO (DSC)}

\section{1 Rquipo utilizado}

El equipo de análisis calorimétrico usado fue un calorimetro diferencial programado Du Pont 910 acoplado a un accesorio mecánico de enfriamiento y un analizador térmico modelo 99. Los diagramas calorimétricos fueron impresos en un registrador Hewlett Packard 7046 B.

5.2 Calibración del equipo

El equipo fue calibrado usando indio como patrón. 15 mg de 
indio se calentaron de $45^{\circ} \mathrm{C}$ a $170^{\circ} \mathrm{C}$ a la misma velocidad con que se realizaron las corridas calorimetricas de las fracciones obtenidas $\left(10^{\circ} \mathrm{C} / \mathrm{min}\right)$.

La celda del calorimetro se alineó electrónicamente mediante el coeficiente de calibración E. Este coeficiente es nominalmente $200 \mu W / m V$ (o adimensionalmente 1,0). El valor de E debe ser determinado para cada celda en particular. La fómula que permite calcularlo es la siguiente:

$\Delta \mathrm{H} \mathbf{m}$

$\mathrm{E}=$

60 A B $\Delta q a$

donde:

$\Delta H$ es el calor de fusión en joule/g (meal/mg)

m es la masa de muestra en mg

A el śrea del pico en $\mathrm{cm}^{2}$

B el tiempo base en $\mathrm{cm} / \mathrm{min}$

$\Delta q a$ es la sensibilidad on ordenadas on $\mathrm{mV} / \mathrm{cm}$

E es el coeficiente de calibración de la celda

El patrón de indio tiene un valor de $\Delta H=28,4 \mathrm{Joule} / \mathrm{g}$, una temperatura de fusión de $156,8^{\circ} \mathrm{C}$ y la masa posada fue $15,0 \mathrm{mg}$. Midiendo el área bajo la curva de fusión de indio $y$ con los valores de $B$ y $\Delta g_{a}$ se determinó antes de cada experiencia el valor de $E$. 


\subsection{Condiciones de corrida}

Muestras de 15-20 mg fueron colocadas en cápsulas de aluminio herméticamente selladas y fueron sometidas a un programa de temperatura que comprendia una isoterma a $-40^{\circ} \mathrm{C}$ de 5 min, un calentamiento de $-40^{\circ} \mathrm{C}$ a $80^{\circ} \mathrm{C}$ a una velocidad de $10^{\circ} \mathrm{C} / \mathrm{min} \mathrm{y}$ una isoterma final a $80^{\circ} \mathrm{C}$ de 2 min. En los diagramas se representa dQ/dt en ordenadas versus tiempo en minutos. La sensibilidad usada en ordenadas vario entre $0,414 \mathrm{mJoule} / \mathrm{seg}$ y $1,038 \mathrm{mJoule} / \mathrm{seg}$ sogún la cantidad y calidad de la muestra. Cada muestra se corrió por lo menos por triplicado.

\subsection{Interpretación de los termogramas}

La interpretación de los termogramas fue hecha con un modelo matemático desarrollado por Donovan (1975) para estudiar el comportamiento térmico de conalbumina. Segura y colaboradores (1990) en nuestro laboratorio lo aplicaron por primera vez a lipidos. El modelo supone que las mezclas complejas se pueden interpretar según sus componentes principales. Cada uno de estos componentes presentaria una distribución gaussiana. Todos los componentes sumados deben coincidir con la curva experimental. La expresión matemática propuesta que interpretó la curva experimental fue la siguiente:

$H=a * \exp \left(-(t-b)^{\wedge} 2 / \sigma\right)+c^{*} \exp \left(-(t-d)^{\wedge} 2 / \sigma\right)+\ldots$ 
la misma posee tantos términos como componentes se rebelen en el análisis ya se trate de un pico perfectamente individualizado o un hombro. H es la suma de las áreas de cada componente y coincide con la curva experimental. a, c, e, etc son las alturas de las curvas en la temperatura de pico; b, $d, f$, etc son las temperaturas de pico y o es el desvio standard.

Una vez obtenido el termograma se realizó la corrección por temperatura y se midieron las alturas de cada transición endotérmica cada $1^{\circ} \mathrm{C}$. Se construyb una tabla temperatura ( $\mathrm{T}$ ) altura (h). Como el número de parámetros a iterar en la función $H$ era muy grande ( $a, c, e$, etc $y b, d, f, e t c)$ se realizó primero un ajuste con un programa gráfico en Basic y luego a partir de esos parámetros la computadora encontró los valores que ajustaban mejor. El desvio standard en las temperaturas maximas resultó en todos los casos entre $0,1^{\circ} \mathrm{C}$ y $0,5^{\circ} \mathrm{C}$ mientras que on las alturas el valor era del orden del $2 \%$.

Cada término de la expresión era el área de un componente, entendiéndose por tal una solución sólida, eutéctico o pseudocompuesto. Con las temperaturas de pico (Tp) y las alturas calculadas, utilizando un programa estadistico (SYSTAT, módulo 3 ) se hallaron las áreas bajo las curvas de cada componente y el área total.

\subsection{Cálculo del calor de transición}

Los valores determinados de las áreas fueron empleados para obtener el $\Delta H$ de cada componente según la fórmula: 
$A\left(\mathrm{~cm}^{2}\right) E(\mathrm{~mW} / \mathrm{mV}) B(\mathrm{~min} / \mathrm{cm}) S y(\mathrm{mV} / \mathrm{cm}) 60(\mathrm{seg} / \mathrm{min})$

$\Delta H=$

\section{an (mg)}

donde Sy es la sensibilidad en $\mathrm{mV} / \mathrm{cm}$ en ordenadas, E es la constante de la celda de DSC que resultó ser igual a 0,20089 en la primer experiencia y 0,20800 on la segunda, B es la base tiempo (en absisas), $A$ es el área en $\mathrm{cm}^{2}$ y n la masa de la muestra en miligramos.

6. DIFRACTOMETRIA DE RAYOS $x$

\section{6 . 1 Equipo enpleado}

Para el estudio del polimorfismo de aceite de girasol hidrogenado y de las fracciones obtenidas por cristalización se utilizó un equipo Phillips 1730 adosado a un sistema de control do temperatura. Las muestras fueron colocadas en una cámara de refracción fabricada en nuestro laboratorio que permitia mantener constante la temperatura con una solución de etilenglicol 3:1 proveniente de un criostato Lauda UK 30 .

6.2 Condiciones de corrida

Se empleó radiación de cobre Kala2 con $40 \mathrm{Kv}, 20 \mathrm{~mA}$ y una 
velocidad de corrida de $1^{\circ} / \mathrm{m}$ in barriendo ángulos de $5^{\circ}$ a $25^{\circ}$.

Se realizaron diagramas de rayos $X$ de las muestras originales, de las fracciones ast como fueron obtenidas y de las fracciones fundidas y cristalizadas a diferentes velocidades. En todas las oportunidades el equipo fue calibrado con un patrón de silicio puro. Las muestras se corrieron por duplicado encontrandose repetitividad.

\subsection{Interpretación de los diagramas}

Los diagramas impresos en el registrador del equipo fueron interpretados mediante la ecuación de Bragg:

$$
\mathrm{n} \lambda=2 \mathrm{~d} \operatorname{sen} \theta
$$

donde $n \lambda$ es el camino que recorre el haz de luz incidente; $\lambda$ la longitud de onda; d la distancia interplanar y $\theta$ el ángulo de incidencia.

En los espectros se ha registrado intensidad en ordenadas $y$ ángulos de $2 \theta$ en abscisas. Para obtener el valor de $\theta$ se midieron los grados recorridos a partir de $5^{\circ}$ y se dividieron for dos. Para este sistema $n \lambda / 2$ resultó ser 1,54 A. A partir de este valor y conociendo el ángulo $\theta$ se calcularon las distancias d que definen las diferentes formas cristalinas. 


\section{ESPECTROSCOPIA INFRARROJA}

7.1 Preparación de la nuestra

Las muestras fueron preparadas en films puros entre dos ventanas de K Br.

7.2 Equipo utilizado

El espectrofotómetro empleado fue un Beckman IR 10 de doble haz.

7.3 Condiciones de corrida

Los espectros se obtuvieron barriendo longitudes de onda entre 600 y $4000 \mathrm{~cm}^{-1}$. Como patrón para la calibración del equipo fue empleada Trioleina de $99,95 \%$ de pureza (Sigma).

7.4 Interpretación de 108 diagranas

Lae áreas fueron calculadas en forma relativa tomando como total la suma de las áreas de los picos correspondientes a los dos isómeros. 
8. RESONANCIA haghetica nUClear (RMH)

8.1 Preparación de la nuestra

$20 \mathrm{mg}$ de cada muestra fueron disueltos en $\mathrm{Cl}_{4} \mathrm{C}$.

8.2 Equipo enpleado

El equipo utilizado fue un espectrómetro de resonancia magnética nuclear de $\mathrm{H}_{1}$ marca Varian EM-360 A.

8.3 Condiciones de corrida

Tetrametilsilano al $3 \%$ fue usado como referencia interna. Se emplé un filtro $0,05 \mathrm{seg}$; poder de radiofrecuencia 0,06 mGauss; tiempo de barrido 2 min; amplitud $100 * 6$; ancho de barrido $10 \mathrm{~Hz}$; final de barrido $\mathrm{O} \mathrm{Hz}$. 
Resultados

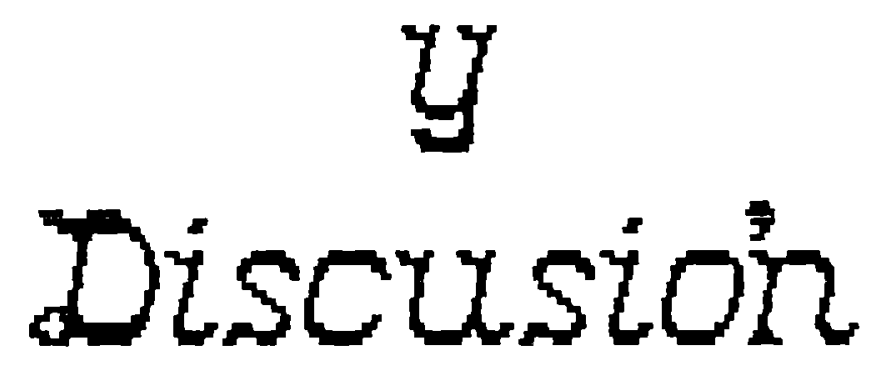




\section{BXPERIENCIAS PREVIAS AL FRACCIONAMIENTO}

A efectos de determinar las condiciones que se deberian emplear en el fraccionamiento de aceite de girasol hidrogenado se llevó a cabo una experiencia de cristalización estanca. Para ello se fundió fase grasa a $80^{\circ} \mathrm{C}$ en ocho recipientes pequeños. Los mismos se colocaron en un criostato regulado a $-30^{\circ} \mathrm{C}$. En todos los casos se registro la historia térmica de la muestra; al alcanzarse $20^{\circ} \mathrm{C}, \quad 15^{\circ} \mathrm{C}$ y $5^{\circ} \mathrm{C}$ se extrajeron dos recipientes por vez. Uno de ellos se tranafirió a un baño termostático cuya temperatura era próxima a la temperatura de extracción de la muestra $\left(22^{\circ} \mathrm{C}, 17^{\circ} \mathrm{C}\right.$ y $7^{\circ} \mathrm{C}$ ) y el otro se mantuvo a $5^{\circ} \mathrm{C}$ durante el término de 24 hs. En todos los casos las muestras se analizaron mediante calorimetria diferencial de barrido. Las condiciones empleadas fueron las siguientes:

Temperatura

Muestra

inicial $\left({ }^{\circ} \mathrm{C}\right)$

20

19

9

5 5
Temperatura

final

$\left({ }^{\circ} \mathrm{C}\right)$

80

80

80

80
Velocidad de calentamiento

$\left({ }^{\circ} \mathrm{C} / \mathrm{min}\right)$

1,2 y 3

\section{0}

10

10

10

10

Dado que las muestras fueron enfriadas en forma exponencial 

expresión:

$$
\begin{gathered}
\mathrm{T}_{A}-\mathrm{T}_{E} \\
\ln _{1}-\mathrm{T}_{E}
\end{gathered}
$$

donde:

TF es la temperatura del fluido refrigerante

TA y ta la temperatura y el tiempo en un punto cualguiera de la curva

Ti la temperatura inicial

Se escogió como temperatura inicial $80^{\circ} \mathrm{C}$, temperatura a la cual toda la muestra ha fundido. En todos los casos se alcanzó una velocidad de enfriamiento del orden de $10^{-2} \mathrm{seg}^{-1}$. Los resultados obtenidos en el primero y segundo dia se resumen en las Tablas 1 y 2. El análisis de las mismas señala que en todos los casos las muestras presentaron un pico y un hombro bien diferenciado. Las tempersturas de inicio del hombro disminuyeron al disminuir la temperatura a la que las muestras fueron retiradas del criostato. Considerando los valores absolutos de las áreas es notorio que al disminuir la temperatura de extracción el area del hombro aumenta asi como el área total, lo que indicaria una mayor cantidad de sólidos a menor temperatura. Durante el almacenamiento a $5^{\circ} \mathrm{C}$ cristalizaron triglicéridos con puntos de fusión comprendidos entre $20^{\circ} \mathrm{C}$ y $5^{\circ} \mathrm{C}$. Se observa además que los triglicéridos tienden a buscar las formas cristalinas más estables hecho que se verifica 
ron las modificaciones de las áreas relativas correspondientes a fico y hombro.

Tabla 1

Análisis calorimétrico realizado el priner dia

\begin{tabular}{|c|c|c|c|c|c|c|}
\hline \multirow{4}{*}{$\begin{array}{l}\text { Muestra } \\
\text { retirada } \\
a:\end{array}$} & \multirow{4}{*}{$\begin{array}{l}\text { Temperatura } \\
\text { inicial } \\
\text { Hombro } \\
\left({ }^{\circ} \mathrm{C}\right)\end{array}$} & \multirow{2}{*}{$\begin{array}{c}\text { Temperatura } \\
\text { Pico }\end{array}$} & \multicolumn{2}{|c|}{ Areas } & \multicolumn{2}{|c|}{ Areas } \\
\hline & & & Hombro & Pico & Hombro & Pico \\
\hline & & & & & & \\
\hline & & $\left({ }^{\circ} \mathrm{C}\right)$ & \multicolumn{2}{|c|}{$\left(m m^{2}\right)$} & \multicolumn{2}{|c|}{$(\%)$} \\
\hline $20^{\circ} \mathrm{C}$ & $20-22$ & 29 & 74,0 & 194,0 & 27,6 & 72,4 \\
\hline $15^{\circ} \mathrm{C}$ & $19-23$ & 31 & 73,2 & 178,9 & 29,0 & 71,0 \\
\hline $5^{\circ} \mathrm{C}$ & $8-23$ & 29 & 284,2 & 155,8 & 64,6 & 35,4 \\
\hline
\end{tabular}


Tabla 2

Análisis calorinétrico realizado el segundo dia

\begin{tabular}{|c|c|c|c|c|c|c|}
\hline \multirow{4}{*}{$\begin{array}{l}\text { Muestra } \\
\text { retirada } \\
\text { a: }\end{array}$} & Temperatura & Temperatura & \multicolumn{2}{|c|}{ Areas } & \multicolumn{2}{|c|}{ Areas } \\
\hline & inicial & Pico & Hombro & Pico & Hombro & Pico \\
\hline & Hombro & & & & & \\
\hline & $\left({ }^{\circ} \mathrm{C}\right)$ & $\left({ }^{\circ} \mathrm{C}\right)$ & \multicolumn{2}{|c|}{$\left(m m^{2}\right)$} & \multicolumn{2}{|c|}{$(\boldsymbol{z})$} \\
\hline $20^{\circ} \mathrm{C}$ & $5-13$ & 28 & 36,2 & 288,2 & 11,2 & 88,8 \\
\hline $15^{\circ} \mathrm{C}$ & $5-15$ & 26 & 55,3 & 301,2 & 15,5 & 84,5 \\
\hline $5^{\circ} \mathrm{C}$ & $5-15$ & 27 & 47,5 & 355,7 & 13,3 & 86,7 \\
\hline
\end{tabular}

Los resultados de esta experiencia sirvieron para diseñar la experiencia de fraccionamiento por temperatura. 


\section{FRACCIONAHIBNTO DB ACBITB DE GIRASOL HIDROGBHADO BN AUSBNCIA DE SOLVBHTES}

En la primer experiencia de fraccionamiento realizada se cristalizaron $50 \mathrm{Kg}$ de aceite hidrogenado de girasol (muestra 1) retirándose la totalidad del sólido acumulado en el filtro del equipo empleado a cada intervalo de temperaturas seleccionado (Materiales y Metodos punto 2.1). La experiencia se comenzb a $41^{\circ} \mathrm{C}$ y se finalizó a $19^{\circ} \mathrm{C}$ siguiendo escalones de $2^{\circ} \mathrm{C}$. A cada temperatura se extrajo además una porción del liquido presente en el tanque.

Una porción representativa del remanente del tanque se fraccionó en forma estática en un cristalizador sumergido en un baño a temperatura constante a $7^{\circ} \mathrm{C}$ y $3^{\circ} \mathrm{C}$.

La Figura 2 muestra el contenido de sólidos y liquidos para cada intervalo de temperaturas seleccionado. En todos los casos se consideraron los sólidos acumulados desde la temperatura de fusión hasta la temperatura de cristalización elegida. 


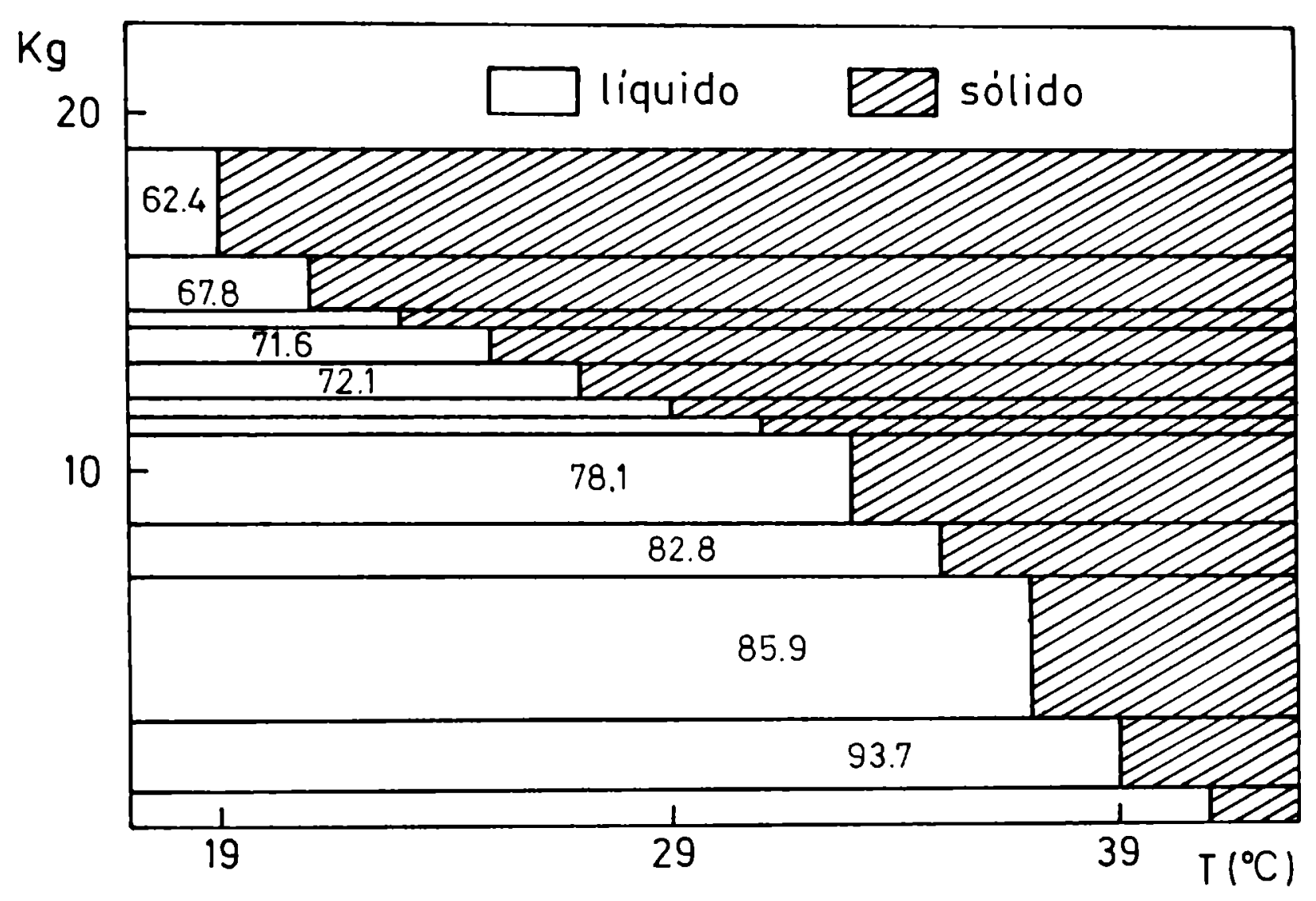

Figura 2. Sólidos y liquidos totales correspondientes a cada intervalo de temperaturas de cristalización seleccionado (Primera experiencia)

En la segunda experiencia de fraccionamiento se cristalizaron $27 \mathrm{Kg}$ de aceite hidrogenado de girasol (muestra 2) y se recogieron los sólidos cristalizados entre $38^{\circ} \mathrm{C}$ y $12^{\circ} \mathrm{C}$ a intervalos de $2^{\circ} \mathrm{C}$ y una porción representativa del liquido presente en el tanque a las mismas temperaturas. Los resultados obtenidos se presentan en la Figura 3. Al igual que en el caso anterior se consideraron los sólidos acumulados desde la temperatura de fusión hasta la temperatura de cristalización elegida. 


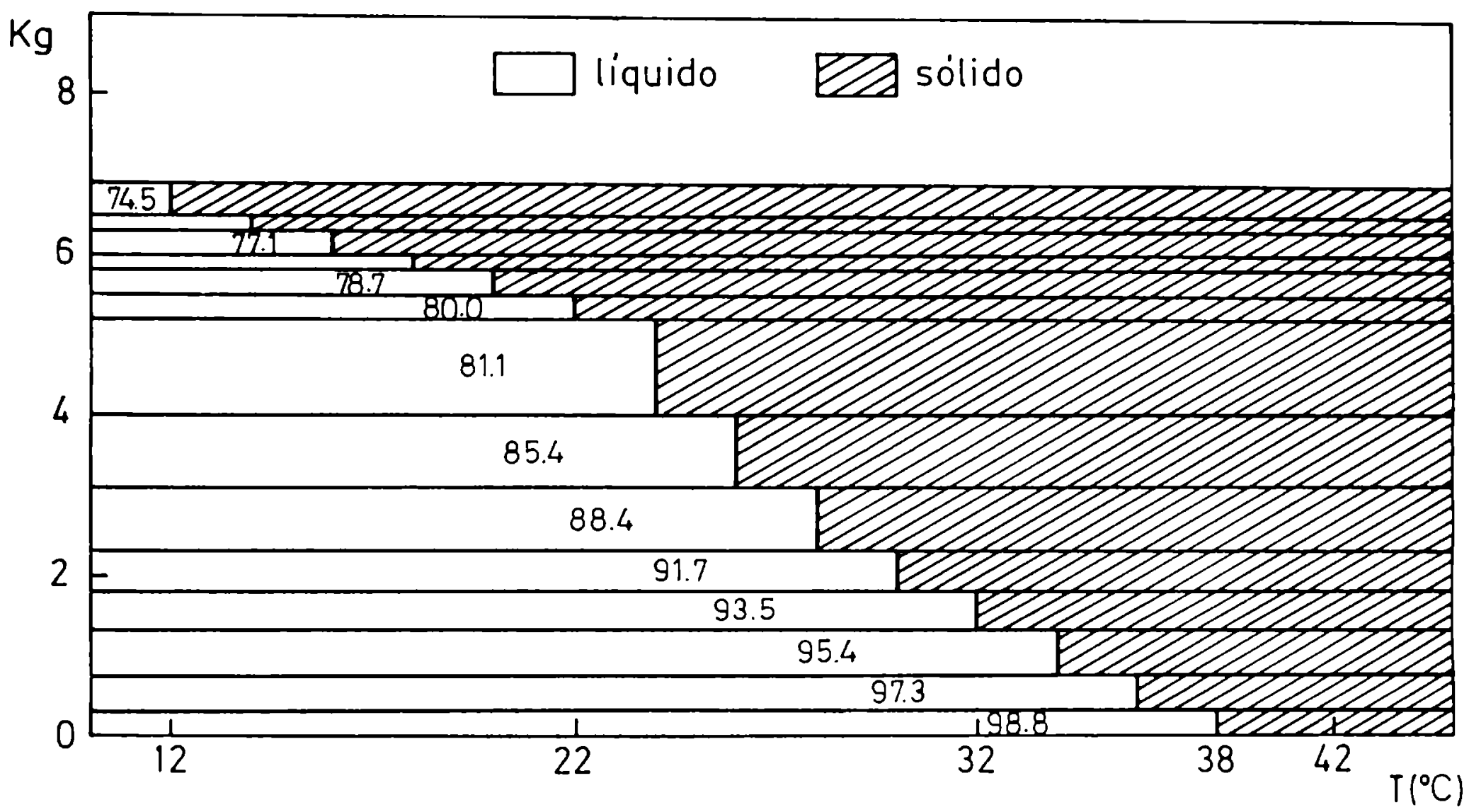

Figura 3. Sólidos y liquidos totales correspondientes a cada intervalo de temperaturas de cristalización seleccionado (Segunda experiencia)

El análisis de dichos resultados arroja como conclusión que el contenido de sólidos totales se incrementa al descender la temperatura de cristalización, hecho que ya habia sido observado en las experiencias preliminares. Por otra parte se encuentra que el contenido de sólidos totales es superior en la muestra 1 que en la muestra 2. Esto haria suponer que la hidrogenación procedió en mayor extensión en el primer caso.

Si analizamos la cantidad de sólidos que cristaliza en cada 
intervalo de temperatura empleado (Figura 4) observamos que en ambas experiencias se puede dividir el perfil de la figura en tres zonas.

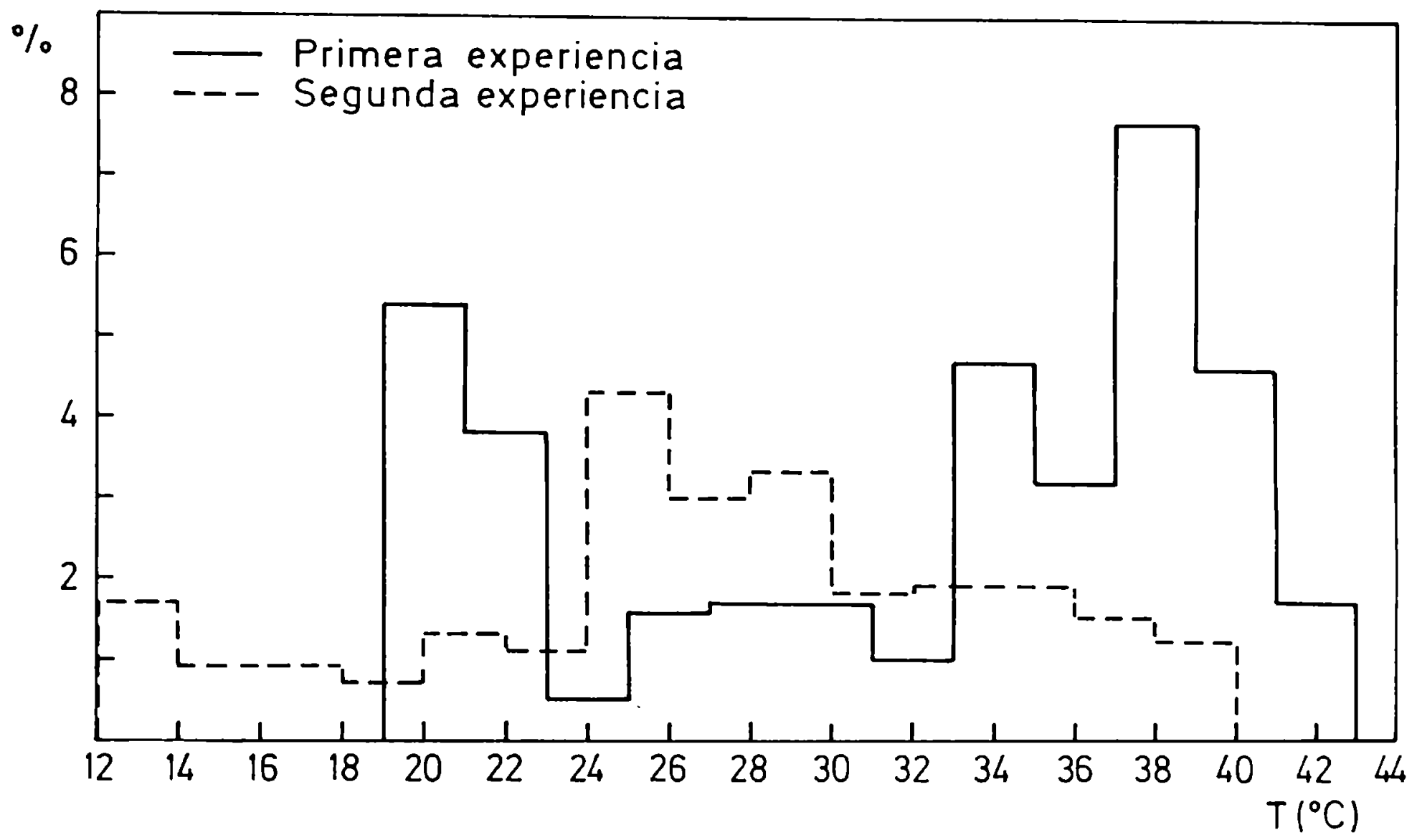

Pigura 4. Sólidos cristalizados en cada intervalo de temperatura

En la primera zona (por encima de $31^{\circ} \mathrm{C}$ ) en la muestra 1 se produce un aumento importante del contenido de sólidos de fracción en fracción mientras que en la muestra 2 este incremento es pequeno. La segunda zona (de $31^{\circ} \mathrm{C}$ a $23^{\circ} \mathrm{C}$ ) presenta un comportamiento inverso a la anterior. La muestra 2 aumenta en el contenido de sólidos en mucho mayor grado que la muestra 1 . La tercer zona comprende las temperaturas por debajo de $23^{\circ} \mathrm{C}$ y en ella se verifi- 
ca para ambas muestras el mismo comportamiento correspondiente a la primera zona. De acuerdo a estas figuras se puede esperar que la uuestra 1 contenga mayores porcentajes de triglicéridos saturados.

\subsection{Caracterización de las nuestras originales}

Para poder evaluar los cambios en la composición quimica y en el comportamiento térmico de las diferentes fracciones obtenidas en estas experiencias es necesario conocer las caracteristicas de las muestras originales. A continuación se resumen los resultados hallados al analizar los aceites hidrogenados de girasol que se emplearon.

2.1.1 Deterninación cuantitativa de ácidos grasos por cronatografia gascosa

El análisis de la muestra 1 arrojó la siguiente composición en ácidos grasos: palmitico $8,3 \%$, esteárico $11,5 \%$, oleico $76,4 \%$ y linoleico $3,7 \%$ y $0,1 \%$ de otros ácidos grasos tales como miristico y laúrico. Para la muestra 2 los resultados hallados fueron los siguientes: ácido palmitico $6,7 \%$, ácido esteárico $11,0 \%$ ácido oleico $68,8 \%$ y ácido linoleico $12,2 \%$ y un $0,2 \%$ de ácido miristico y laúrico. El aceite de girasol puro presentó un $6,7 \%$ de ácido palaitico, $3,8 \%$ de ácido estérico, $30,5 \%$ de ácido oleico, $57,8 \%$ de ácido linoleico, y $1,2 \%$ de otros ácidos grasos. La comparación de ambas muestras originales permite ver que en la muestra 1 el á- 
cido palmitico oe encuentra en mayor porcentaje que en la muestra 2 asi tambien como el ácido oleico, mientras que el ácido linoleico se halla en menor porcentaje. De acuerdo a estos resultados se esperaria que el punto de fusión de la muestra 1 fuera superior al de la muestra 2 lo que es concordante con el contenido de sólidos.

Ambos hidrogenados son más ricos en acidos estéarico y oleico gue el aceite sin hidrogenar y poseen mucho menor cantidad de ácido linoleico lo cual es esperable ya que en el proceso de hidrogenación el ácido linoleico reacciona con el hidrógeno para dar lugar a ácido oleico o esteárico. El contenido de ácido palmitico en cambio se mantuvo en el mismo orden.

2.1.2 Deterninación cuantitativa de trigliceridos por cronatografia liquida de alta presión

Uno de los objetivos planteados es la identificación tentativa de los trigliceridos presentes en cada fracción obtenida, para ello empleamos cromatografia liguida de alta presion. A efectos de poder asignar un determinado triglicérido o mezcla de triglicéridos a cada pico obtenido en un cromatograma ess necesario contar con patrones de triglicéridos que tengan probabilidad de hallarse presentes en la mezcla. Por esta razón realizamos corridas cromatográficas con patrones de triglicéridos saturados (SSS y PPP) y analizamos los resultados por el metodo matricial de Takahashi y colaboradores (1984, 1985, 1986). Ante la imposibilidad de contar con todos los patrones de triglicéridos necesarios se buscó una solución alternativa para ello. Analizamos aceites cuya 
composición en ácidos grasos y triglicéridos ya ha sido determinada y se compararon los resultados obtenidos con los existentes en bibliografia. De acuerdo a los resultados obtenidos por Dong y Di Cesare (1983), quienes emplearon una columna C18, fase móvil acetona: acetonitrilo $7: 3$ con un caudal de $2,0 \mathrm{~m} / / \mathrm{min}$, los aceites de algodón, girasol, matz y oliva poseen la composición en triglicéridos que figura a continuación:

Aceite

Tiempos de retención

$$
\text { (min) }
$$

$2,83,13,54,04,64,95,45,55,65,86,57,78,39,8$

oliva -- LLO LLP LOO PLO PPL OOO -- -- POO PPO SOO SOP SOS

maiz LLL LLO LLP LOO PLO _- -

palma $\ldots \ldots$ - $\ldots$ LOO PLO PPL OOO _.... POO PPO SOO SOP SOS

girasol LLL LLO LLP LOO -- - 000 -

Los resultados del análisis del aceite de oliva mencionados se obtuvieron mediante una corrida cromatográfica de alta resolución colocando tres columnas en serie; por esta razón se detectaron al final del cromatograna cuatro triglicéridos de tiempos de retención largos.

Abu-Hadeed y Kaotb (1988), Takano y Kondoh (1988), Takahashi y colaboradores (1884), Kimmey y Perkins (1983) entre otros han analizado diversos aceites vegetales con resultados coincidentes.

Estos aceites se analizaron en nuestro laboratorio encontrándose los resultados que se resumen en la Tabla 3. 
Tabla 3

Composición en triglicéridos de algunos aceites vegetales

Tiempo de retención

(min)

Aceite $\quad 2,8 \quad 3,6 \quad 4,6 \quad 5,9 \quad 6,3 \quad 6,8 \quad 7,4 \quad 7,7 \quad 8,1 \quad 8,9 \quad 10,9 \quad 11,7$

oliva -- LLO LLP LOO PLO PPL 000 -.

girasol LLL LLO LLP LOO - $\ldots$ - 000 - 00 -

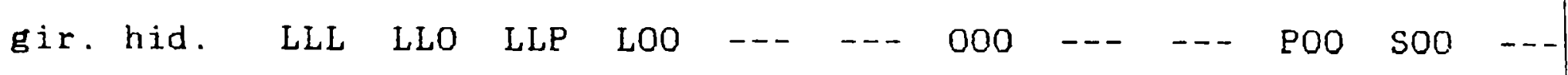

algodón LLL LLO LLP LOO - $\ldots 000$ - 00 POO

alg. hid. LLL LLO LLP LOO - $\ldots$ - 000 - $\ldots$ POO SOO maiz LLL LLO LLP LOO PLO -- -- SOL SPL

Los cromatogramas se realizaron con una sóla columna por esa razón no se detectaron los triglicéridos PPO y SOP que informaron Dong y Di Cesare en el aceite de oliva. Las diferencias en los tiempos de retención que se observan entre los valores que se presentan en la Tabla 3 y los hallados en bibliografa se deben a diferencias en el flujo de los solventes empleados. El flujo utilizado en las corridas cromatográficas efectuadas en nuestro laboratorio fue $1,3 \mathrm{ml} / \mathrm{min}$. Un flujo de $2,0 \mathrm{ml} / \mathrm{min}$ como el empleado por Dong y Di Cesare elevaba la presión en la columa a un valor cercano a 3000 Psi considerado como el limite de seguridad. Las correcciones por diferencjas de flujo de los tiempos de retención arrojaron los resultados hallados por los mencionados autores. La 
concordancia entre los cromatogramas presentados por ellos y los realizados en nuestro laboratorio nos permitio asignar a cada pico un triglicerido mayoritario.

Si se modifica la relación de solventes en la fase móvil se modifican los tiempos de retención. Cuando estos aceites se analizaron empleando como fase móvil acetona:acetonitrilo $1: 2$ (sistema de solventes empleado para analizar todas las muestras recogidas en la experiencia de fraccionamiento dada la mejor resolución hallada) los tiempos de retención encontrados fueron mayores: 5, 1; 6,$5 ; 8,8 ; 12,5 ; 16,4 ; 21,5 ; 29,1$ y 39,8 para LLL, Llo, LlP, L0O, 000, $\mathrm{POO}$, SOO y SSO respectivamente. Si bien los tiempos de retención son muy diferentes a los encontrados con una mezcla de elución $7: 3$ los perfiles presentados por 18 muestra no se modifican; es por esta razón que podemos mantener las asignaciones hechas proviamente.

Otra forma de verificar si se ha asignado correctamente un triglicérido a un pico determinado es calcular la composición aproximada de los aceites analizados. De acuerdo con Nawar (1985) este cálculo se efectúa multiplicando los porcentajes en que se hallan presentes los ácidos grasos en el aceite. Sin embargo se ha abservado en la naturaleza que es rara la ocurrencia de un triglicérido formado por ácidos grasos idénticos si el porcentaje de ese ácido graso en la muestra es inferior al 65\%. A continuación se resumen las composiciones en acidos grasos que figuran en bibliografia de los aceites mencionados anteriormente. 


$\begin{array}{lrrrrrrr}\text { Algodón } & 24,5 & 1,3 & 1,8 & 16,3 & 55,4 & \ldots & 0,7 \\ \text { Girasol } & 6,7 & --- & 3,8 & 30,5 & 57,8 & \ldots & 1,2 \\ \text { maiz } & 13,6 & 0,4 & 1,7 & 37,3 & 46,0 & 0,6 & 0,4 \\ \text { oliva } & 10,0 & 1,5 & 1,7 & 66,5 & 20,3 & \ldots\end{array}$

Según estos contenidos en ácidos grasos y siguiendo el procedimiento de Nawar los triglicéridos más probables para el aceite de oliva serán L0O, 000, y POO los cuales eluyen en ese orden cuando son analizados por Cromatografia Liguida. En el cromatograma que se obtuvo en nuestro laboratorio estos tres triglicéridos mencionados correspondieron a los picos mayores. Graciani Constante ( $198^{\prime} 7$ ) estudió extensamente la separación de triglicéridos en aceite de oliva encontrando resultados ooncordantes con los nuestros. En sus cromatogramas sólo los mencionados triglicéridos presentaron picos importantes. Los restantes presentaron picos menores.

En orden de probabilidad decreciente según datos bibliográficos los triglicéridos mayoritarios presentes en el aceite de maiz deben ser LOO, PLO, LLO, SOL, LLP, SPL y un pequeño pico de LLL. En la Figura 5 se presenta el cromatograma obtenido en nuestro laboratorio. La figura es concordante con la composición calculada. 


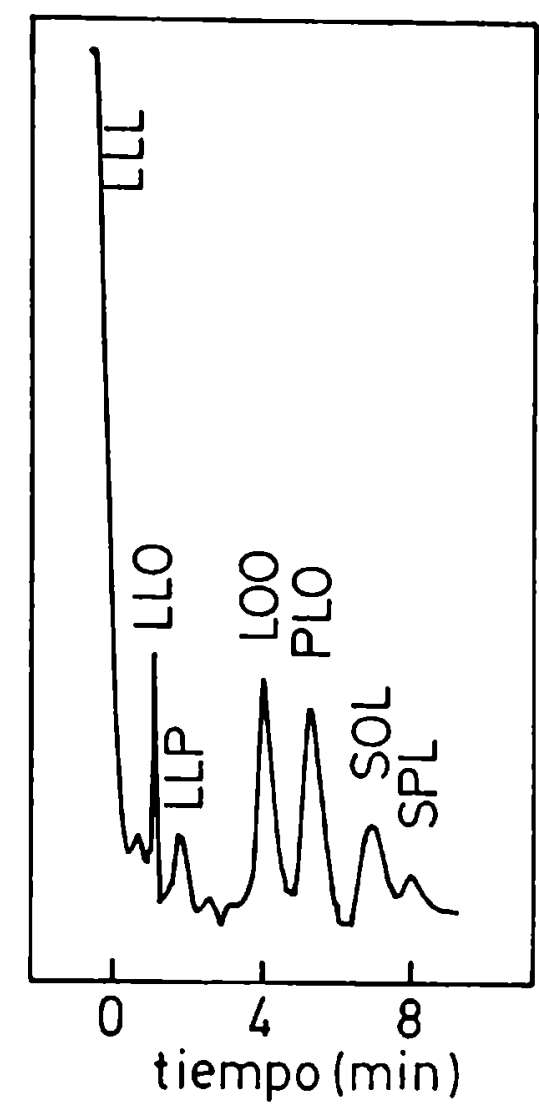

Figura 5. Cronatograna de aceite de naiz. Fase nóvil acetona: acetonitrilo $7: 3$; flujo $1,3 \mathrm{nl} / \mathrm{nin}$

Las Figuras 6 a y b corresponden a dos cromatogramas de aceite de algodón, sin hidrogenar e hidrogenado. En el aceite sin hidrogenar, según la composición informada, los triglicéridos con mayor probabilidad de formación son LLP, LLO, LOO y en menor proporción deberian encontrarse 000 , P0O y S00. En el aceite hidrogenado se produce una notable disminución en los picos correspondientes a LLO, LLP y LOO y un aumento en la probabilidad de los picos 000 , P00 y s00. Es claro en las figuras el cambio on la composición química que se produce durante la hidrogenación. 


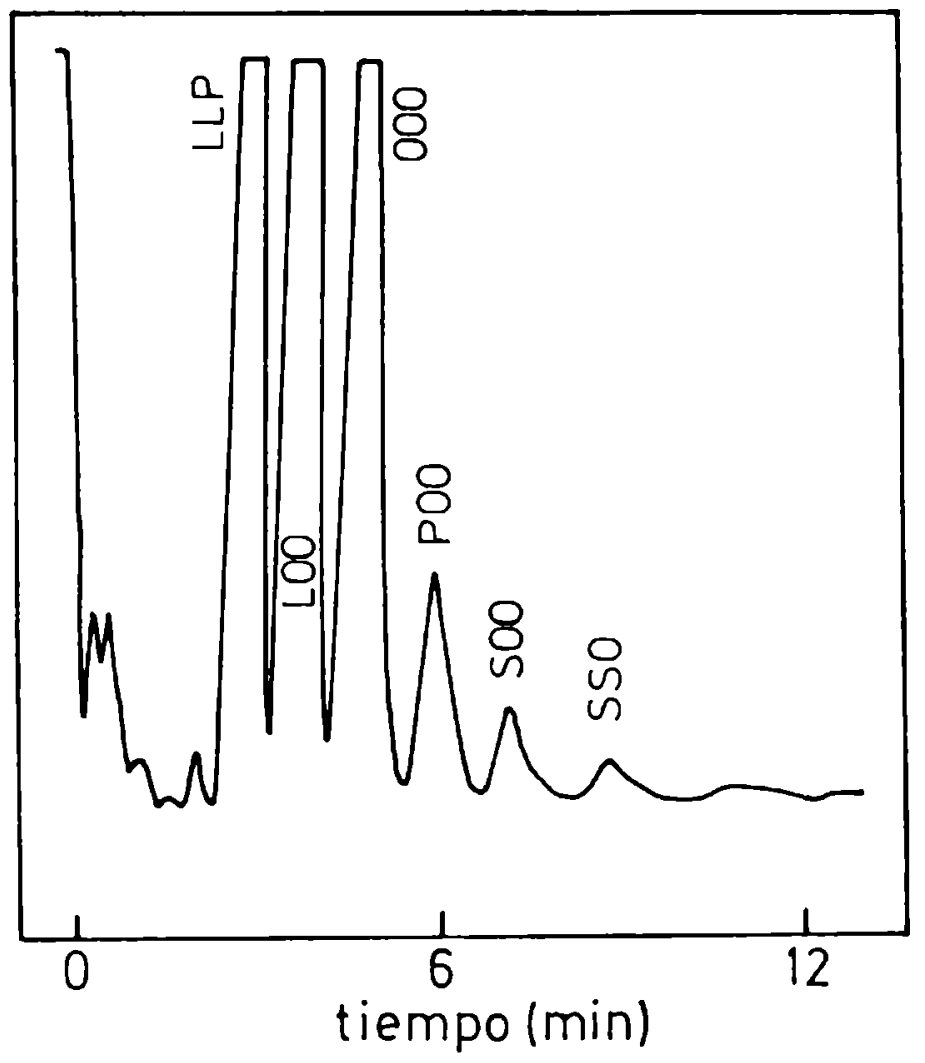

(a)

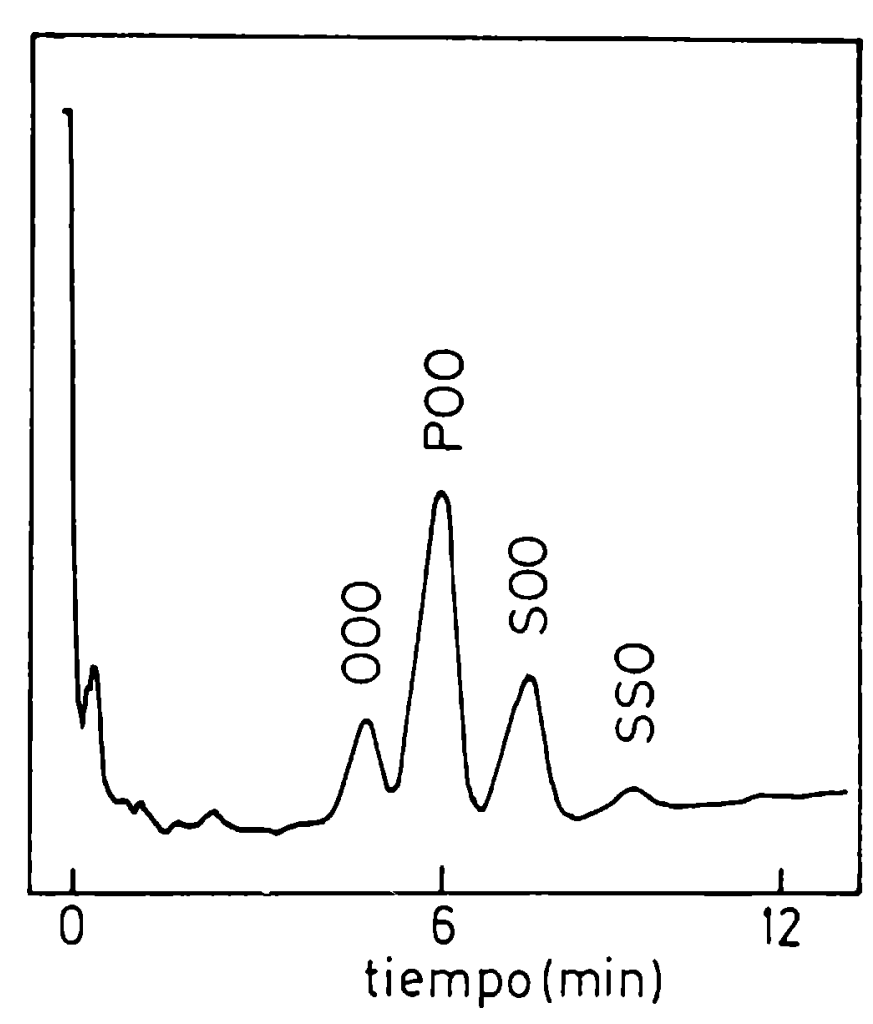

(b)

Figura 6. Crongtograna de aceite de algodón (a) y aceite de algodón hidrogenado (b). Se emplearon iguales condiciones que en la figura 5

Cromatogramas de aceite de girasol fueron analizados en forma semicuantitativa (Materiales y Métodos sección 4.4). El aceite de girasol hidrogenado arrojó la siguiente composición en triglicéridos: $3,7 \%, 21,7 \% ; 50,5 \%, 20,6 \%$, y $2,1 \%$ para los picos de tiempos de retención 5,$9 ; 7,4 ; 8,9 ; 10,9$ y 11,7 min correspondientes a 
L00, 000, POO, SOO y SSO respectivamente (Figura 7). El cromatograma de aceite de girasol normal presentó cuatro picos principales de tiempos de retención 3,$6 ; 4,6 ; 5,9$ y 7,4 min los gue representaron un $37,2 \%, 39,4 \%, 16,6 \%$ y $1,2 \%$ respectivamente (Figura 8 ). La comparación de ambos cromatogramas destaca el hecho que en el aceite hidrogenado el pico de 8,9 min es el mayor mientras que en el aceite sin hidrogenar es muy pequeño y que los picos de 3,6; 4,6 y 5,8 min disminuyen en forma notable aumentando los picos do 7,$4 ; 10,9$ y 8,9 min este ultimo anteriormente mencionado los cuales eran sumamente pequeños en el aceite sin hidrogenar.

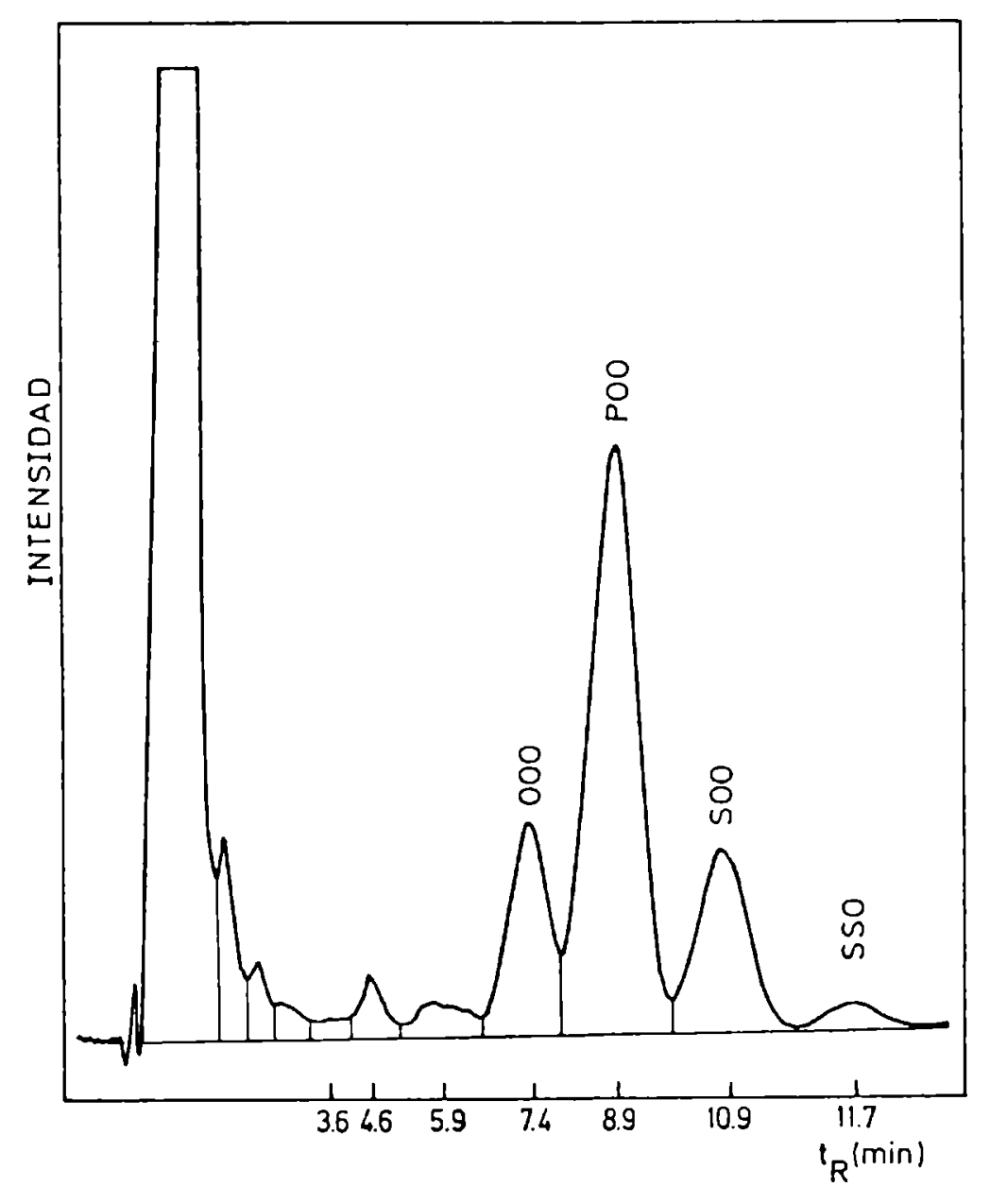

Figura 7. Cronatograna de aceite de girasol hidrogenado. Se enplearon iguales condiciones que en la figura 5 


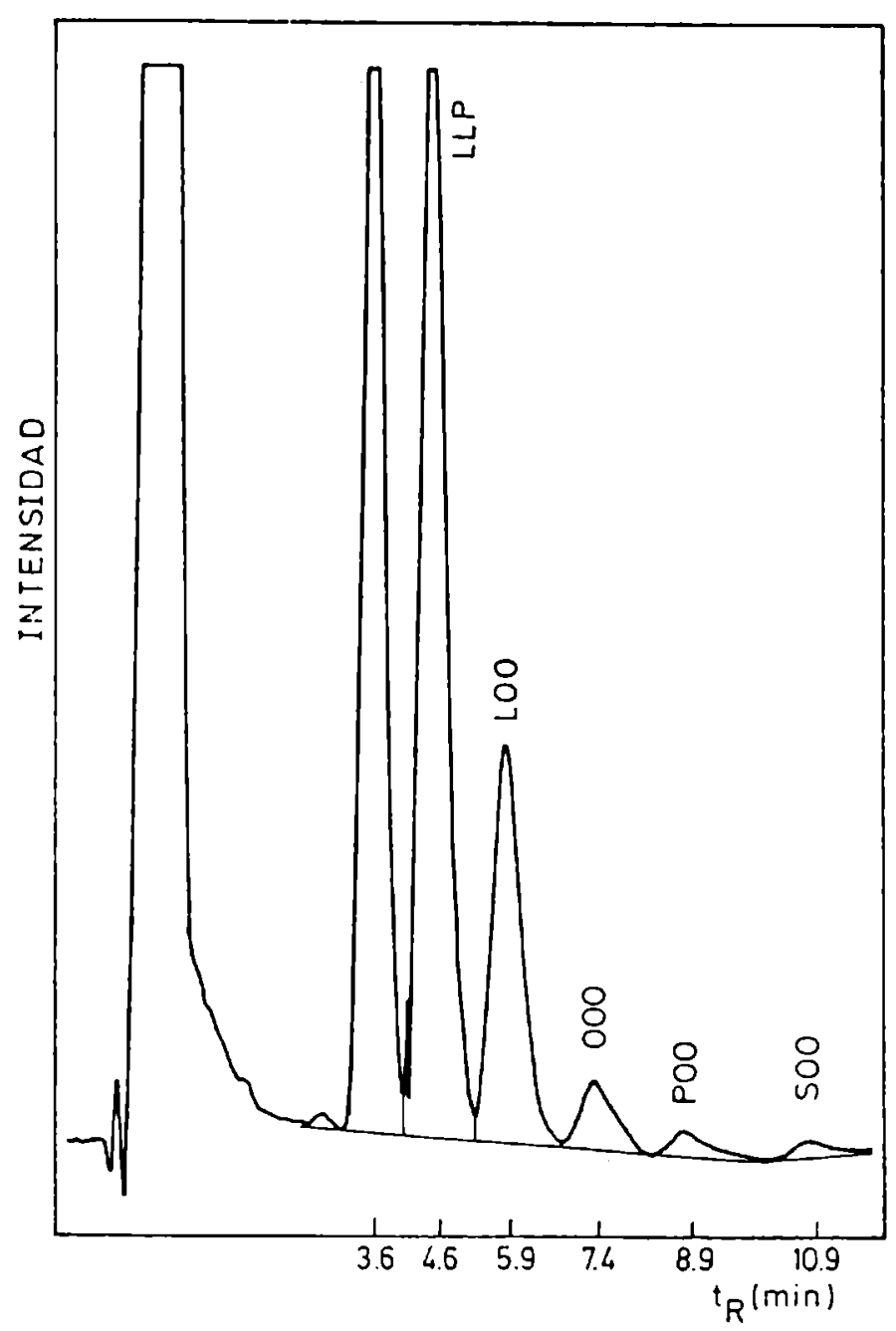

Figura 8. Cronatograma de aceite de girasol normal. Se enplearon iguales condiciones que en la figura 5

Lo discutido anteriormente nos permite estar seguros de nuestro sistema de análisis y asignación de picos. Por lo tanto procedamos a analizar las muestras empleadas en las experiencias de fraccionamiento (muestra 1 y 2 ). Los resultados obtenidos se presentan en la Tabla 4. 
Tabla 4

Conposición en triglicéridos de las nuestras originales

\begin{tabular}{|c|c|c|c|c|c|c|c|}
\hline & LLO & LLP & LOO & 000 & POO & s00 & SSO \\
\hline \multirow[t]{2}{*}{ Muestra } & \multicolumn{7}{|c|}{ Tiempos de rete } \\
\hline & 6,5 & 8,8 & 12,5 & 16,4 & 21,5 & 29,1 & 39,8 \\
\hline 1 & 0,2 & 2,0 & 4,2 & 7,3 & 25,5 & 48,0 & 12,0 \\
\hline 2 & 0,3 & 2,8 & 18,1 & 39,5 & 29,4 & 7,0 & 0,8 \\
\hline
\end{tabular}

La comparación entre ambos aceites hidrogenados permite ver que la muestra 2 contiene una mayor proporción de los triglicéridos LOO, 000 y POO y un menor porcentaje de SSO y SOO. De acuerdo a esto podria esperarse un mayor punto de fusión en la muestra 1. Estos resultados son coincidentes con la composición de ácidos grasos previamente determinada asi como con el contenido de sólidos.

\subsubsection{Calorinetria diferencial de barrido}

Para estudiar el comportamiento térmico de las muestras originales: diferentes puntos de fusión de los constituyentes presentes, posible polimorfismo $\theta$ interacciones entre los diferentes 
triglicéridos (formación de soluciones sólidas, pseudocompuestos o eutécticos) se realizaron estudios de calorimetria diferencial de barrido. En la Figura 9 se presentan los diagramas obtenidos al interpretar matematicamente los termogramas correspondientes a las muestras originales con el fin de compararlos.

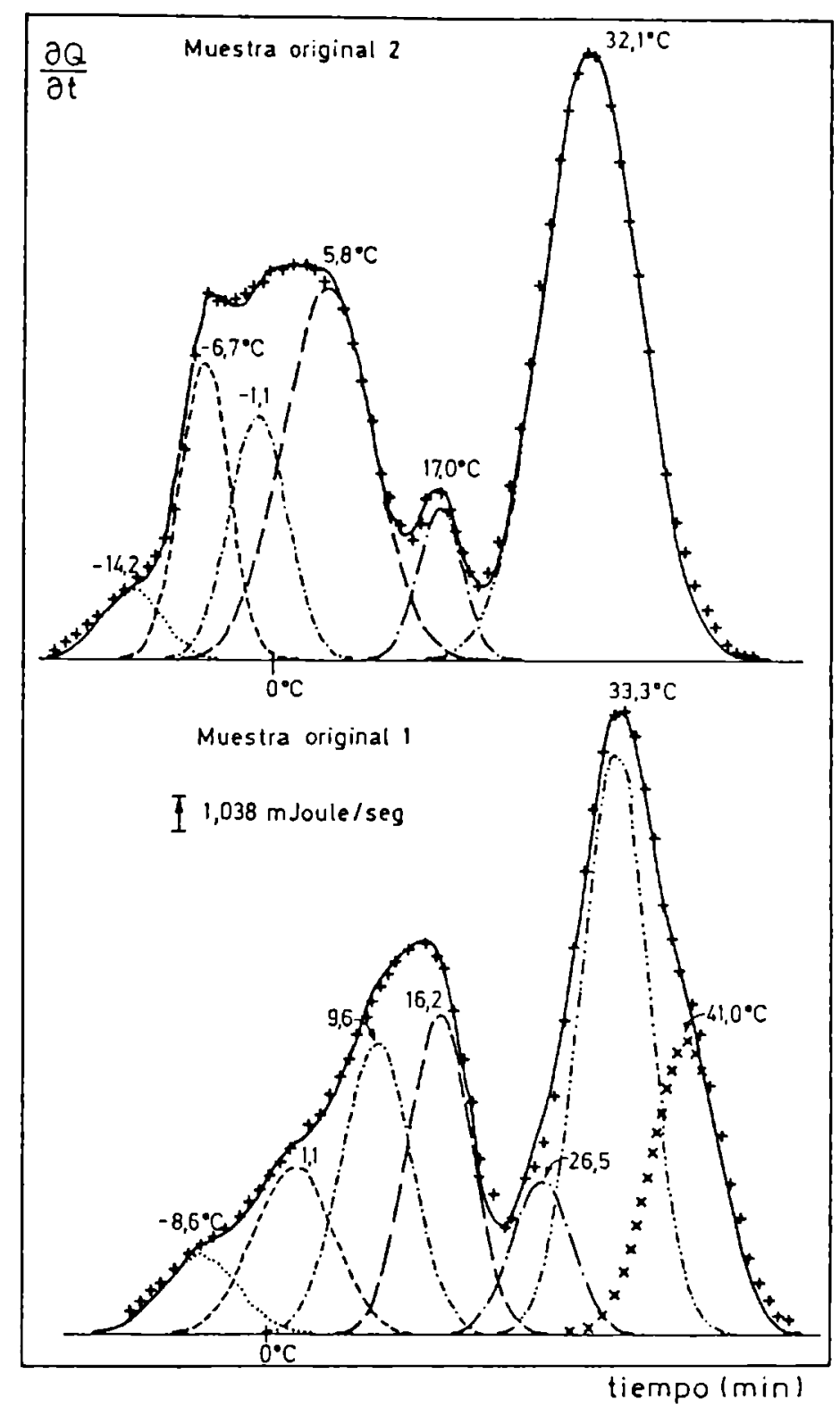

Figura 9. Ternogranas interpretados natenaticamente correspondientes a las muestras originales 
Los termogramas que se presentan en la Figura 9 son directamente comparables ya que las curvas representan el área por gramo de muestra empleada. Resulta evidente que los perfiles de ambas muestras son diferentes entre si. En la muestra 1 se separaron sólo dos endotermas mientras que en la muestra 2 se detectaron tres. Asi mismo la forma que presenta la primer endoterma en ambos termogramas es diferente; la endoterma de mayor temperatura en cambio es más similar. Por otra parte en la muestra original 1 las temperaturas de pico de los distintos constituyentes resultaron superiores a las correspondientes de la muestra 2 . En la muestra 1 el pico de mayor temperatura representa un porcentaje mayor respecto del área total que en la muestra 2 (53\% y $45 \%$ respectivamente).

Otras diferencias detectadas en las muestras surgen de la aplicación del método matemático descripto en Materiales y Métodos (sección 5.4). Los resultados obtenidos se resumen en la Tabla 5. 
Tabla 5

Calorinetria diferencial de las nuestras originales

\begin{tabular}{|c|c|c|c|c|c|c|c|c|}
\hline (1) & \multicolumn{5}{|c|}{ Primer pico } & \multicolumn{3}{|c|}{ Segundo Pico } \\
\hline Constituyente & 1 & 2 & & 3 & 4 & 5 & 6 & 7 \\
\hline Temperatura & $-8,8$ & 1, & & 8,6 & 16,2 & 26,5 & 33,3 & 41,0 \\
\hline Area $\left(\mathrm{mm}^{2}\right)$ & 67,9 & 155, & & 228,0 & 206,2 & 95,1 & 428,7 & 216,6 \\
\hline Area $(\%)$ & 4,9 & 11 , & & 16,3 & 14,7 & 6,8 & 30,7 & 15,5 \\
\hline$\Delta H($ Joule/g) & 4,2 & 9, & & 14,2 & 13,0 & 5,8 & 26,8 & 13,4 \\
\hline$\Delta H$ Pico & 41,0 & & & & & 46,0 & & \\
\hline$\Delta H$ total & 87,1 & & & & & & & \\
\hline (2) & & imer $p$ & ico & & Segundo & pico & Tercer & pico \\
\hline T'emperatura & $-14,2$ & $-6,7$ & $-1,1$ & 5,8 & 17 . & & & 2,1 \\
\hline $\operatorname{Area}\left(m m^{2}\right)$ & 54,3 & 159,8 & 154,0 & 341,9 & 67 , & & 636 & 6,3 \\
\hline Area $(\%)$ & 3,8 & 11,3 & 10,8 & 7 24,2 & 4 , & & & 5,0 \\
\hline$\Delta H($ Joule $/ g)$ & 3,3 & 10,0 & 9,6 & 21,3 & 4, & & & 9,8 \\
\hline$\Delta H \quad P i=0$ & 44,4 & & & & 4, & & & 9,8 \\
\hline$\Delta H$ total & 88,3 & & & & & & & \\
\hline
\end{tabular}

Del análisis de la mencionada Tabla surge que los constituyentes de cada endoterma en ambas muestras resultan diferentes 
entro si en puntos de fusión y áreas relativas; asi mismo su numero es superior en la muestra 1. La primer endoterma de la muestra 1 es asimétrica y pudo ser ajustada mediante la contribución de cuatro constituyentes de diferente peso. Si bien los mismos no se separan claramente en la endoterma original es posible visualizar en la misma la existencia de hombros. El ajuste de la curva mediante tres constituyentes aumenta el error en tanto que un numero mayor de los mismos no permite obtener una mejor aproximación. Al segundo pico contribuirian tres constituyentes indicados por diferencias en la pendiente de la endoterma original. El primer pico de la muestra 2 presenta un perfil ajustable con cuatro componentes en tanto que el pico de temperatura máxima $32,1^{\circ} \mathrm{C}$ pudo ser interpretado como conformado por un único constituyente. El hecho que los termogramas se ajusten a la sumatoria de un número de componentes superior a los visibles en forma directa, indicaria la presencia de fracciones de triglicéridos con puntos de fusión cercanos no diferenciables en las condiciones empleadas.

Según estos diagramas calorimétricos y considerando que solidifica todo lo que deberla solidificar y que además cada endoterma representa la fusión de un componente distinto el contenido de sólidos de la muestra 1 a $19^{\circ} \mathrm{C}$ es del $57,7 \%$ y el de la muestra 2 a $12^{\circ} \mathrm{C}$ de $49,7 \%$. Experimentalmente obtuvimos en el fraccionamiento de estas muestras a las temperaturas mencionadas valores de $37,6 \%$ y $25,5 \%$ respectivamente. Las diferencias encontradas al analizar las muestras originales por los métodos ya discutidos podrian deberse a las siguientes causas:

- los resultados encontrados por calorimetria diferencial de barri- 
do y por fraccionamiento no son comparables dado que las historias térmicas de las muestras fueron diferentes. Al retirar los solidos en la experiencia de fraccionamiento, la composición inicial de la muestra fue cambiando de fracción en fracción.

- la comparación entre los resultados obtenidos al analizar las muestras originales por cromatografia liguida de alta presión y calorimetria diferencial de barrido pone de manifiesto que solo cinco triglicéridos se encuentran en una proporción mayor al $3 \%$ (Tabla 4), mientras que en los diagramas interpretados matemáticamente se presentan un número de componentes mayor ( $T a b l a ~ 5$ ). Dada las distintas solubilidades de los triglicéridos ontre si, manifiesta en los diagramas de fase presentados por Gibon (1986) y otros autores, es posible suponer que cada pico correspondiente a la interpretación matemática de los diagramas obtenidos por calorimetria diferencial de barrido puede estar formado por más de un triglicérido. Pueden ocurrir interacciones entre algunos pares de triglicéridos presentes en el aceite de girasol hidrogenado que fraccionamos que conduzcan a este resultado, por ejemplo la formación de eutécticos entre EEE/SOS y SEE/SOS. Si estos pares se encuentran en la composicón eutéctica fundirán como un compuesto puro, asi también lo harán aquellos pares que formen soluciones sólidas de rango extendido de temperatura o que se encuentren en proporción adecuada para formar pseudocompuestos.

-por otra parte, la técnica de cromatografia liquida empleada para analizar la composición quimica de las muestras no permite separar isómeros geométricos. Teniendo en cuenta la historia previa de las 
muestras es lógico suponer que durante la hidrogenación se produzca isomerización. Los isómeros naturales cis y los trans producidos durante el procesamiento del aceite tienen puntos de fusion muy diferentes pero igual tiempo de retención, es decir eluyen en el cromatograma como un único pico pero aparecen como dos picos diferentes al fundir.

-además los calores de fusión de los triglicéridos puros son diferentes según de cual de ellos se trate y en que forma cristalicen; asi, SSS en su forma $\beta$ tiene un calor del orden de dos veces el de 000 cristalizada en la misma forma. Por esta razón un pico presente en pequeña cantidad en el cromatograma puede contribuir con un área importante al termograma. Asi mismo algún pico podria corresponder a una transformación polimórfica aunque esto no es muy probable.

Todos estos factores hacen que el comportamiento de fusión de estas mezclas resulte muy complicado.

\subsubsection{Difractonetria de rayos $X$}

Con el objeto de identificar las formas polimórficas presentes en las muestras originales se realizo una difractometria de rayos $X$. 


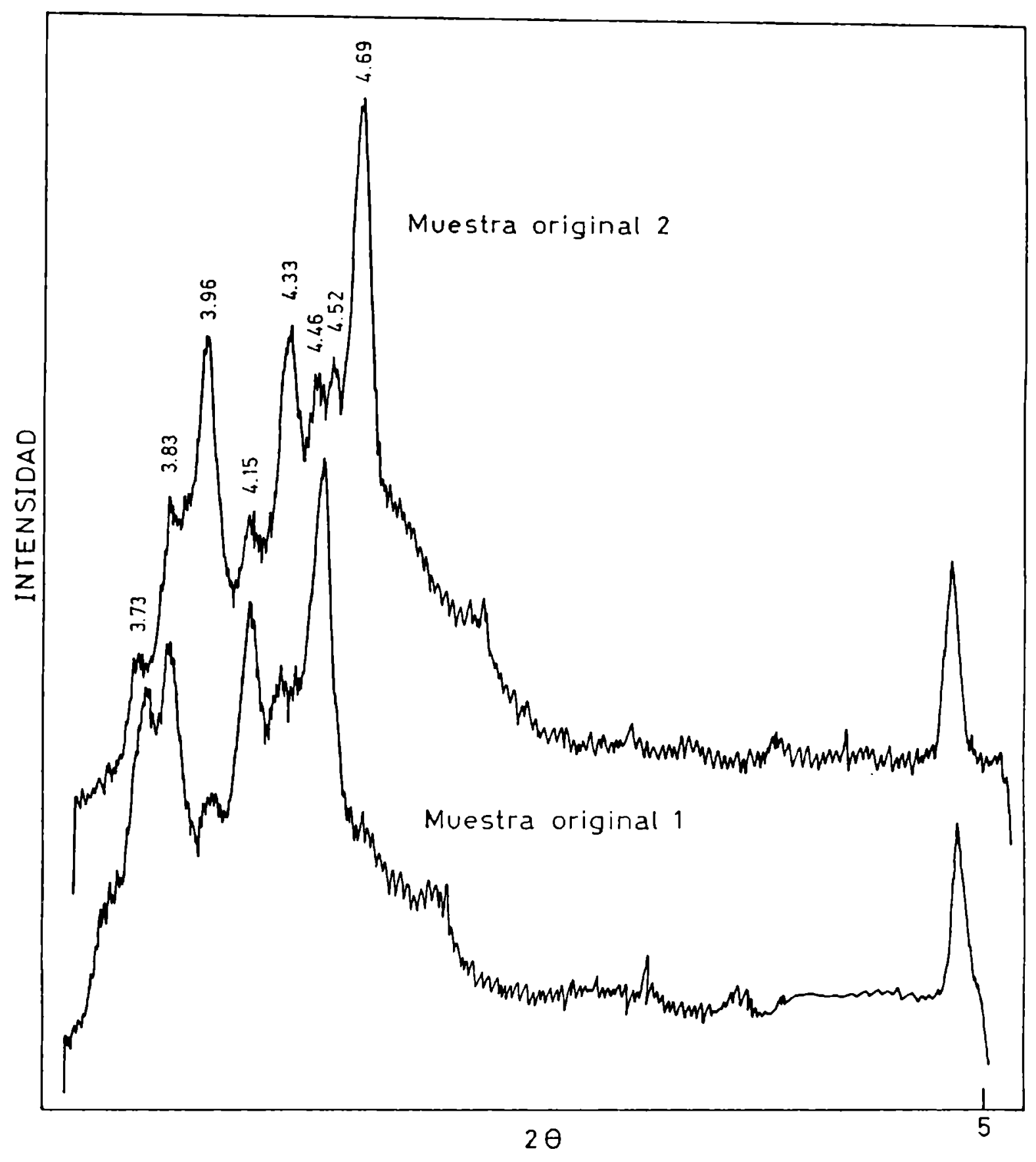

Figura 10. Diagranas de rayos $X$ de las muestras originales

El análisis de las mismas arrojó los siguientes resultados (Figura 10): en la muestra 1 se encontraron cuatro líneas principales a $3,7 \mathrm{~A} ; 3,8 \mathrm{~A} ; 4,1 \mathrm{~A}$ y $4,5 \mathrm{~A}$ mientras que en la muestra 2 
se observaron tres a 3,9 A; 4,3 A y 4,7 A y cinco ineas menores a $3,7 \mathrm{~A} ; 3,8 \mathrm{~A} ; 4,1 \mathrm{~A} ; 4,4 \mathrm{~A}$ y 4,5 A. Como hemos explicado previamente según Lutton (1955) las formas $\beta^{\circ}$ presentan dos a tres 1 ineas fuertes a 4,2 A y 3,5 A mientras que las formas $\beta$ presentan una linea a 4,6 A, la que usualmente es la más fuerte. Las pequeńas diferencias observadas en las muestras 1 y 2 en las lineas 3,7 A y 3,8 A respecto de 3,5 A podria deberse a la presencia de distintas formas $\beta^{\circ}$. La muestra 2 presenta además una línea a 4,7 A lo que indicaria la presencia de cristales $\beta$ o según la clasificación de Deroanne (1976) corresponderia a una forma intermediaria entre ambas. Ninguno de los dos diagramas de rayos $\mathrm{X}$ corresponde a los obtenidos con triglicéridos puros en las formas $\beta^{\circ} \circ \beta^{\circ}$ Esto apoya la suposición de que existe una mezcla de diferentes formas polimórficas.

\subsubsection{Espectroscopia Infrarroja}

Con el objeto de cuantificar el contenido de isómeros trans de las muestras originales se realizaron estudios de espectroscopia infrarroja. El aceite de girasol normal presenta un pico a 720 $\mathrm{cm}^{-1}$ correspondiente al isómero C 18:1 cis. Si este aceite es hidrogenado este pico se reduce apareciendo el correspondiente al isómero C 18:1 trans a $980 \mathrm{~cm}^{-1}$ (Eigura 11). Las muestras 1 y 2 presentaron un contenido de isomeros trans de $43,4 \%$ y $44,2 \%$ respectivamente. 


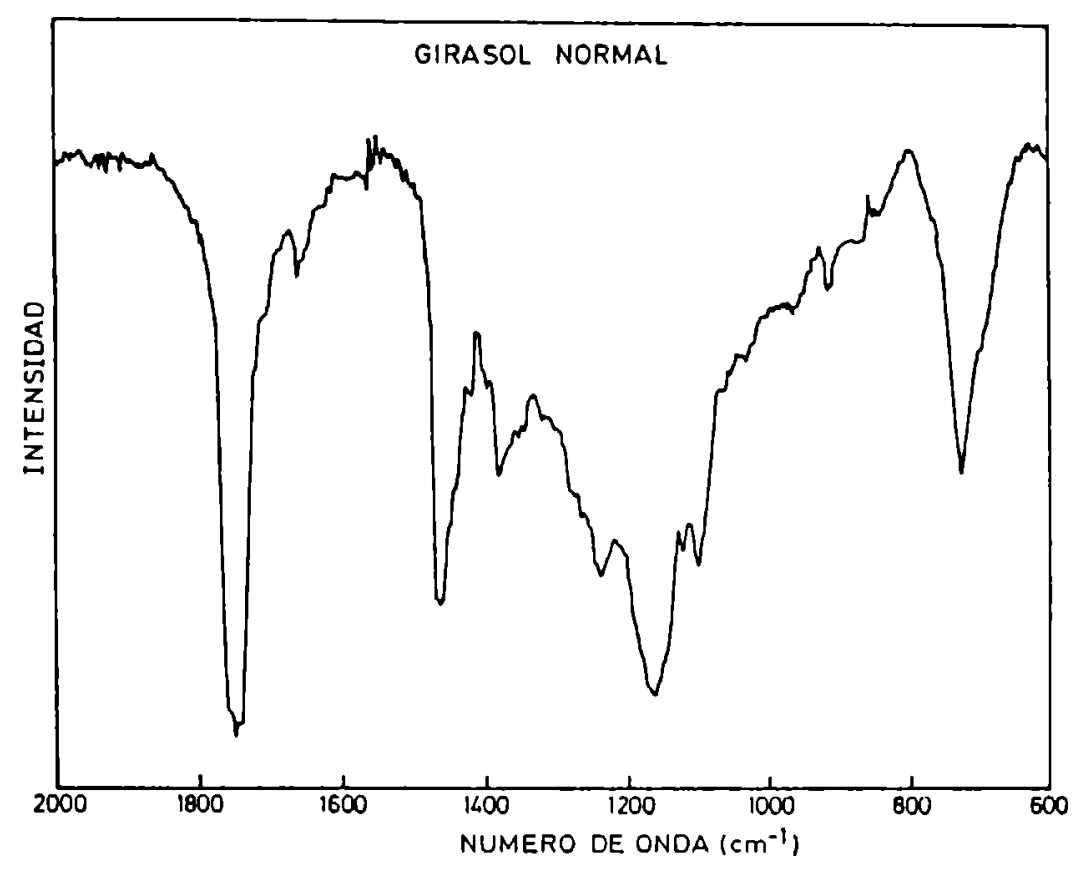

(a)

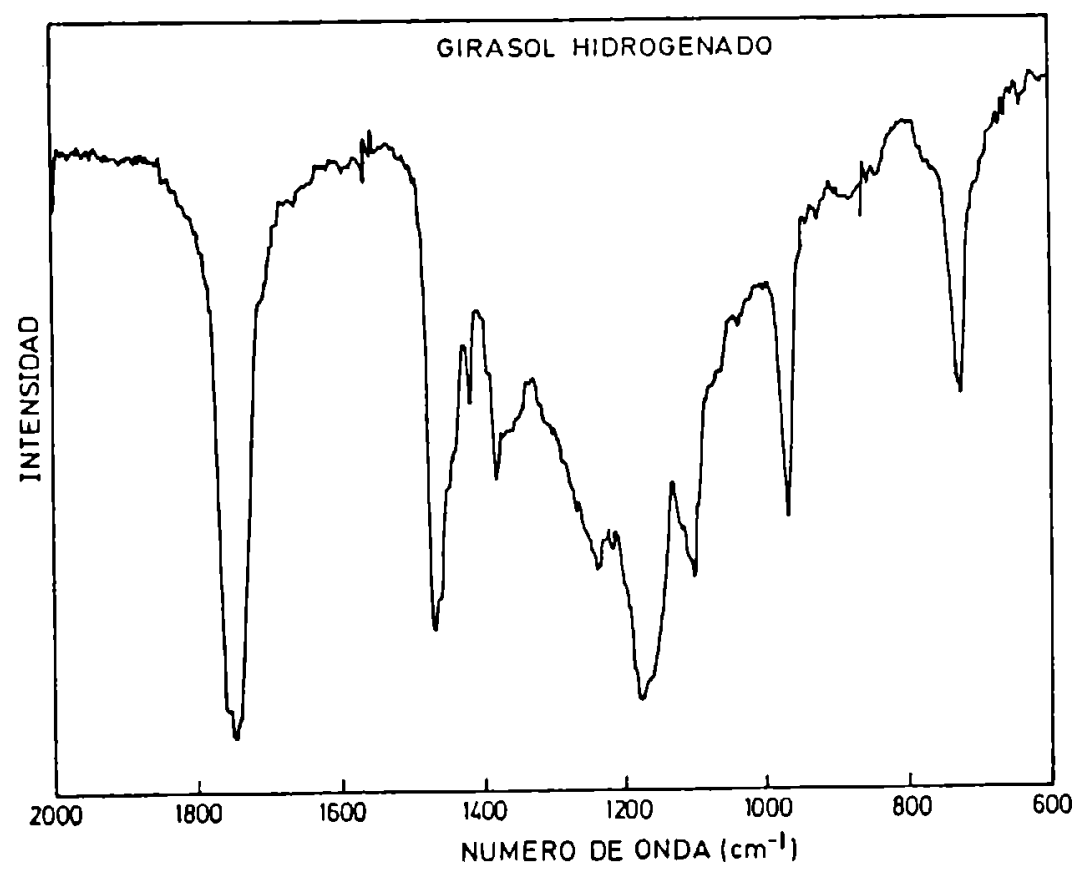

(b)

Figura 11. Espectros infrarrojos del aceite de girasol nornal (a) y aceite de girasol hidrogenado (b) 


\section{2 Caracterización de las fracciones solidas.}

Al igual que en el caso de las muestras originales las distintas fracciones sólidas obtenidas en las experiencias de fraccionamiento realizadas se analizaron en términos de su composición guimica (HPLC, GLC, IR y RHN) y de su estructura cristalina (DSC Y Rayos $X$ ).

2.2.1 Determinación cuantitativa de ácidos grasos por cromatoGrafia gaseosa (GLC).

Las Figuras 12 y 13 muestran los porcentajes de cada ácido graso en furción de la temperatura de cristalización. El análisis de las mismas revela que las fracciones que cristalizan a temperaturas superiores poseen un menor contenido de ácido linoleico y un mayor contenido de ácido estérico y oleico mientras que las que cristalizan por debajo de $33^{\circ} \mathrm{C}$ muestran un aumento en el contenido de ácido linoleico y una disminución en el contenido de ácido oleico. El ácido estéarico disminuye ligeramente al disminuir la temperatura. No se observaron diferencias significativas en el contenido de ácido palmitico. En general las muestras cristalizadas en forma dinámica en la primera experiencia presentaron un contenido de ácido palmitico ligeramente superior al de la muestra original. 


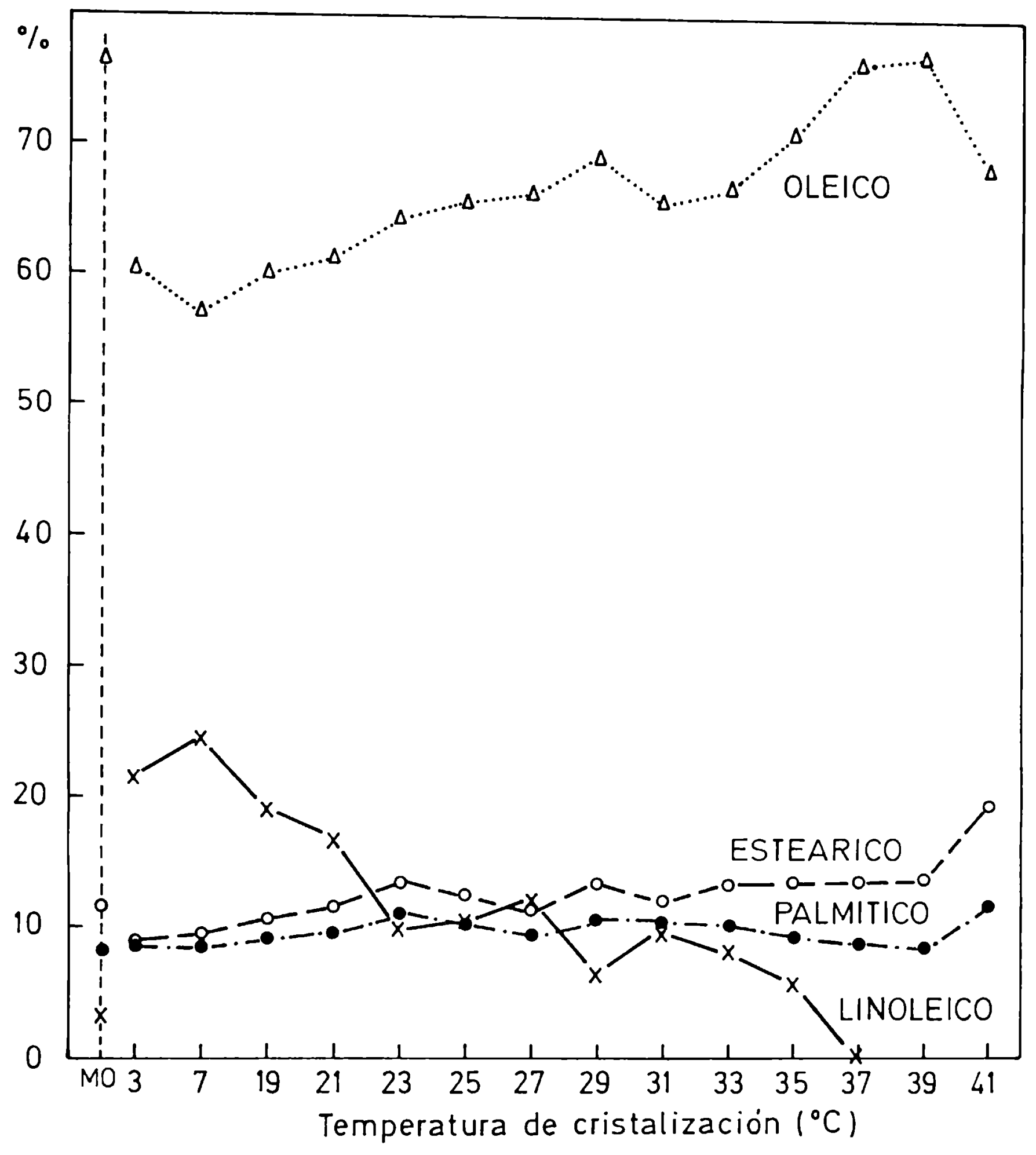

Figura 12. Porcentaje de los principales ácidos grasos detectados en función de la temperatura de cristalización (Fracciones sólidas, prinera experiencia) 


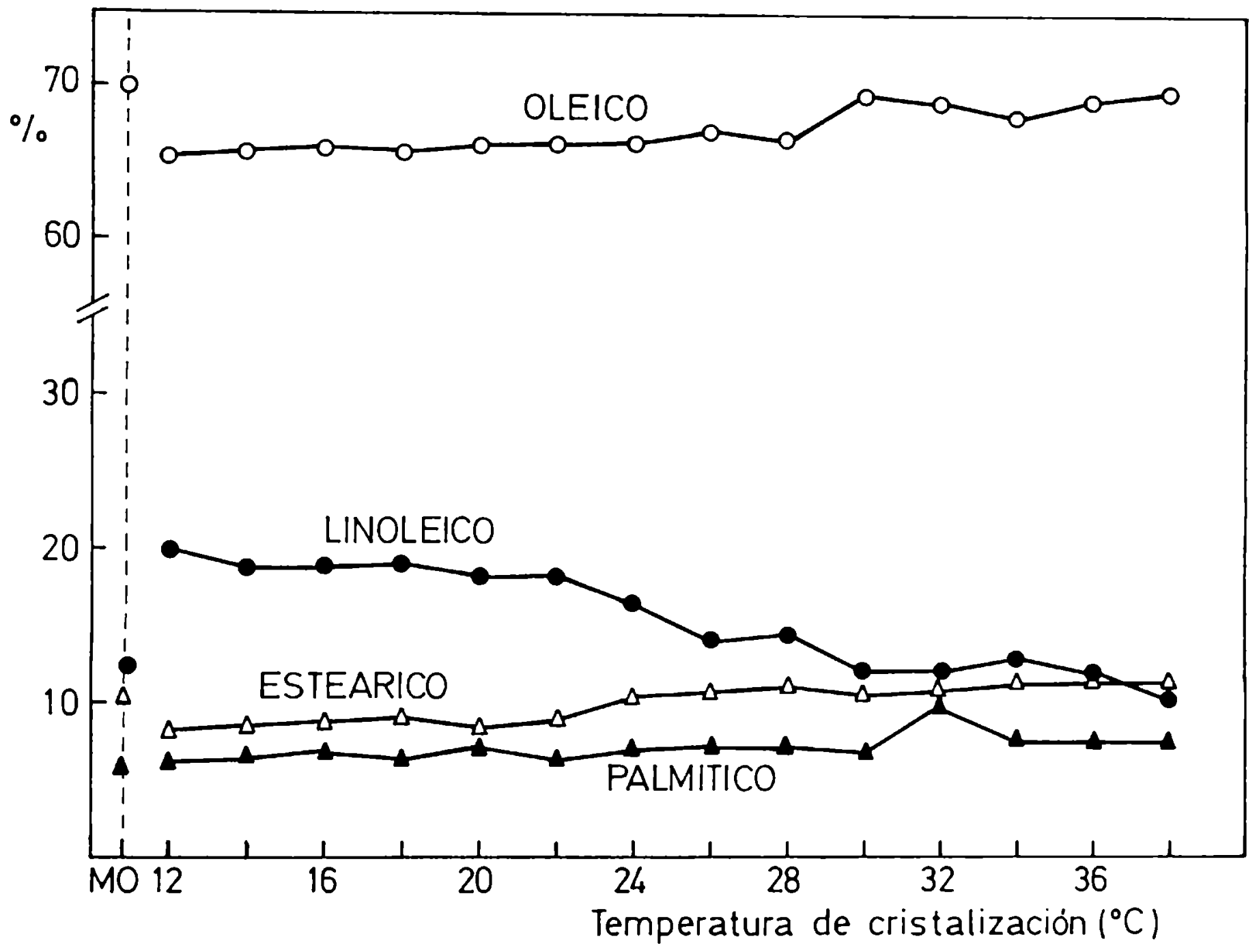

Figura 13. Porcentaje de los principales ácidos grasos detectados en función de la temperatura de cristalización (Fracciones sólidas, segunda experiencia) 
2.2.2 Determinación cuantitativa de triglicéridos por cronatografia liquida de alta presión.

La cromatografia liquida de alta presión permite separar triglicéridos por su tamaño molecular es decir por los diferentes largos en las cadenas sustituyentes en el glicerol y por el número de insaturaciones que presentan estos sustituyentes. Con este método como se ha mencionado anteriormente no es posible separar isómeros cis-trans a menos que se utilicen técnicas combinadas con ésta como preparación de derivados de los isómeros cis y trans que presentan diferentes tiempos de retención cuando son analizados en el cromatógrafo liquido (Wood y Lee, 1983). En las Tablas 6 y 7 se resumen los resultados que arrojaron las muestras sólidas obtenidas por fraccionamiento por temperatura de aceite de girasol hidrogenado. 
Tabla 6

Cromatografia liquida de alta presión correspondiente a la priner experiencia de fraccionaniento

Temp

Tiempos de retención (min).

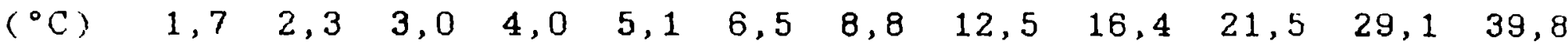

$41 \quad \cdots \quad--\quad 1,2<1,0 \quad 1,0 \quad 3,6 \quad 1,2 \quad 1,2 \quad 5,1 \quad 17,0 \quad 47,1 \quad 23,2$ $39 \quad \ldots<1,0<1,0 \quad 1,2<1,0 \quad 1,3 \quad 1,4 \quad 3,7 \quad 3 \quad 7,6 \quad 24,9 \quad 47,9 \quad 10,4$

$37 \ldots<1,0<1,0<1,0<1,0 \quad 1,0 \quad 1,5 \quad 2,3 \quad 6,3 \quad 25,3 \quad 52,8 \quad 8,4$ $35 \ldots<1,0 \ldots \ldots-2,8 \quad \ldots \quad 2,2 \quad 1,9 \quad 9,0 \quad 20,6 \quad 21,7 \quad 33,3 \quad 8,2$ $33 \quad 1,5<1,0 \quad \ldots \quad 5,9 \quad \ldots \quad 7,7 \quad 4,2 \quad 9,1 \quad 21,4 \quad 18,1 \quad 31,5$ $31 \quad 1,6<1,0 \quad \ldots \quad 5,1 \quad \ldots-10,5 \quad 5,5 \quad 7,4 \quad 22,6 \quad 12,3 \quad 34,7$ $29 \quad 3,6 \quad-\quad-\quad 5,6 \quad--10,7 \quad 7,8 \quad 7,2 \quad 24,0 \quad 11,1 \quad 30,0$

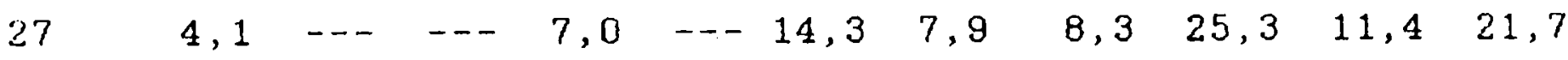
$25 \quad 5,1 \quad 1,3 \quad \ldots \quad 9,1 \quad \ldots \quad 11,9 \quad 8,2 \quad 8,2 \quad 26,0 \quad 11,5 \quad 18,7$ $23 \quad 2,2 \quad 2,9 \quad \ldots \quad 7,0 \quad \ldots-10,5 \quad 3,5 \quad 20,5 \quad 26,3 \quad 13,6 \quad 13,2$ $212,3<1,0 \quad \ldots \quad 9,0 \quad \ldots-10,7 \quad 4,8 \quad 18,9 \quad 27,3 \quad 12,4 \quad 13,9$ $192,0<1,0 \quad \ldots \quad 8,0 \quad \ldots \quad 9,0 \quad 5,5 \quad 20,0 \quad 27,9 \quad 12,7 \quad 14,5$ $7 \quad 1,3<1,0 \quad \ldots \quad 9,3 \quad \ldots \quad 9,1 \quad 4,5 \quad 20,6 \quad 28,9 \quad 13,7 \quad 12,2$ $3 \quad 1,2<1,0 \quad \ldots \quad 9,3 \quad \ldots \quad 9,3 \quad 2,3 \quad 24,7 \quad 28,1 \quad 12,4 \quad 12,4$ MO $\quad 1,0<1,0<1,0<1,0<1,0<1,0 \quad 2,0 \quad 4,2 \quad 7,3 \quad 25,5 \quad 48,4 \quad 12,0$

MO muestra original 
Tabla 7

Cronatografia liquida de las fracciones sólidas correspondientes a la segunda experiencia de fraccionamiento

\begin{tabular}{|c|c|c|c|c|c|c|c|c|c|c|c|c|}
\hline Temp & & & & & Tieme & pos de & rete & neión & $(\min )$ & & & \\
\hline$\left({ }^{\circ} \mathrm{C}\right)$ & 1,7 & 2,3 & 3,0 & 4,0 & 5,1 & 6,5 & 8,8 & 12,5 & 16,4 & 21,5 & 29,1 & 39,8 \\
\hline 38 & -- & --- & $<1,0$ & --- & $<1,0$ & $<1,0$ & 2,7 & 2,2 & 19,2 & 25,1 & 35,3 & 13,2 \\
\hline 36 & --- & $\ldots$ & $<1,0$ & $<1,0$ & $<1,0$ & 1,1 & 2,4 & 10,6 & 24,2 & 32,6 & 22,1 & 5,7 \\
\hline 34 & --- & $<1,0$ & $<1,0$ & $<1,0$ & $<1,0$ & 2,1 & 2,6 & 16,4 & 26,6 & 29,4 & 17,5 & 3,9 \\
\hline 32 & --- & $<1,0$ & $<1,0$ & --- & $<1,0$ & 1,1 & 2,1 & 16,3 & 34,8 & 29,8 & 14,7 & -- \\
\hline 30 & $<1,0$ & $<1,0$ & $<1,0$ & --- & 1,7 & 1,6 & 2,8 & 12,8 & 36,3 & 32,4 & 11,5 & --- \\
\hline 28 & $<1,0$ & $<1,0$ & $<1,0$ & $<1,0$ & 1,6 & 1,6 & 5,8 & 18,6 & 35,6 & 27,6 & 7,8 & --- \\
\hline 26 & $<1,0$ & $<1,0$ & $<1,0$ & $<1,0$ & 1,7 & 1,6 & 8,4 & 19,1 & 34,8 & 26,0 & 7,0 & --- \\
\hline 24 & $<1,0$ & $<1,0$ & 4,8 & 0,6 & 1,5 & 1,5 & 9,3 & 18,5 & 34,5 & 21,8 & 7,0 & --- \\
\hline 22 & $<1,0$ & $<1,0$ & 2,7 & 0,6 & 1,2 & 1,2 & 13,4 & 20,6 & 31,4 & 22,3 & 5,8 & -- \\
\hline 20 & $<1,0$ & $<1,0$ & 2,4 & $<1,0$ & 1,2 & 1,1 & 11,1 & 21,7 & 32,4 & 23,0 & 6,0 & --- \\
\hline 18 & $<1,0$ & 2,4 & $<1,0$ & 1,4 & 1,2 & 1,2 & 10,1 & 18,8 & 34,1 & 23,4 & 6,1 & --- \\
\hline 16 & $<1,0$ & $<1,0$ & 4,2 & $<1,0$ & 1,8 & 1,8 & 14,2 & 25,5 & 29,4 & 16,5 & 4,6 & --- \\
\hline 14 & $<1,0$ & $<1,0$ & 5,6 & 1,2 & 1,3 & 1,5 & 15,2 & 25,3 & 28,3 & 15,8 & 4,9 & -- \\
\hline 12 & $<1,0$ & $<1,0$ & $<1,0$ & 2,1 & 2,1 & 1,5 & 22,0 & 30,5 & 23,8 & 14,5 & 2,6 & --- \\
\hline MO & $<1,0$ & $<1,0$ & $<1,0$ & $<1,0$ & 1,1 & $<1,0$ & 2,8 & 18,1 & 39,5 & 29,4 & 7,0 & 0,8 \\
\hline
\end{tabular}

MO muestra origina.1 
Para facilitar el analisis de los resultados se graficó sólo para los componentes mayoritarios el porcentaje de cada uno de ellos en función de sus respectivos tiempos de retención (Figuras 14 y 15) y los mismos porcentejes en función de la temperatura de cristalización (Figuras 17 y 18) para la primera y segunda experiencia respectivamente. Para construir las Figuras 14 y 15 se ha elegido una temperatura de cristalización alta, una media y una baja. (Experiencia 1, fracciones $41^{\circ} \mathrm{C}, 31^{\circ} \mathrm{C}$ y $21^{\circ} \mathrm{C}$; Experiencia 2 , fracciones $38^{\circ} \mathrm{C}, 24^{\circ} \mathrm{C}$ y $14^{\circ} \mathrm{C}$ )

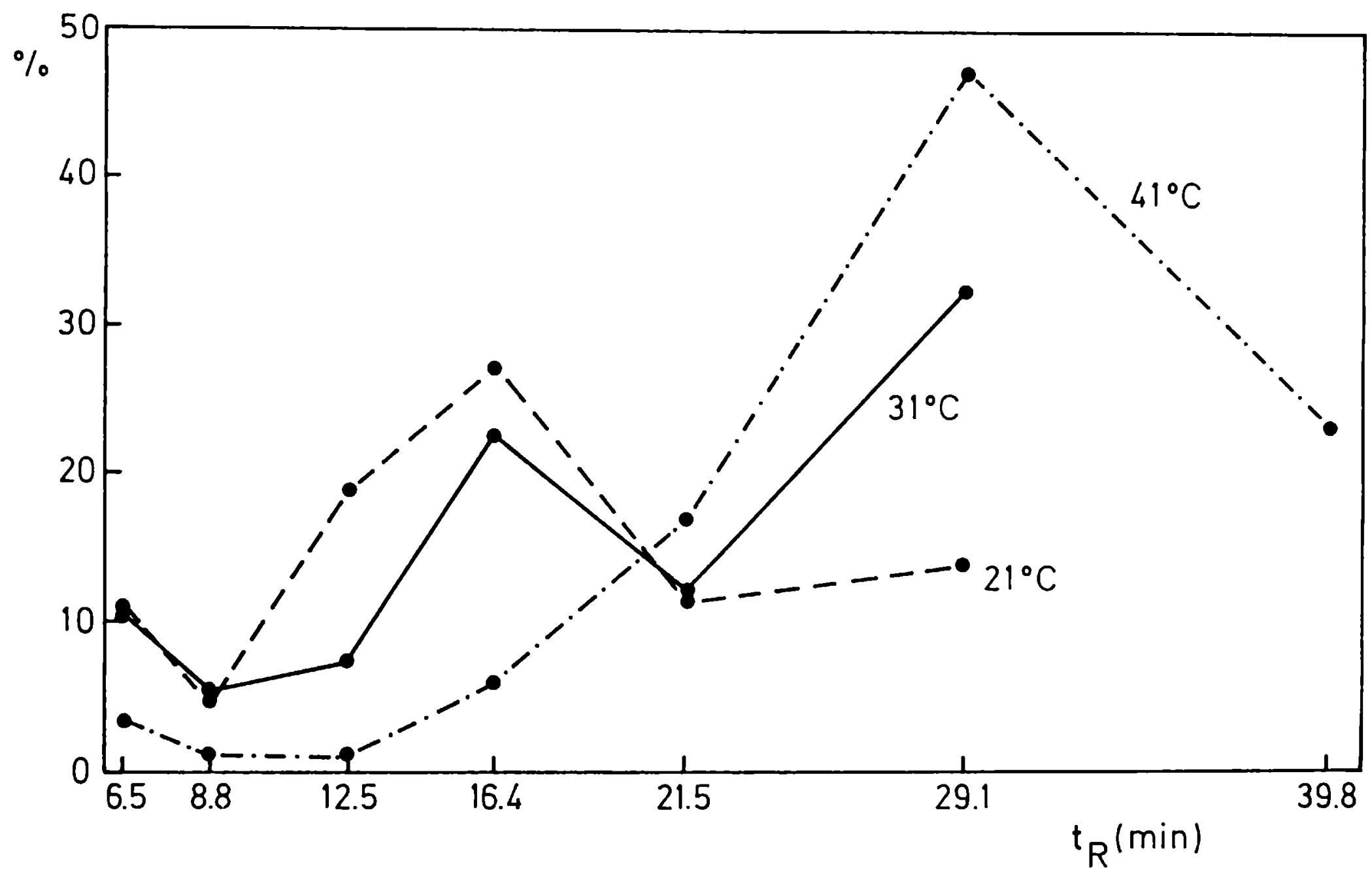

Figura 14. Porcentaje de los triglicéridos mayoritarios en función de sus tienpos de retención para las fracciones $41^{\circ} \mathrm{C}, 31^{\circ} \mathrm{C}$ y $21^{\circ} \mathrm{C}$ (Prinera experiencia) 


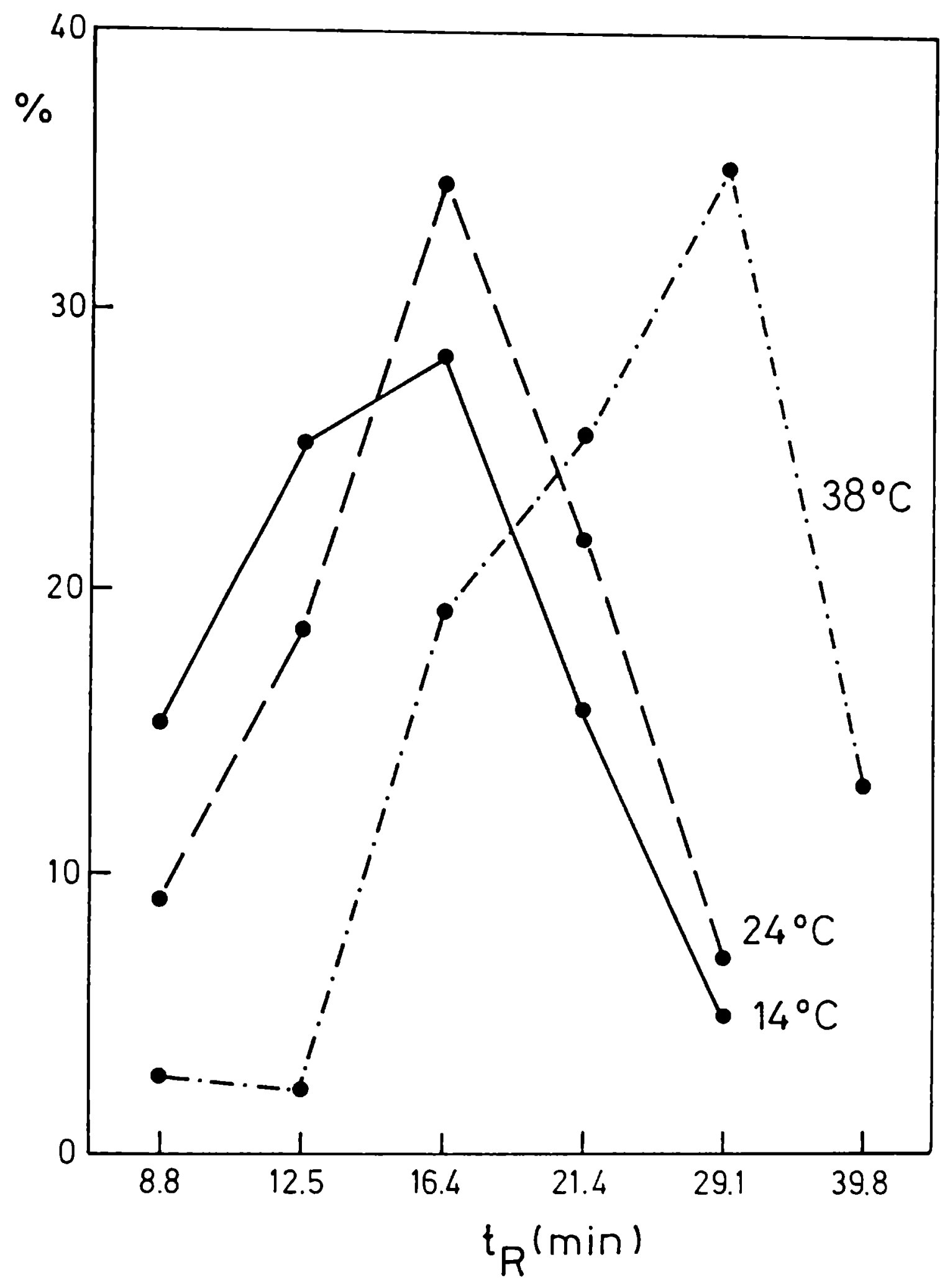

Figura 15. Porcentaje de los triglicéridos nayoritarios en función de sus tiempos de retención para las fracciones $38^{\circ} \mathrm{C}, 24^{\circ} \mathrm{C}$ y $14^{\circ} \mathrm{C}$ (Segunda experiencia) 


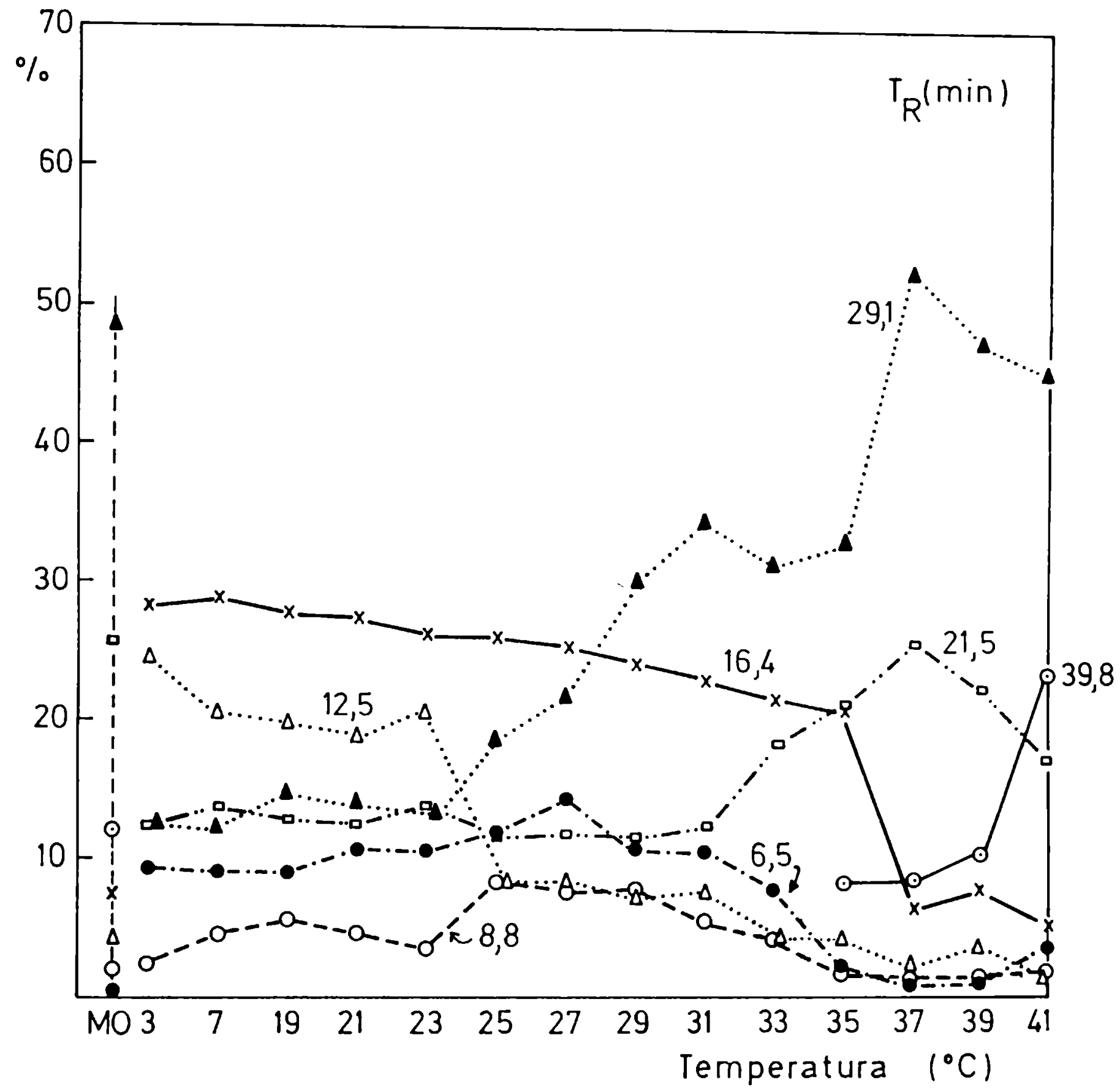

Figura 16. Porcentaje de los triglicéridos mayoritarios en función de la temperatura de cristalizacion. Primera experiencia. 


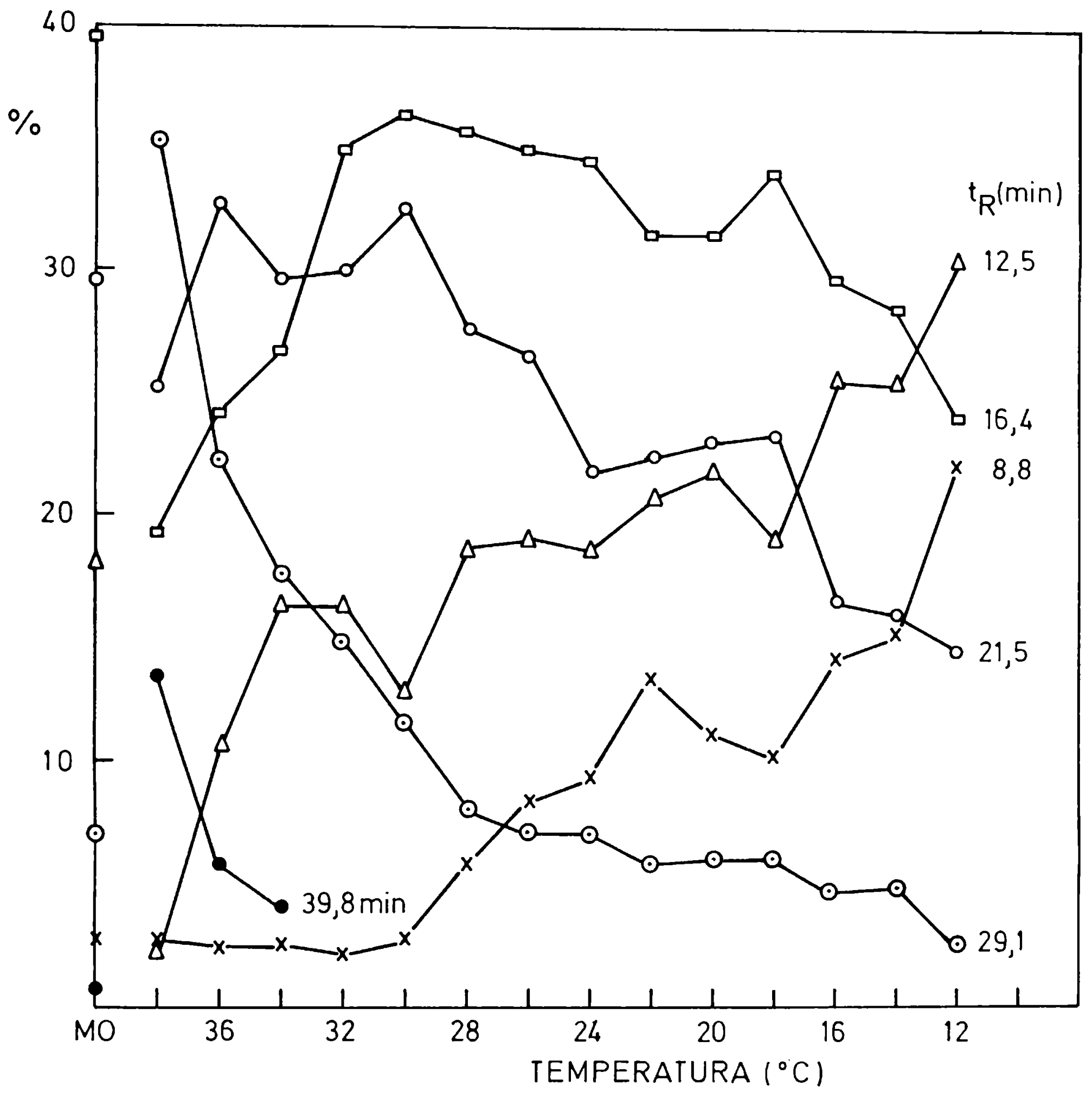

Figura 17. Porcentaje de los triglicéridos mayoritarios en función de la temperatura de cristalización. Segunda Experiencia 
Los resultados obtenidos al analizar los aceites vegetales de composición conocida on trigliceridos nos ha permitido asignar tentativamente como componente mayoritario de cada uno de los picos encontrados en los cromatogramas, correspondientes a las muestras obtenidas por cristalización fraccionada, los que figuran a continuación. Sin embargo no se puede descartar la posibilidad que en cada pico esten presentes uno o más triglicéridos de ECNs equivalentes en cantidades minoritarias.

SSO: en la primera experiencia este triglicérido representó un $23,2 \%$ en la muestra cristalizada a $41^{\circ} \mathrm{C}$ y porcentejes del orden de $8 \%$ a $10 \%$ en las muestras cristalizadas a $39^{\circ} \mathrm{C}, 37^{\circ} \mathrm{C}$ y $35^{\circ} \mathrm{C}$ no detectandose a temperaturas inferiores; en la segunda experiencia en cambio en la única fracción en la que resultó importante fue en la extraida a $38^{\circ} \mathrm{C}$. En las fracciones cristalizadas a $36^{\circ} \mathrm{C}$ y $34^{\circ} \mathrm{C}$ se encuentró en el orden de $6 \%$ y $4 \%$ respectivamente no hallándose en el resto.

S00: en la primera experiencia se encontró alrededor de un 50\% en las muestras recogidas a $41^{\circ} \mathrm{C}, 39^{\circ} \mathrm{C}$ y $37^{\circ} \mathrm{C}$ y fue descendiendo con la temperatura hasta un valor cercano a $15 \%$. En la segunda experiencia resultó el componente mayoritario a $38^{\circ} \mathrm{C}$ y representó porcentajes superiores a la muestra original hasta $30^{\circ} \mathrm{C}$. En el resto de las fracciones se encuentra en un porcentaje menor el cual disminuye al descender la temperatura de cristalización.

P0O: en la primera experiencia en las fracciones cristalizadas a $39^{\circ} \mathrm{C}, \quad 37^{\circ} \mathrm{C}$ y $35^{\circ} \mathrm{C}$ se registraron valores del orden de la muestra 
original. A todas las otras temperaturas se encontraron valores inferiores. En las muestras recogidas en la segunda experiencia se halló en porcentajes altos en todas las fracciones; por encina de $28^{\circ} \mathrm{C}$ con un contenido similar al de la muestra original y por debajo disminuyó hasta valores de $15 \%$.

000: $\in$ n la primera experiencia de fraccionamiento se encontró que las muestras cristalizadas a $41^{\circ} \mathrm{C}, 39^{\circ} \mathrm{C}$ y $37^{\circ} \mathrm{C}$ presentaron contenidos del orden de la muestra original mientras que en las restantes fracciones el contenido aumentó al descender la temperatura. En la segunda experiencia los contenidos de todas las fracciones resultaron inferiores al de la muestra original, disminuyendo al descender la temperatura alcanzando valores cercanos a $24 \%$ a $12^{\circ} \mathrm{C}$.

LOO: en la primera experiencia de fraccionamiento este triglicérido representó en las fracciones superiores porcentajes menores del 10\%; al disminuir la temperatura de cristalización fue aumentando hasta alcanzar valores del $20 \%$ a $19^{\circ} \mathrm{C}$. En la segunda experiencia se presentó en valores menores a los encontrados en la muestra original hasta $28^{\circ} \mathrm{C}$ y aumentó luego al disminuir la temperatura de cristalización, alcanzando valores del $30 \%$ a $12^{\circ} \mathrm{C}$.

LLP: en la primera experiencia en todas las fracciones se halló en porcentajes menores al 10\%. En la segunda experiencia se registraron en cambio valores superiores al de la muestra original a partir de $28^{\circ} \mathrm{C}$ y fue aumentando al descender la temperatura hasta alcanzar $22 \%$ en la fracción $12^{\circ} \mathrm{C}$. 
Otros triglicéridos probablemente formados por ácidos grasos de cadenas más cortas o más insaturados aparecen a tiempos de retención menores pero no son importantes.

En la Figura 18 se comparan tres cromatogramas pertenecientes a muestras cristalizadas a $37^{\circ} \mathrm{C}, 29^{\circ} \mathrm{C}$ y $19^{\circ} \mathrm{C}$ colectadas on la primer experiencia de fraccionamiento con la muestra original. De acuerdo con los perfiles y composición quimica obtenidos podemos dividir a las muestras en tres grupos: un primer grupo que comprende las fracciones de temperatura de cristalización entre $31^{\circ} \mathrm{C}$ y $41^{\circ} \mathrm{C}$, un segundo grupo cristalizadas entre $23^{\circ} \mathrm{C}$ y $31^{\circ} \mathrm{C}$ y un tercer grupo entre $12^{\circ} \mathrm{C}$ y $23^{\circ} \mathrm{C}$. En la Figura 18 se ha incluido un cromatograma de cada grupo. Las muestras correspondientes a las fracciones $7^{\circ} \mathrm{C}$ y $3^{\circ} \mathrm{C}$ también fueron analizadas y de acuerdo con los resultados encontrados pueden considerarse como pertenecientes al tercer grupo. Es interesante destacar que estos tres grupos son coincidentes con los tres señalados al analizar el aumento del contenido de sólidos en cada fracción (Figura 4). El análisis de la Figura 18 arroja como conclusión que los cortes obtenidos a mayor temperatura de fraccionamiento se enriquecen en triglicéridos de número equivalente de carbonos altos. En los cortes de menor temperatura de fraccionamiento son importantes los triglicéridos de ECN menor y las fracciones intermedias se enriquecen en los triglicéridos de tiempos de retención medio. Esto se pone claramente de manifiesto en las Figuras 15 y 16. 


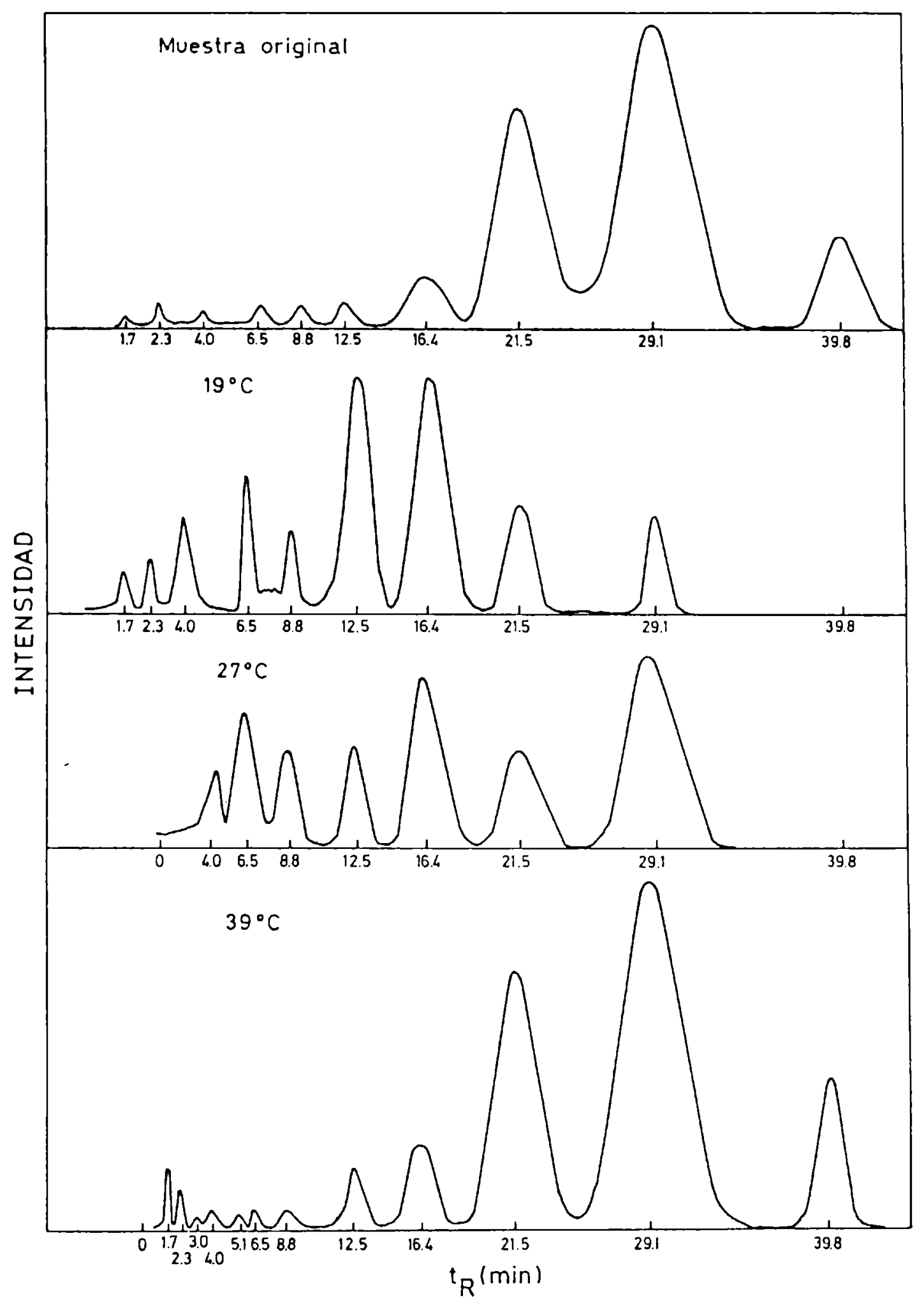

Figura 18. Cronatogramas de las muestras cristalizadas a $37^{\circ} \mathrm{C}$, $29^{\circ} \mathrm{C}$ y $19^{\circ} \mathrm{C}$ y el correspondiente a la muestra original (Prinera experiencia) 


\subsubsection{Resonancia magnética nuclear}

Para verificar la composición quimica de las fracciones y la muestra original se realizaron estudios de resonancia magnética nuclear. En la Tabla 8 se resumen los resultados hallados tomando a modo de ejemplo una frucción de cada grupo según su composición en triglicéridos y la muestra original 2. Las fracciones de la primera experiencia asi como la muestra original arrojaron resultados concordantes con los que se resumen a continuación.

Tabla 8

Resonancia magnética nuclear de las fracciones y la nuestra original

\begin{tabular}{|ccccc|}
\hline Muestra & $\begin{array}{l}\text { Número de } \\
\text { protones } \\
\text { olefinicos }\end{array}$ & $\begin{array}{l}\text { Numero de } \\
\text { protones } \\
\text { totales }\end{array}$ & $\begin{array}{c}\text { PM } \\
\text { medio }\end{array}$ & $\begin{array}{c}\text { Indice } \\
\text { de iodo }\end{array}$ \\
\hline $38^{\circ} \mathrm{C}$ & 4 & 86 & 748,4 & 86 \\
$24^{\circ} \mathrm{C}$ & 4 & 82 & 710,0 & 78 \\
$14^{\circ} \mathrm{C}$ & 6 & 80 & 704,0 & 97 \\
MO & 4 & 86 & 740,0 & 68 \\
\hline
\end{tabular}

MO nuestra original 
De acuerdo a los datos obtenidos 18 muestra original posee una composición semejante a las fracciones superiores, mientras gue la muestra recogida a $14^{\circ} \mathrm{C}$ es más insaturada gue las extraldas del filtro a $24^{\circ} \mathrm{C}$ y $38^{\circ} \mathrm{C}$. Esto coincide con su mayor contenido en LOO y su menor contenido en SSO y SDO.

\subsubsection{Calorinetria diferencial de barrido}

Todas las muestras obtenidas en estas experiencias fueron analizadas por calorimetria diferencial de barrido. Con el fin de presentar los resultados en forma clara se ha tomado como ejemplo una temperatura perteneciente a cada uno de los grupos diferenciados por su composición en triglicéridos. La Figura 19 muestra los termogramas de las muestras sólidas recogidas a $41^{\circ} \mathrm{C}, \quad 31^{\circ} \mathrm{C}$ y $21^{\circ} \mathrm{C}$ en la primer experiencia y la Figura 20 los correspondientes a $38^{\circ} \mathrm{C}, 24^{\circ} \mathrm{C}$ y $14^{\circ} \mathrm{C}$ de la segunda experiencia. Todos los perfiles de las fracciones en ambas experiencias resultaron diferentes a los de la muestra original. Las muestras obtenidas a $38^{\circ} \mathrm{C}$ y $41^{\circ} \mathrm{C}$ tienen componentes con puntos de fusión mayores que las otras muestras. Al descender la temperatura de fraccionamiento los diagramas se van angostando. Las áreas de los picos de temperaturas más elevadas son mayores en las muestras del primer grupo y van disminuyendo al descender la temperatura. Esto coincide con el hecho que la composición en trigliceridos del primer grupo es rica en SOO y POO, la del segundo grupo en trigliceridos de tiempos de retención intermedios y las del tercer grupo en LOO. 


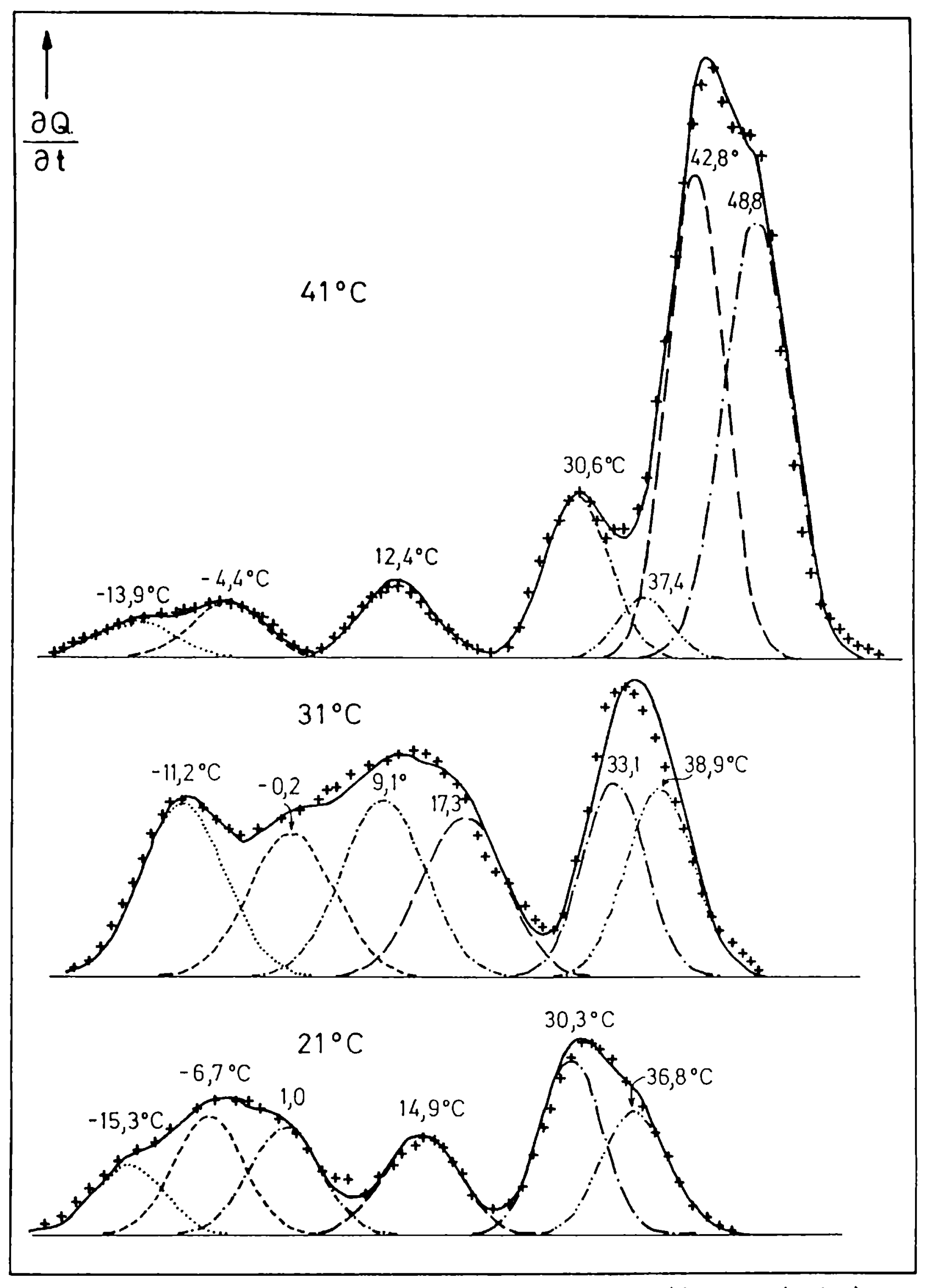

tiempo $(\min )$

Figura 19. Ternogranas interpretados natenáticanente correspondientes a las fracciones sólidas $41^{\circ} \mathrm{C}, 31^{\circ} \mathrm{C}$ y $21^{\circ} \mathrm{C}$ (Primera experiencia) 


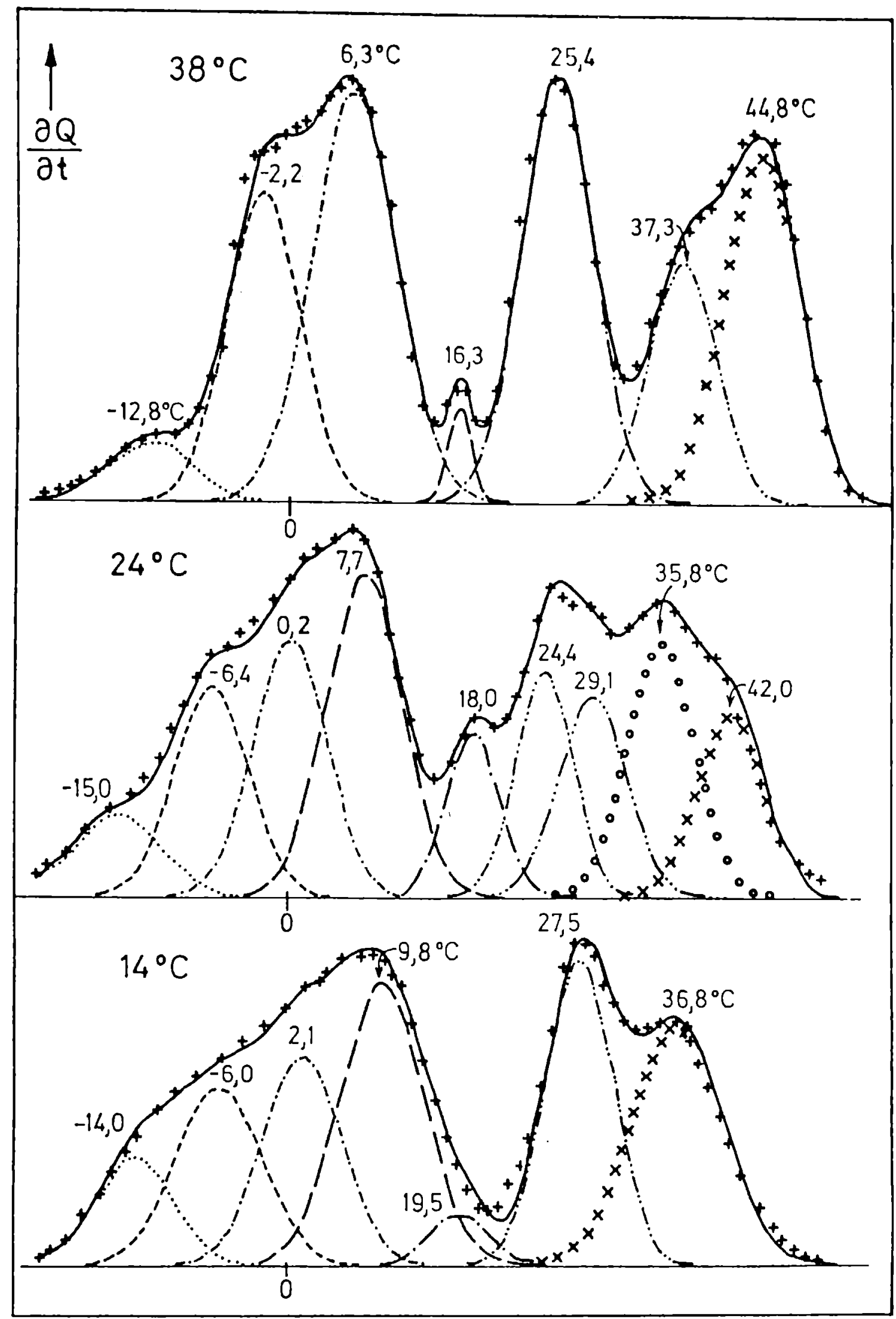

tiempo (min)

Pigura 20. Ternogramas interpretados natenáticamente correspondientes a las fracciones sólidas $38^{\circ} \mathrm{C}, 24^{\circ} \mathrm{C}$ y $14^{\circ} \mathrm{C}$ (Segunda experiencia) 
Los termogramas fueron interpretados aplicando el modelo matemático citado anteriormente. En la primera experiencia la fracción extraida del filtro a $41^{\circ} \mathrm{C}$ presentó siete picos cuyas temperaturas fueron $-13,9^{\circ} \mathrm{C} ;-4,4^{\circ} \mathrm{C} ; 12,4^{\circ} \mathrm{C} ; 30,6^{\circ} \mathrm{C} ; 37,4^{\circ} \mathrm{C}$; $42,8^{\circ} \mathrm{C}$ y $48,8^{\circ} \mathrm{C}$ en porcentajes iguales a 3,$5 ; 5,1 ; 6,4 ; 12,6 ; 4,5 ;$ 28,4 y 39,4 respectivamente. La fracción $31^{\circ} \mathrm{C}$ pudo ser interpretada mediante la suma de seis componentes de temperaturas de pico $-11,2^{\circ} \mathrm{C} ; \quad-0,2^{\circ} \mathrm{C} ; \quad 9,1^{\circ} \mathrm{C} ; \quad 17,3^{\circ} \mathrm{C} ; \quad 33,1^{\circ} \mathrm{C}$ y $38,9^{\circ} \mathrm{C}$ y porcentajes 17,$9 ; 15,4 ; 19,1 ; 16,4 ; 20,4$ y 10,7 . El termograma perteneciente a la fracción cristalizada a $21^{\circ} \mathrm{C}$ sugirió la presencia de seis picos de temperatura $-15,3^{\circ} \mathrm{C} ;-6,7^{\circ} \mathrm{C} ; 1,0^{\circ} \mathrm{C} ; 14,9^{\circ} \mathrm{C} ; \quad 30,3^{\circ} \mathrm{C}$ y $36,8^{\circ} \mathrm{C}$ y áreas iguales a $11,3 \% ; 18,1 \% ; 15,4 \% ; 21,8 \% ; 22,6 \%$ y $10,8 \%$. Los constituyentes de $48,8^{\circ} \mathrm{C}$ y $42,8^{\circ} \mathrm{C}$ se encontraron sólo en las fracciones del primer grupo mientras que no se hallaron presentes en los otros dos grupos. Por otra parte las fracciones inferiores presentaron constituyentes con puntos de fusión menores que $0^{\circ} \mathrm{C}$ en porcentajes importantes.

En la segunda experiencia la fracción cristalizada a $38^{\circ} \mathrm{C}$ se interpreto como formada por siete constituyentes de punto de fusión $-12,8 ;-2,2^{\circ} \mathrm{C} ; 6,3^{\circ} \mathrm{C} ; 16,3^{\circ} \mathrm{C} ; 25,4^{\circ} \mathrm{C} ; 37,3^{\circ} \mathrm{C}$ y $44,8^{\circ} \mathrm{C}$ y áreas porciento 3,$2 ; 16,8 ; 25,0 ; 1,5 ; 22,2 ; 11,6 ; y$ 19,7. La fracción $24^{\circ} \mathrm{C}$ presentó un perfil que sugirió 10 presencia de nueve componentes de temperatura de pico $-15,0^{\circ} \mathrm{C} ;-6,4^{\circ} \mathrm{C} ; 0,2^{\circ} \mathrm{C} ; 7,7^{\circ} \mathrm{C}$; $18,0^{\circ} \mathrm{C} ; \quad 24,4^{\circ} \mathrm{C} ; \quad 29,1^{\circ} \mathrm{C} ; \quad 35,8^{\circ} \mathrm{C}$ y $42,0^{\circ} \mathrm{C}$ y áreas porciento 6,$0 ;$ 11,$7 ; 14,4 ; 19,2 ; 6,3 ; 9,3 ; 11,1 ; 13,0$ y 8,0 . La fracción $14^{\circ} \mathrm{C}$ estaria formada por siete constituyentes de temperaturas de pico $-14,0^{\circ} \mathrm{C} ; \quad-6,0^{\circ} \mathrm{C} ; \quad 2,1^{\circ} \mathrm{C} ; \quad 9,8^{\circ} \mathrm{C} ; \quad 19,5^{\circ} \mathrm{C} ; 27,5^{\circ} \mathrm{C}$ y $36,8^{\circ} \mathrm{C}$ y áreas porciento 7,$4 ; 14,3 ; 16,2 ; 21,2 ; 2,0 ; 19,7$ y 19,2 . Las fracciones 
del primer grupo presentaron un pico a $44,9^{\circ} \mathrm{C}$ que resulto importante el que luego disminuyó en porcentaje al disminuir la temperatura no encontrándose por debajo de $24^{\circ} \mathrm{C}$. A medida gue la temperatura de cristalización fue descendiendo las fracciones presentaron menores contenidos de constituyentes con altos puntos de fusión y mayores cantidades de componentes insaturados.

Las entalpias de fusión de los constituyentes fueron calculadas según se explicó en Materiales y Kétodos (sección 5.5). No siempre las entalpias para una misma temperatura de pico resultaron del mismo orden dado que todas las muestras diferian en composición quimica y posiblemente las solubilidades entre los distintos triglicéridos se vieran modificadas debido a esta diferencia en la composición, es decir que cada constituyente podria resultar formado por diferentes triglicéridos y la mezcla poseer idéntica temperatura de fusión. El cálculo de las entalpias totales arrojó los siguientes resultados: las fracciones extraidas en la primera experiencia a $41^{\circ} \mathrm{C}, \quad 31^{\circ} \mathrm{C}$ y $21^{\circ} \mathrm{C}$ presentaron valores de 92,$9 ; 85,7$ y $67,1 \mathrm{Joule} / \mathrm{g}$ y 1 as obtenidas en 1 a segunda 82,$8 ; 76,2$ y $63,6 \mathrm{Jou} l \mathrm{e} / \mathrm{g}$. Las entalpias totales de las muestras descendieron al descender la temperatura de cristalización es decir que las muestras fraccionadas a menor temperatura se enriquecieron en triglicéridos insaturados los cuales poseen menores calores de fusión. La contribución a la entalpia total de los constituyentes de mayor punto de fusión fue superior en las fracciones de mayor temperatura de cristalización. 


\subsubsection{Difractonetria de rayos $\mathrm{X}$.}

Las fracciones sólidas cristalizadas a $21 / 14,31 / 24$ y $41 / 38^{\circ} \mathrm{C}$ fueron estudiadas por difractometria de rayos $X$ y comparadas con las muestras originales respectivas. En ambos casos las fracciones presentaron diferentes diagramas que las muestras originales. En la Figura 21 se muestran los diagramas correspondientes a las fracciones recogidas a $21^{\circ} \mathrm{C}, 31^{\circ} \mathrm{C}$ y $41^{\circ} \mathrm{C}$ (primera experiencia) y en la Figura 22 (a) los correspondientes a las muestras $14^{\circ} \mathrm{C}, 24^{\circ} \mathrm{C}$ y $38^{\circ} \mathrm{C}$ (segunda experiencia). Los diagramas correspondientes a las distintas fracciones fueron similares entre sí, presentando pequeñas diferencias en la importancia relativa de los picos. Las fracciones obtenidas a $21^{\circ} \mathrm{C}, \quad 31^{\circ} \mathrm{C}$ y $41^{\circ} \mathrm{C}$ presentaron cristales tipo $\beta^{\circ}$ asi tambien como las extraidas a $14^{\circ} \mathrm{C}, 24^{\circ} \mathrm{C}$ y $38^{\circ} \mathrm{C}$. Este tipo de cristales era esperable ya que su filtración durante las experiencias de cristalización resultó facil. La muestra obtenida a $14^{\circ} \mathrm{C}$ fue fundida y cristalizada en forma estática a esa temperatura, en este caso se formó una mezcla de cristales $\beta^{\circ}$ y $\beta$. En la Figura 22 (b) se presenta este diagrama. La diferencia entre una cristalización estática y una dinamica se pone aquí de manifiesto.

Cuando se intentó correlacionar los resultados hallados por cromatografia liquida de alta presión y por calorimetria diferencia). de barrido (Resultados y Discusión sección 2.1.3) se sugirió que fodian ocurrir cambios polimorficos durante la fusión de las mismas $y$ obtener asi un contenido de sólidos superior al real. Tanto los diagramas de rayos $\mathrm{X}$ de las muestras originales como los de las fracciones presentaron formas $\beta^{\prime} \circ$ mezclas $\beta^{\prime}-\beta$. Según 
estos diagramas no deberian esperarse transformaciones $\alpha \longrightarrow \beta^{\circ} \circ$ $\because->\beta^{\prime}$. Sin embargo debido a la existencia de liquido ocluido por los cristales de grasa es posible que al llevar las muestras a $-40.0^{\circ} \mathrm{C}$ (temperatura elegida como inicial en 108 termogramas) se formaran fases inestables que luego se transformaran en otras más estables. Para verificar esta suposición deberiamos tener la posibilidad de realizar diagramas de rayos $X$ siguiendo la misma historia térmica que la empleada para obtener los termogramas.

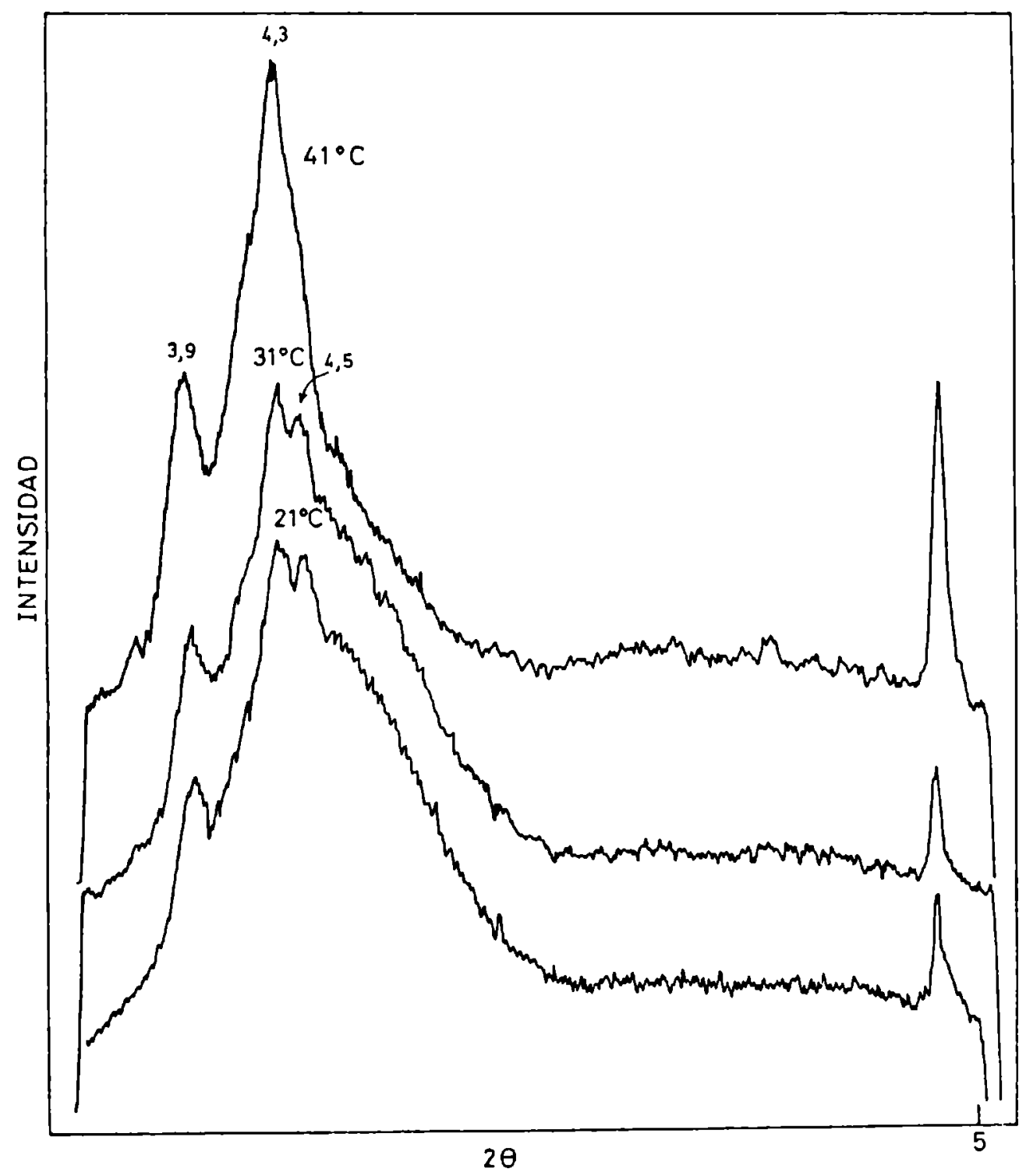

Figura 21. Espectros de rayos $X$ de las muestras cristalizadas a $21^{\circ} \mathrm{C}, 31^{\circ} \mathrm{C}$ y $41^{\circ} \mathrm{C}$ (Prinera experiencia). 


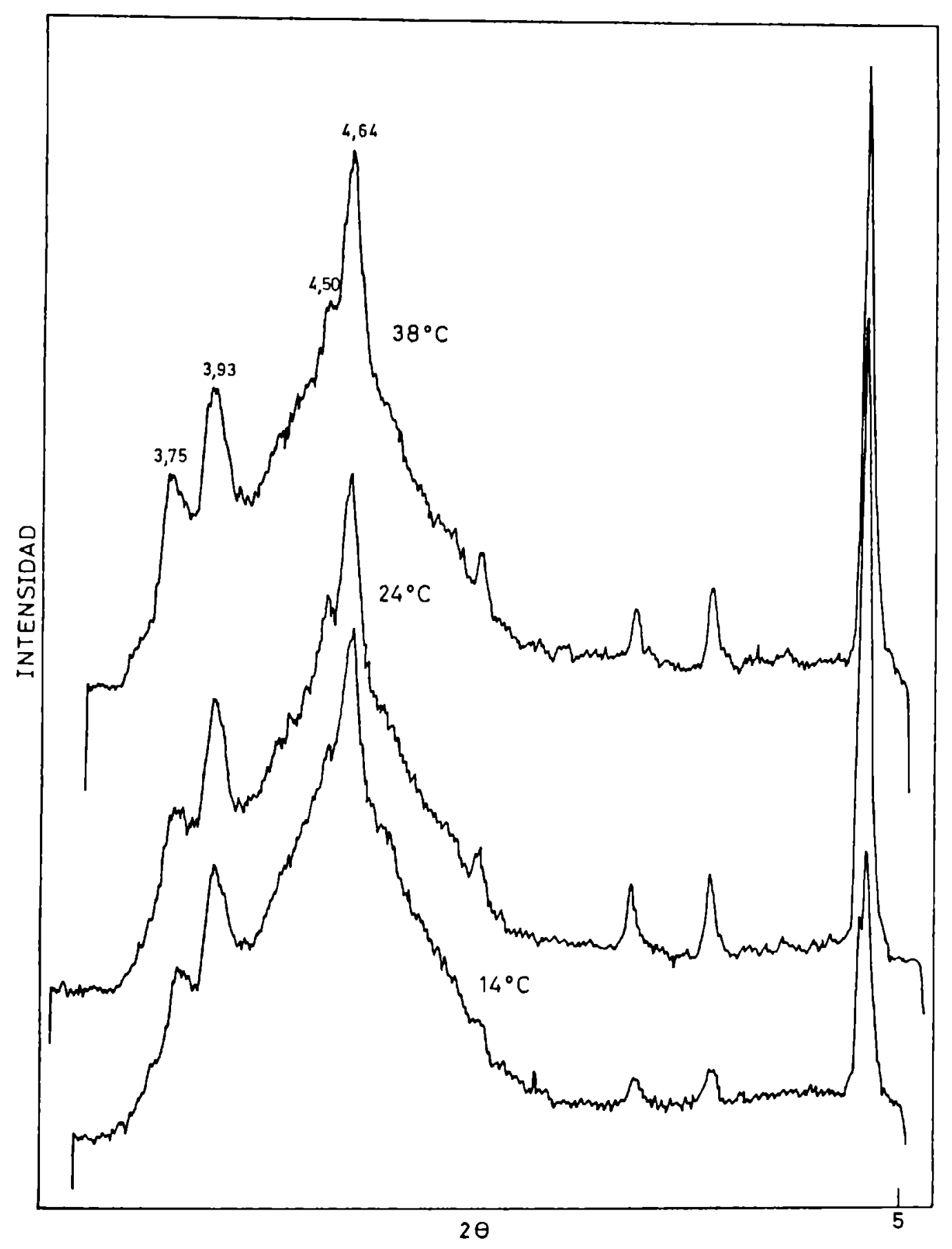

(a) 


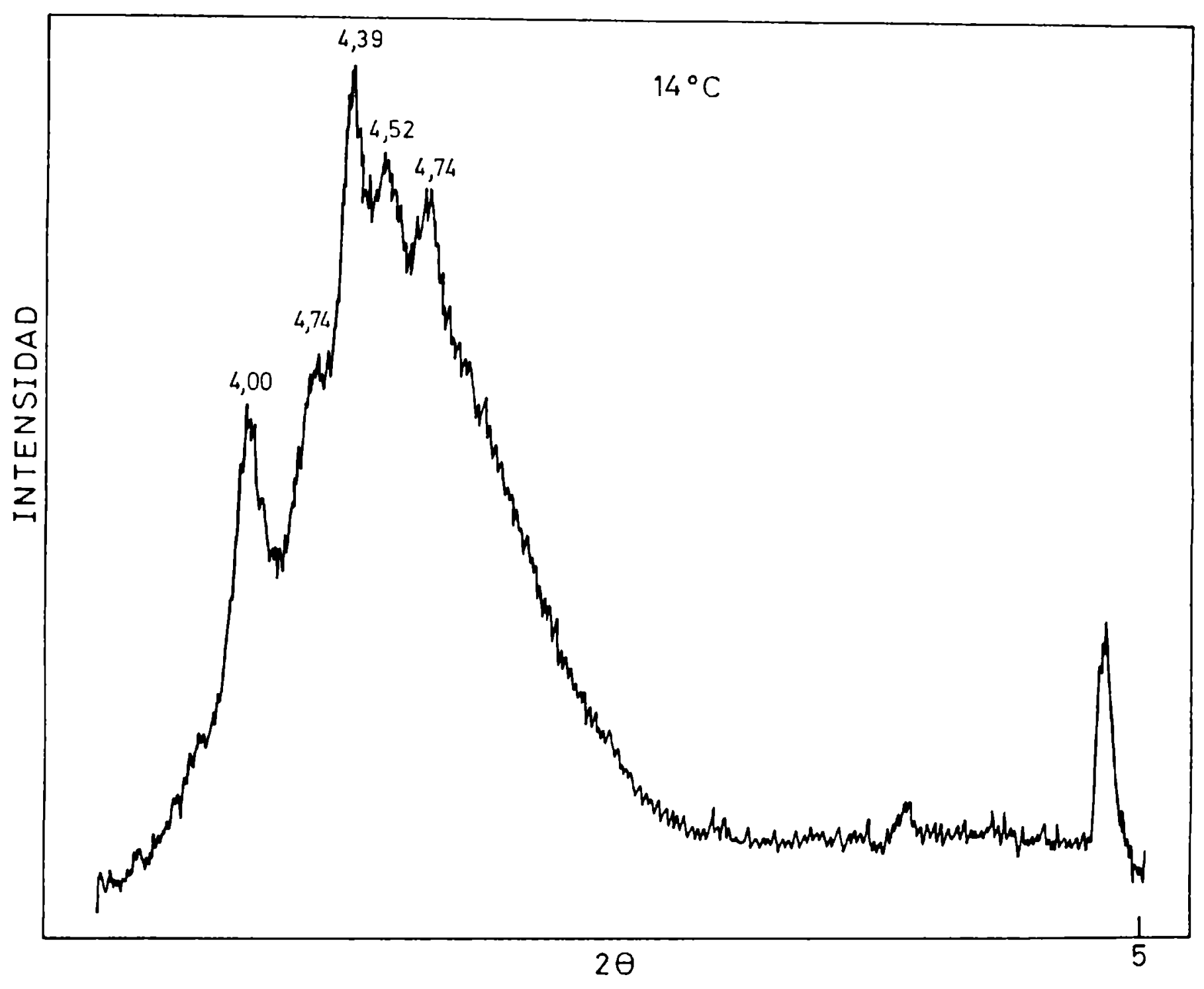

(b)

Figura 22. Espectros de rayos $X$ de las muestras cristalizadas a $38^{\circ} \mathrm{C}, 24^{\circ} \mathrm{C}$ y $14^{\circ} \mathrm{C}$ en forna dinánica (a) y de la muestra $14^{\circ} \mathrm{C}$ cristalizada en forma estática (b) 


\subsubsection{Bspectroscopia Infrarroja.}

Con el objeto de conocer el contenido en isómeros trans de las fracciones sólidas obtenidas en la experiencia de fraccionamiento se realizaron estudios de espectroscopia infrarroja. La Figura 23 presenta tres espectros de infrarrojo pertenecientes a las muestras cristalizadas a $38^{\circ} \mathrm{C}, 24^{\circ} \mathrm{C}$ y $14^{\circ} \mathrm{C}$ (segunda experiencia). Las medidas de las áreas relativas señalan que la probabilidad de separar isómeros trans en los sólidos a las distintas temperaturas es del mismo orden $42,7 \%, 43,2 \%$ y $42,7 \%$ respectivamente. Sin embargo como habiamos explicado el contenido de ácido oleico decrece al descender la temperatura, es decir que la muestra cristalizada a $14^{\circ} \mathrm{C}$ presenta un contenido de isomeros trans menor que la cristalizada a $38^{\circ} \mathrm{C}$. Esto es concordante con el hecho que el diagrama calorimétrico de la fracción sólida a $14^{\circ} \mathrm{C}$ no presenta componentes con puntos de fusión tan altos como los que se encuentran en 1 a fracción $38^{\circ} \mathrm{C}$. La temperatura de pico mayor registrada para la muestra cristalizada a $14^{\circ} \mathrm{C}$ es $36,8^{\circ} \mathrm{C}$ y para la muestra obtenida a $38^{\circ} \mathrm{C}$ es $44,9^{\circ} \mathrm{C}$. Cuando se analizaron las muestras obtenidas en la primer experiencia se encontraron resultados similares.

Si no existiera un contenido tan elevado de ácido elaidico en las muestras sólidas no sería posible justificar los puntos de fusion encontrados por calorimetria diferencial de barrido. De acuerdo a la composición en triglicéridos propuesta por nosotros no deberia haber componentes con puntos de fusión mayores de $30^{\circ} \mathrm{C}$ si todos los ácidos grasos fueran cis. Para ejemplificar el rol que juega una insaturación en la disminución del punto de fusión 
tomemos el ejemplo del triglicérido POP. El mismo funde a temperatura ambiente, $40^{\circ} \mathrm{C}$ antes que PPP; es decir que el reemplazo de un ácido palmitico por un ácido oleico puede modificar en forma notoria la temperatura de fusión. El triglicerido EEE funde a $42,0^{\circ} \mathrm{C} ; \quad \operatorname{SSE}$ y $\mathrm{SEE}$ entre $42,0^{\circ} \mathrm{C}$ y $73,0^{\circ} \mathrm{C}$. Es por esta razón que se encuentran constituyentes con temperaturas superiores a $30^{\circ} \mathrm{C}$ en porcentajes importantes.

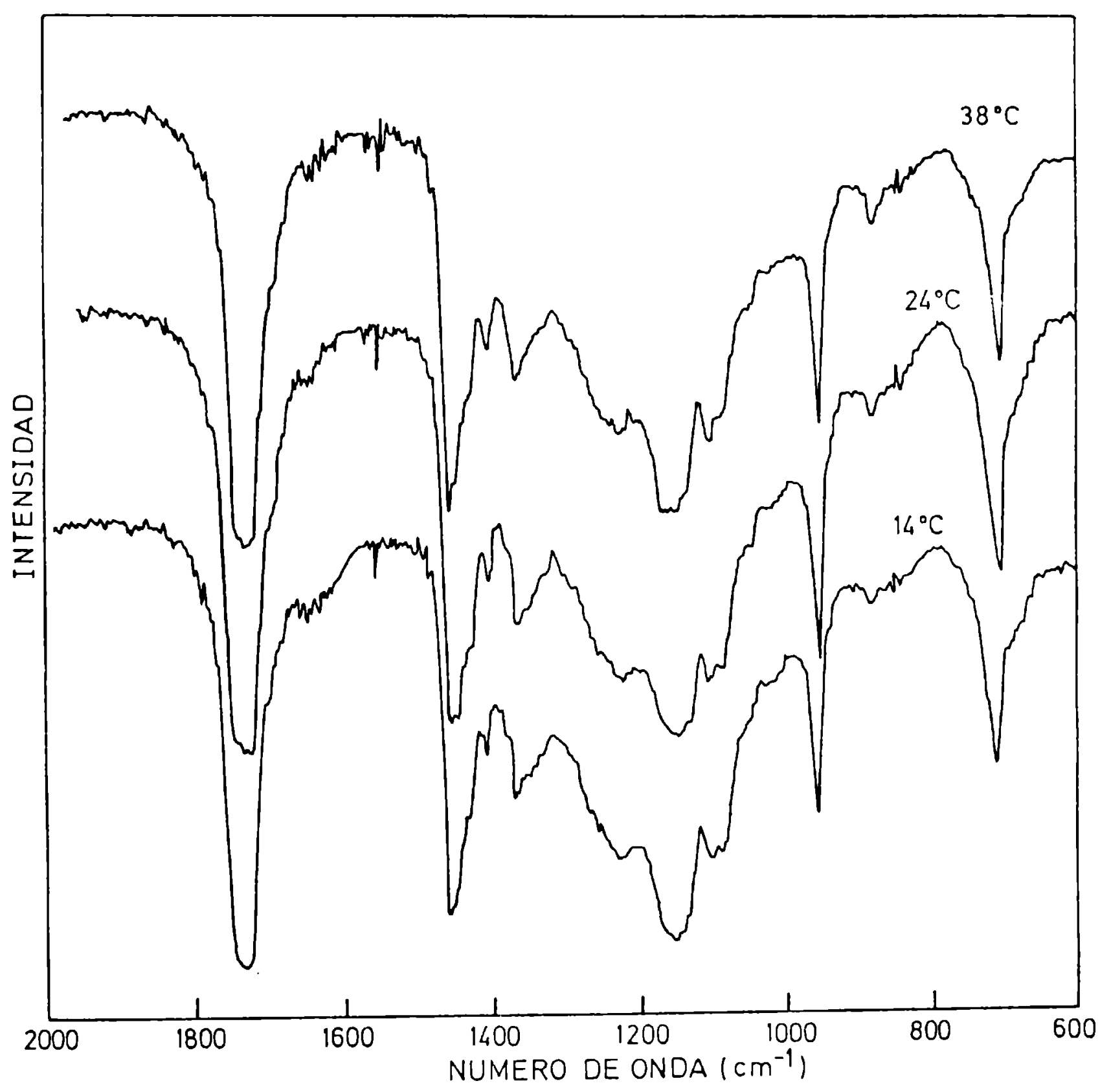

Figura 23. Espectros de infrarrojo de las fracciones sólidas cristalizadas a $38^{\circ} \mathrm{C}, 24^{\circ} \mathrm{C}$ y $14^{\circ} \mathrm{C}$ (Segunda experiencia). 


\subsection{Caracterización de las fracciones liquidas.}

Con el objeto de completar el estudio sobre el fraccionamiento de aceite de girasol hidrogenado se procedió a analizar las fracciones liquidas empleando las mismas técnicas que para caracterizar las muestras originales y las fracciones sólidas. Todos los resultados que se presentan a continuación están referidos a la segunda experiencia.

\subsubsection{Deterninación cuantitativa de ácidos grasos por cromatogra-} fia gaseosa.

En la Figura 24 se ha graficado el porcentaje de cada ácido graso en funcion de la temperatura de cristalización. Las muestras liquidas mostraron hasta la fracción de $22^{\circ} \mathrm{C}$ un incremento del ácido palmitico respecto de la muestra original y por debajo de esta temperatura una pequeña disminución alcanzando en la fracción colectada a $12^{\circ} \mathrm{C}$ valores de $B, 3 \%$. El ácido esteárico para la fracción de $38^{\circ} \mathrm{C}$ se encontró en un valor del orden de la muestra original y luego disminuyó paulatinamente al descender la temperatura de fraccionamiento. Para la fracción de $12^{\circ} \mathrm{C}$ se registró un valor de $8,2 \%$. El ácido oleico se hallo en un porcentaje similar al de la muestra original en la fracción de $38^{\circ} \mathrm{C}$; en el resto de las fracciones descendió al disminuir la temperatura. Para la fracción de $12^{\circ} \mathrm{C}$ el contenido resultó ser $65,2 \%$. El ácido linoleico comenzó con valores semejantes al de la muestra original y se incrementó al descender la temperatura de fraccionamiento 
l legando en la fracción de $12^{\circ} \mathrm{C}$ a un valor de $19,9 \%$.

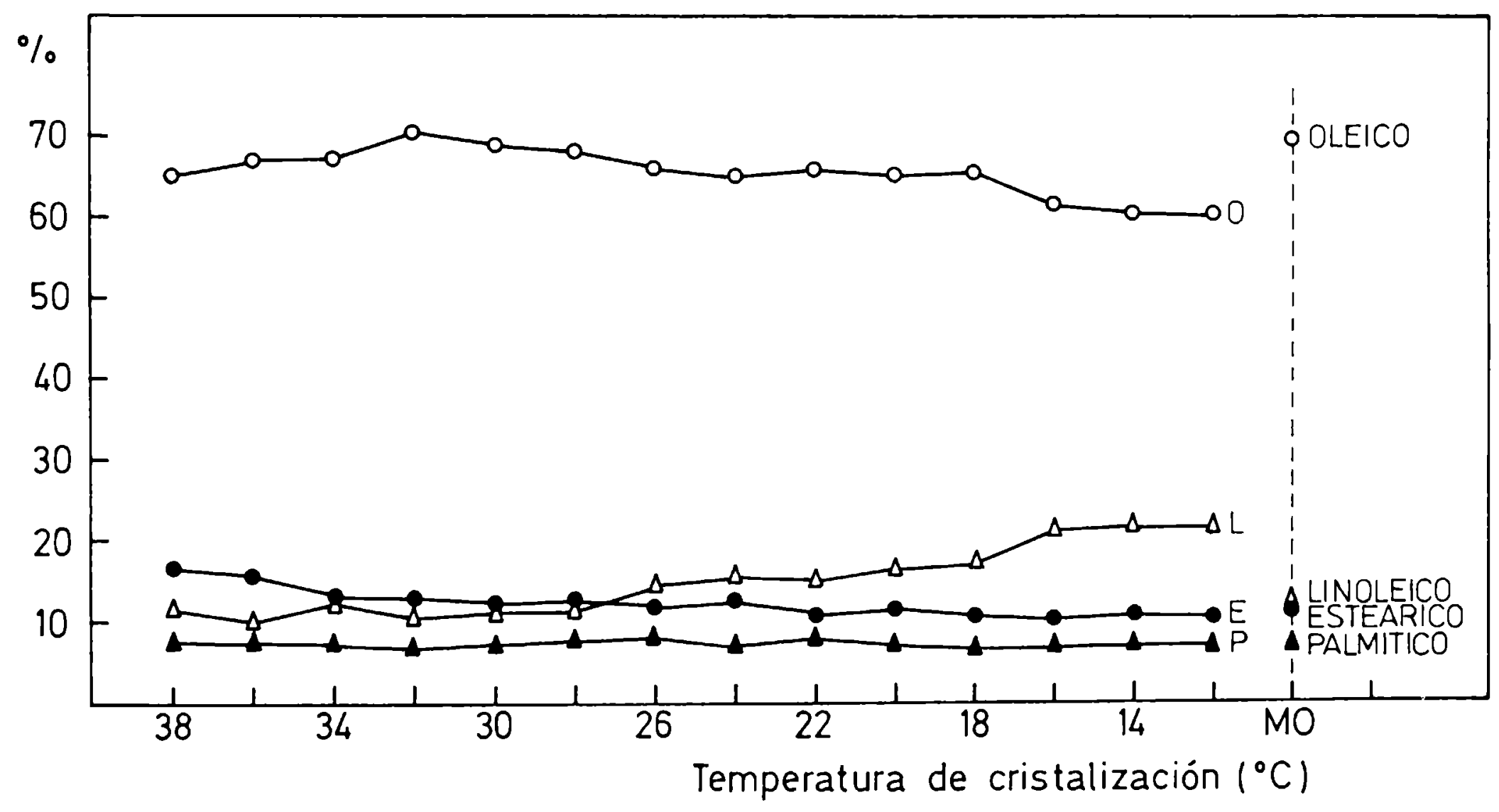

Figura 24. Porcentaje de cada ácido graso en función de la tempcratura de cristalización. 
La comparación entre los contenidos de ácidos grasos entre sólidos y liquidos pone de manifiesto que el ácido palmitico se encontró en cantidades iguales en sólidos y liquidos hasta la fracción de $22^{\circ} \mathrm{C}$ a partir de la cual se ve una pequeña disminución en las fracciones liquidas respecto de los sólidos. El contenido de ácido esteárico para todas las temperaturas fue mayor en las fracciones sólidas que en las liquidas. A modo de ejemplo en la fracción de $38^{\circ} \mathrm{C}$ el ácido esteárico representó el $16,3 \%$ en el sólido y el 11,3\% en el liquido, mientras que en la fracción de $12^{\circ} \mathrm{C}$ se encontró $10,6 \%$ en el sólido y $8,2 \%$ en el liquido. La cantidad de ácido oleico presente en las fracciones liquidas colectadas a $12^{\circ} \mathrm{C}, \quad 14^{\circ} \mathrm{C}$ y $16^{\circ} \mathrm{C}$ es superior al de las fracciones sólidas en aproximadamente $8,4 \%$. Para las otras temperaturas las fracciones liquidas muestran un incremento pequeño respecto de las sólidas. El ácido linoleico se halla incrementado en la fracción liquida con respecto a la sólida correspondiente a la misma temperatura de cristalización, exceptuando las fracciones de $12^{\circ} \mathrm{C}, 14^{\circ} \mathrm{C}$ y $16^{\circ} \mathrm{C}$. Las diferencias que se observaron entre sólidos y liquidos son las esperadas.

\subsubsection{Deterainación cuantitativa de triglicéridos por cronatogra-} fia liquida de alta presión.

Los resultados hallados al analizar los cromatogramas de las muestras liquidas se resumen en la Tabla 9 . En el caso de los liquidos al igual que en los sólidos son pocos los triglicéridos presentes en porcentajes superiores al $10 \%$. 
Tabla 9

Composición porcentual en triglicéridos de las fracciones liquidas correspondientes a la segunda experiencia

\begin{tabular}{|c|c|c|c|c|c|c|c|c|c|}
\hline Temp & & & $\mathrm{Ti}$ & pos d & retenc & (min & & & \\
\hline$\left({ }^{\circ} \mathrm{C}\right)$ & 4,0 & 5,1 & 6,5 & 8,8 & 12,5 & 16,4 & 21,5 & 29,1 & 39,8 \\
\hline 38 & $<1,0$ & $<1,0$ & 1,1 & 2,8 & 18,3 & 39,7 & 29,4 & 6,6 & $<1,0$ \\
\hline 36 & $<1,0$ & $<1,0$ & 1,0 & 2,8 & 18,4 & 38,9 & 29,4 & 6,4 & $<1,0$ \\
\hline 34 & $<1,0$ & 1,2 & 1,3 & 2,8 & 18,4 & 40,2 & 29,4 & 6,1 & $<1,0$ \\
\hline 32 & $<1,0$ & $<1,0$ & 1,5 & 2,8 & 18,5 & 40,3 & 29,2 & 6,0 & $<1,0$ \\
\hline 30 & $<1,0$ & $<1,0$ & 1,1 & 2,8 & 18,6 & 40,4 & 29,3 & 5,9 & $<1,0$ \\
\hline 28 & $<1,0$ & 1,1 & 1,1 & 2,7 & 18,6 & 40,5 & 29,4 & 5,8 & $<1,0$ \\
\hline 26 & $<1,0$ & $<1,0$ & $<1,0$ & 2,6 & 19,2 & 42,0 & 29,6 & 5,9 & $<1,0$ \\
\hline 24 & $<1,0$ & $<1,0$ & 2,0 & 2,1 & 18,6 & 41,0 & 28,1 & 5,7 & $<1,0$ \\
\hline 22 & $<1,0$ & 1,1 & 1,2 & 2,0 & 18,8 & 41,2 & 30,0 & 5,7 & $<1,0$ \\
\hline 20 & $<1,0$ & 1,5 & 1,1 & 1,8 & 18,5 & 41,4 & 30,1 & 5,7 & $<1,0$ \\
\hline 18 & $<1,0$ & 1,1 & 1,4 & 1,7 & 18,3 & 41,4 & 30,2 & 5,7 & $<1,0$ \\
\hline 16 & $<1,0$ & 1,1 & 1,4 & 1,6 & 18,2 & 41,5 & 30,1 & 5,5 & $<1,0$ \\
\hline 14 & $<1,0$ & $<1,0$ & 1,8 & 1,4 & 18,1 & 41,7 & 30,4 & 5,5 & $<1,0$ \\
\hline 12 & $<1,0$ & $<1,0$ & 1,0 & 1,0 & 18,1 & 42,1 & 30,9 & 5,6 & $<1,0$ \\
\hline
\end{tabular}

Al igual que se ha explicado cuando se analizaron los cromatogramas de los sólidos cabe señalar que cada uno de los picos asignado a un triglicérido nayoritario puede contener otros en 
pequeñas cantidades.

SOO: este triglicérido presentó un valor de $6,6 \%$ a $38^{\circ} \mathrm{C}$ y 1 uego descendió en un $1 \%$ a 10 largo de las fracciones registrándose en un $5,6 \%$ a $12^{\circ} \mathrm{C}$.

POO: este triglicérido se encontró a $38^{\circ} \mathrm{C}$ en porcentajes de $29,4 \%$ y luego aumentó al disminuir la temperatura de cristalización hasta 1 legar a $30,9 \%$ a $12^{\circ} \mathrm{C}$.

000: su contenido a $38^{\circ} \mathrm{C}$ fue de $39,7 \%$ aumentando al disminuir la temperatura de fraccionamiento alcanzando a $12^{\circ} \mathrm{C}$ valores de $42,1 \%$.

LOO: a $38^{\circ} \mathrm{C}$ se presentó en un 18,3\%; al descender la temperatura de cristalización $y$ hasta $26^{\circ} \mathrm{C}$ fue aumentando y alcanzó un valor de $19,2 \%$. Por debajo de la mencionada temperatura disminuyó hasta $18,1 \%$ \& $12^{\circ} \mathrm{C}$

LLP: comenzando con el contenido hallado para la muestra original (2,8\%) disminuyó al descender la temperatura de fraccionamiento en un $1,8 \%$ presentando a $12^{\circ} \mathrm{C}$ un valor de $1,0 \%$.

LLO: permanece constante en un porcentaje pequeño, alrededor del $1 \%$.

En la Tabla 9 se ve claramente que los cambios en la composición quimica de los liquidos son extremadamente pequeños. Si tenemos en cuenta que al finalizar el fraccionamiento solo se han 
retirado $7 \mathrm{Kg}$ de los $27 \mathrm{Kg}$ que se cargaron al tangue del equipo fraccionador $y$ que los cambios en los solidos si bien son más notorios son graduales es esperable este resultado.

El ácido graso esteárico probablemente forme parte del triglicérido SOL de igual tiempo de retención que POO por lo que eluirtan como un único pico. Asi en sólidos y liquidos podria modificarse las proporciones en gue estos triglicéridos están presentes. En nuestros cromatogramas no fueron detectados triglicéridos formados por ácido esteárico en porcentajes significativos. Esto coincide con el hecho que se registraron porcentajes menores de ácido estérico en los liquidos.

Comparando los resultados hallados para solidos y liguidos se puede destacar que en los liquidos partiendo de la composición original los contenidos de triglicéridos se modificaron ligeramente mientras que en los sólidos en las fracciones superiores y hasta $30^{\circ} \mathrm{C}$ se registró un enriquecimiento en los triglicéridos de ECN mayor con respecto al resto de los triglicéridos componentes de las fracciones. Por debajo de dicha temperatura en cambio se incrementaron los triglicéridos con menores ECN. Por otra parte el comportamiento presentado por los distintos triglicéridos a lo largo del fraccionamiento resultó diferente. Mientras que en los sólidos poo disminuyó al descender la temperatura de cristalización en los liguidos presentó un ligero aumento. Asi mismo en las fracciones sólidas 000 aumentó hasta $30^{\circ} \mathrm{C}$ y por debajo de dicha temperatura descendió en tanto que en los liguidos se encontró un paulatino aumento al descender la temperatura. LLP aumentó en los sólidos y disminuyó en los liquidos mientras gue LOO aumentó en los sólidos a todas las temperaturas y en los liguidos hasta 
$26^{\circ} \mathrm{C}$; por debajo de esta temperatura los liquidos registraron un descenso del 1\%. SOO en cambio presento la mlsma tendencia en sólidos y liquidos, disminuyo al descender la temperatura de cristalización.

\subsubsection{Resonancia magnética nuclear}

Las fracciones liquidas al igual que las sólidas fueron caracterizadas mediante esta técnica. En la Tabla 10 se resumen los resultados arrojados al estudiar las fracciones de $38^{\circ} \mathrm{C}, 24^{\circ} \mathrm{C}$ y $14^{\circ} \mathrm{C}$ pertenecientes a cada uno de los grupos señalados.

Tabla 10

Resonancia magnética nuclear de las fracciones $38^{\circ} \mathrm{C}, 24^{\circ} \mathrm{C}$ y $14^{\circ} \mathrm{C}$

\begin{tabular}{|ccccc|}
\hline Muestrg. & $\begin{array}{c}\text { numero de } \\
\text { protones } \\
\text { olefinicos }\end{array}$ & $\begin{array}{c}\text { numero de } \\
\text { protones } \\
\text { totgles }\end{array}$ & medio & de iodo \\
$38^{\circ} \mathrm{C}$ & 4 & 86 & 746 & 68 \\
$24^{\circ} \mathrm{C}$ & 4 & 80 & 708 & 84 \\
$14^{\circ} \mathrm{C}$ & 6 & 78 & 700 & 101 \\
\hline
\end{tabular}


Las muestras liquidas presentaron un peso molecular medio inferior que los sólidos cristalizados a la misma temperatura en todas las fracciones. Asi mismo el indice de iodo también resultó superior es decir que los liquidos poseen un contenido mayor de ácidos grasos insaturados. Si comparamos los liquidos entre si podemos destacar que a $14^{\circ} \mathrm{C}$ el contenido de ácidos grasos insaturados debe ser superior que a $38^{\circ} \mathrm{C}$. Esto es concordante con el hecho que el ácido linoleico se incrementa notoriamente al descender la temperatura. Como era de esperar el número de protones totales disminuye al aumentar el número de protones olefinicos.

\subsubsection{Calorinetria diferencial de barrido.}

La Figura 25 presenta tres diagramas correspondientes a la muestra original, la fracción sólida a $38^{\circ} \mathrm{C}$ y la fracción liquida colectada a la misma temperatura correspondientes a la segunda experiencia de fraccionamiento. Tanto el diagrama del sólido como el del liquido son diferentes al de la muestra original. Dado que a $38^{\circ} \mathrm{C}$ se extrajeron del filtro $333,0 \mathrm{~g}$ y en el tanque se cargaron $27 \mathrm{Kg}$ de aceite hidrogenado era de esperar que el perfil de la muestra 1 iquida colectada a $38^{\circ} \mathrm{C}$ fuera similar al de la muestra original. Sin embargo la extracción selectiva de triglicéridos de mayor punto de fusión de la mezcla produjo pequeños cambios en la curva de fusión. De ahI la diferencia que se observa entre ambos perfiles. 


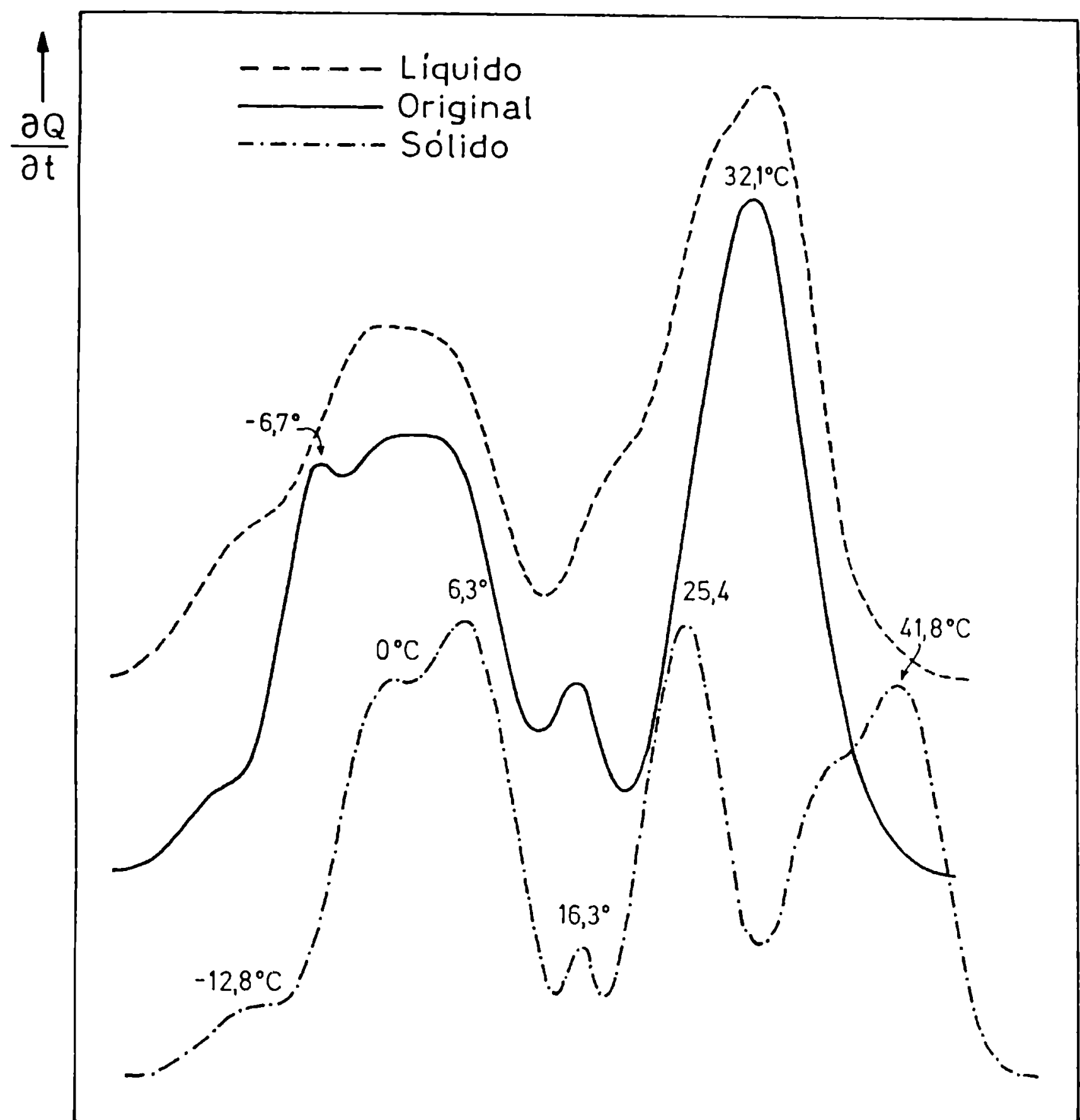

tiempo (min)

Figura 25. Ternogranas de la muestra original y las fracciones liquidas y sólidas a $38^{\circ} \mathrm{C}$ correspondientes a la segunda experiencia de fraccionamiento 
No se obtuvieron ni para las fracciones gólidas ni para las liquidas diagramas de fusión aguda que presentaran rangos de $2^{\circ} \mathrm{C}$ de ancho, es decir que la fusión se produjera en los intervalos de temperaturas seleccionados en resultados y discusión sección 2. El fraccionamiento de girasol hidrogenado no arrojó los mismos resultados que se obtienen cuando se fracciona aceite de palma en el siguiente sentido: si la temperatura de cristalización seleccionada resultó por ejemplo ser $20^{\circ} \mathrm{C}$, serla esperable que la fracción sólida presente constituyentes que fundan por encima de esa temperatura y la fracción Iiquida componentes que fundan por debajo. Sin embargo, las muestras sólidas mostraron perfiles similares a la muestra original en las temperaturas por debajo de la de cristalización y se vieron enriquecidas en sólidos por encima de dicha temperatura mientras que las muestras liquidas presentaron porcentajes bajos de triglicéridos de puntos de fusión superiores a la temperatura de fraccionamiento. El hecho que los sólidos contengan triglicéridos de menor punto de fusión que la temperatura de cristalización puede deberse a que dada la similitud de tamaño y naturaleza electroquimica de las moléculas de triglicéridos, triglicéridos de menor punto de fusión ocupen sitios en la red sustituyendo a otros de mayor punto de fusión. Además la fase sólida puede retener liquido el cual puede ser resfonsable de los picos de menor temperatura de fusión observados.

La Tabla 11 muestra los porcentajes de liquido correspondientes a la muestra original y a las fracciones sólidas y líquidas obtenidas a $14^{\circ} \mathrm{C}, 24^{\circ} \mathrm{C}$ y $38^{\circ} \mathrm{C}$ a diferentes temperaturas. Esta tabla fue construida integrando el área bajo la curva en los termo- 
gramas hasta las temperaturas que alli figuran suponiendo que el calor intercambiado representa solo la fusion de diferentes constituyentes. El contenido de sólidos a una determinada temperatura es mayor siempre en la fracciones solidas que en las liquidas correspondientes y comparando las fracciones solidas entre sí se encuentra que especialmente las de mayor temperatura estan enriquecidas en solidos respecto de las cristalizadas a temperaturas menores. Las fracciones pertenecientes al primer grupo poseen un contenido de sólidos mayor que la muestra original.

Tabla 11

Porcentaje de aceite liquido en sólidos, liquidos y nuestra original

\begin{tabular}{|c|c|c|c|c|c|c|c|}
\hline \multirow{2}{*}{$\begin{array}{l}\text { Temp } \\
\left({ }^{\circ} \mathrm{C}\right)\end{array}$} & \multicolumn{7}{|c|}{ Porcentaje de aceite } \\
\hline & $\mathrm{MO2}$ & S38 & L 38 & $\mathrm{~S} 24$ & L 24 & S14 & L 14 \\
\hline 0,0 & 20,8 & 13,3 & 20,4 & 24,9 & 29,0 & 28,2 & 34,0 \\
\hline 5,0 & 37,9 & 31,3 & 36,3 & 35,2 & 31,6 & 43,2 & 45,4 \\
\hline 10,0 & 41,8 & 35,0 & 39,7 & 39,7 & 44,3 & 48,5 & 62,6 \\
\hline 15,0 & 52,5 & 45,0 & 50,6 & 43,5 & 59,5 & 59,0 & 78,4 \\
\hline 20,0 & 53,3 & 55,4 & 54,7 & 53,7 & 74,1 & 59,9 & 82,4 \\
\hline 25,0 & 55,0 & 57,6 & 59,9 & 55,0 & 79,7 & 66,5 & 86,3 \\
\hline 30,0 & 73,0 & 59,8 & 75,8 & 62,1 & 82,5 & 72,3 & 94,6 \\
\hline 35,0 & 82,0 & 60,2 & 86,0 & 72,3 & 84,1 & 89,0 & 89,8 \\
\hline 40,0 & 84,2 & 61,0 & 88,6 & 84,4 & 98,8 & --- & - \\
\hline
\end{tabular}


En la Figura 26 se presentan los termogramas interpretados ror computadora pertenecientes a los liquidos colectados a $14^{\circ} \mathrm{C}$, $24^{\circ} \mathrm{C}$ y $38^{\circ} \mathrm{C}$. La fracción $38^{\circ} \mathrm{C}$ se interpretó con seis constituyentes de temperaturas de pico $-11,9^{\circ} \mathrm{C} ;-1,7^{\circ} \mathrm{C} ; 6,7^{\circ} \mathrm{C} ; 20,9^{\circ} \mathrm{C} ; 28,3^{\circ} \mathrm{C}$ y $34,8^{\circ} \mathrm{C}$ con áreas porciento iguales a 9,$0 ; 18,5 ; 17,1 ; 10,6 ; 18,0$ y 26,8. La fracción $24^{\circ} \mathrm{C}$ se ajustó sumando seis constituyentes de temperaturas de pico $-13,8^{\circ} \mathrm{C} ; \quad-5,1^{\circ} \mathrm{C} ; \quad 5,5^{\circ} \mathrm{C} ; \quad 14,1^{\circ} \mathrm{C} ; \quad 28,3^{\circ} \mathrm{C}$ y $34,9^{\circ} \mathrm{C}$ y áreas porciento 7,$5 ; 11,5 ; 26,3 ; 31,8 ; 11,1$ y 11,8 . La fracción $14^{\circ} \mathrm{C}$ resultó la suma de seis componentes de temperatura de pico $-16,9^{\circ} \mathrm{C} ; \quad-7,8^{\circ} \mathrm{C} ; \quad 0,9^{\circ} \mathrm{C} ; \quad 8,8^{\circ} \mathrm{C} ; \quad 23,6^{\circ} \mathrm{C} ; \quad 29,8^{\circ} \mathrm{C}$ y áreas porciento 7,$9 ; 14,6 ; 22,9 ; 34,1 ; 9,8$ y 10,7 . Las fracciones sólidas dieron diagramas más anchos que los liquidos a la misma temperatura y a medida que la temperatura de cristalización fue disminuyendo los diagramas de los sólidos se fueron angostando hasta llegar a ser menores que en la muestra original. 


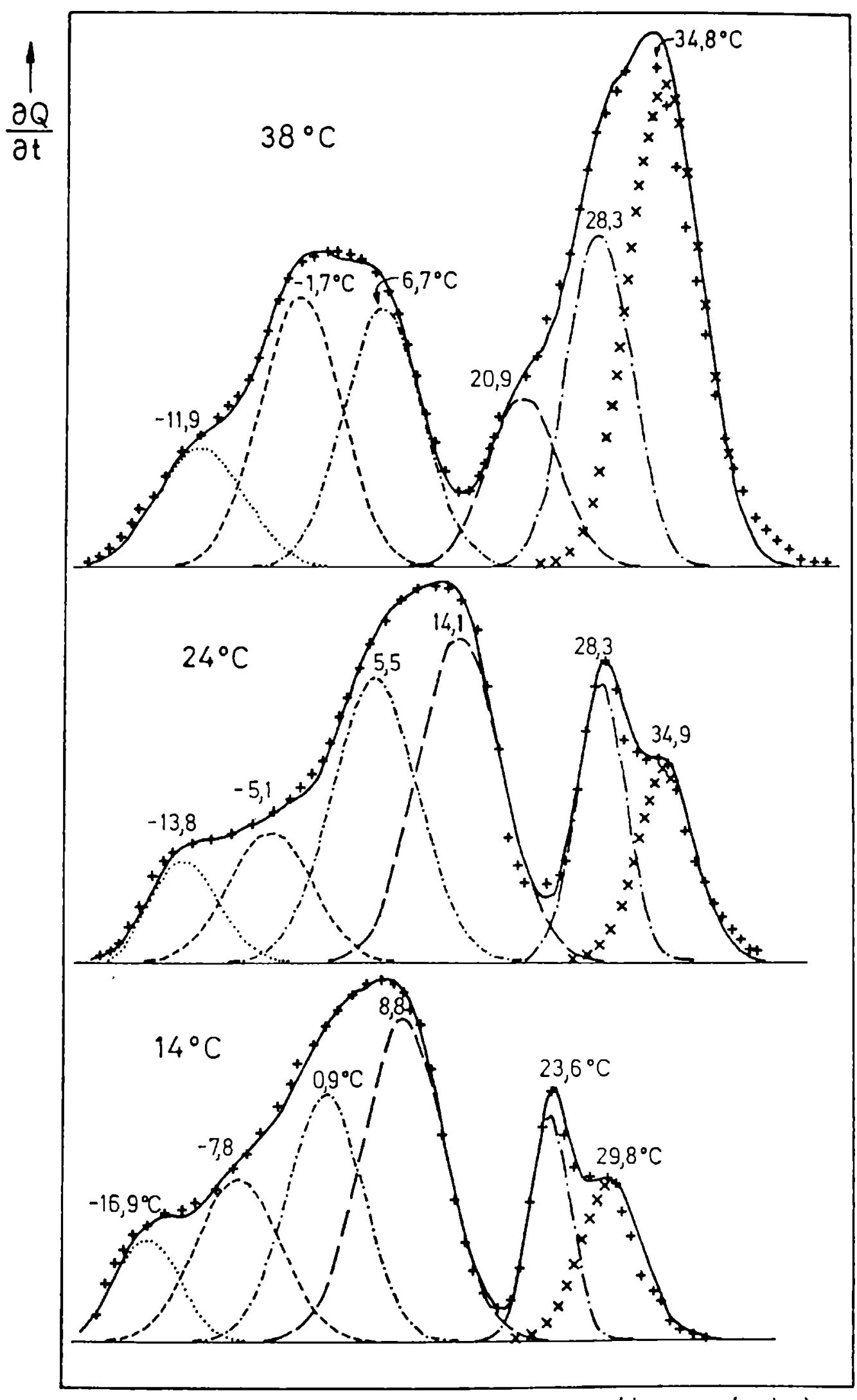

tiempo $(\min )$

Figura 26. Ternogranas correspondientes a las fracciones liquidas colectadas a $38^{\circ} \mathrm{C}, 24^{\circ} \mathrm{C}$ y $14^{\circ} \mathrm{C}$ interpretados por conputadora. 
Las entalpias de cada constituyente y las entalpias totales de las muestras se calcularon como se explicó previamente (Materiales y Metodos sección 5.5). Las entalpias totales para las fracciones $38^{\circ} \mathrm{C}, \quad 24^{\circ} \mathrm{C}$ y $14^{\circ} \mathrm{C}$ resultaron ser 78,$5 ; 63,0$ y 54,8 Joule/g.

La comparación entre estos valores y los hallados para los sólidos permite destacar que las muestras sólidas tienen entalpias totales mayores que las liquidas a la misma temperatura. A medida que la temperatura fue menor las entalpias totales descendieron. Esto habla de triglicéridos más saturados y formas cristalinas de mayor estabilidad en los sólidos y en las fracciones de temperaturas superiores.

\subsubsection{Espectroscopia infrarroja}

Resulta llamativo el hecho que una variación pequeña en las concentraciones de los triglicéridos constituyentes determine una variación tan marcada en la consistencia. Las fracciones sólidas tenian la consistencia de sólidos amorfos y los liquidos una apariencia similar al aceite. En el proceso de hidrogenación se ve favorecida la formación de isómeros trans. Por lo tanto en el aceite hidrogenado resultante tendremos una mezcla de isómeros geométricos de los ácidos oleico y linoleico que se distribuirán de diferente manera en los sólidos que en los líquidos. Si tenemos presente que los puntos de fusión de los isómeros del ácido oleico cis $\left(13,4^{\circ} \mathrm{C} a ; 16,3^{\circ} \mathrm{C} \beta\right)$ y del trans $\left(44,5^{\circ} \mathrm{C}\right)$ difieren en $20^{\circ} \mathrm{C} y$ los correspondientes al ácido linoleico (isómero cis $-5,2^{\circ} \mathrm{C}$ a 
$-5,0^{\circ} \mathrm{C}$ y trans $28^{\circ} \mathrm{C}$ a $29^{\circ} \mathrm{C}$ ) en aproximadamente $33^{\circ} \mathrm{C}$, de acuerdo a la distribución de estos isomeros esperamos diferentes puntos de fusion para las fracciones. Los liquidos presentaron espectros infrarrojos diferentes a los sólidos: un pico de absorción de los isómeros cis ancho y un pico agudo de isómeros trans. Este pico se encontró en un $24,1 \%$ a $38^{\circ} \mathrm{C}$ y luego descendió al descender la temperatura a valores de $21,9 \%$ y $19,7 \%$ a $24^{\circ} \mathrm{C}$ y $14^{\circ} \mathrm{C}$ (Figura 27 ). La disminución en el contenido de isóneros trans es en realidad mayor que la que surge de estos valores ya que debemos considerar que al descender la temperatura de fraccionamiento el contenido de ácido oleico decrece de fracción en fracción.

Si comparamos los valores encontrados para liquidos y sólidos es claro el menor contenido de isómeros trans presentes en los liquidos. Es decir que en los sólidos fuimos retirando selectivamente los isómeros de mayor punto de fusión enriqueciéndose los liquidos remanentes en isómeros cis. Esto es concordante con lo observado cuando se analizaron estas muestras por calorimetria diferencial de barrido. Las muestras liquidas según se explicó presentaron diagramas de fusión más angostos y pocos constituyentes por encima de la temperatura de fraccionamiento, es decir presentaron componentes con menores puntos de fusión. 


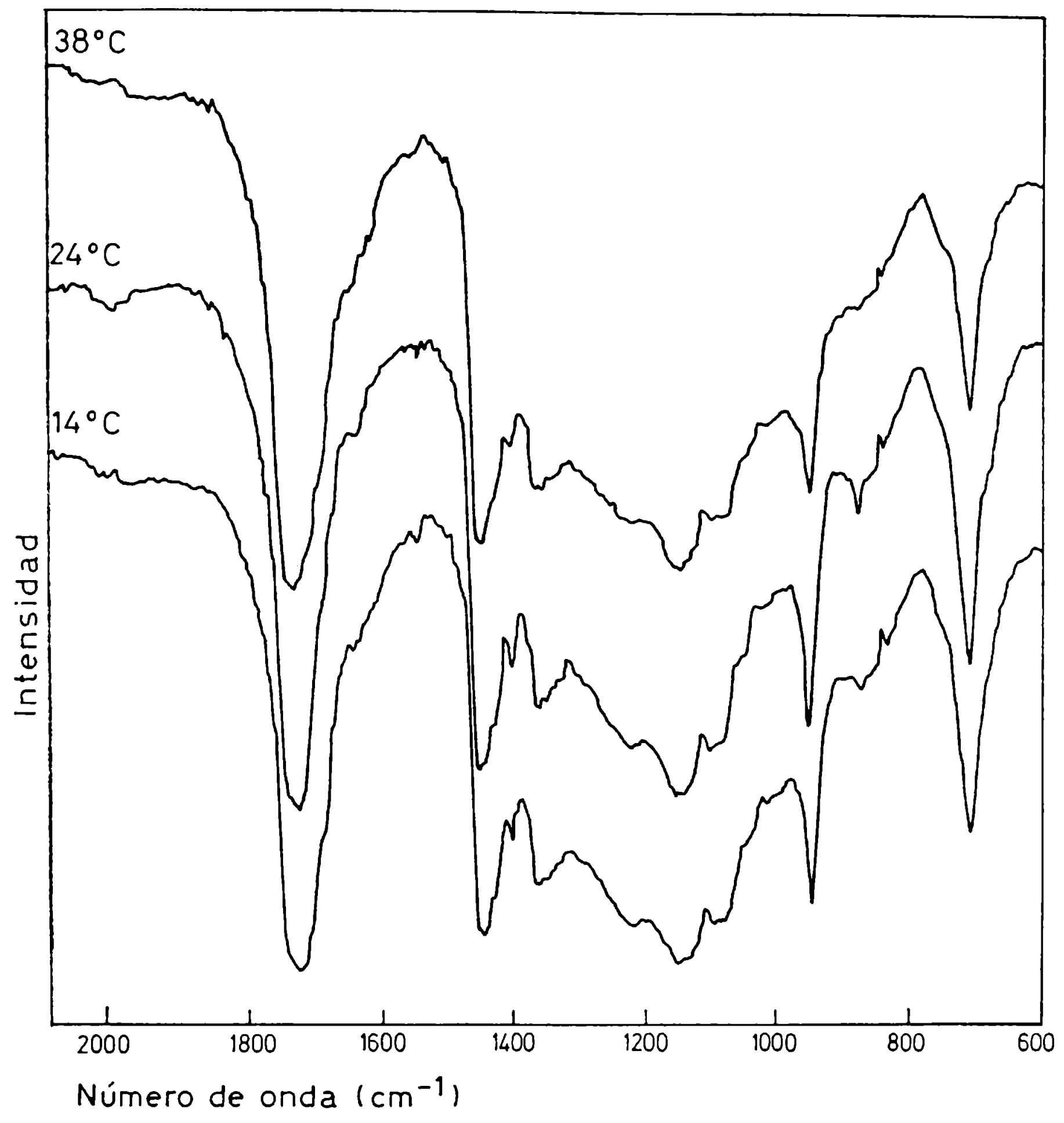

Figura 27. Espectros de infrarrojo de las muestras liquidas colectadas a $38^{\circ} \mathrm{C}, 24^{\circ} \mathrm{C}$ y $14^{\circ} \mathrm{C}$ (Segunda experiencia) 
El sistema que estamos analizando posee por lo menos cinco componentes cuya concentración es superior al 3\%. Las condiciones elegidas de trabajo nos permiten tratar al sistema como si estuviera en equilibrio termodinamico ya que se realizo una cristalización lenta dando tiempo suficiente a los triglicéridos para difundir entre el sólido y el liquido. En este sistena de dos fases debe cumplirse para cada temperatura que:

$m_{\mathbf{g}}+m_{1}=M_{T}$

donde

ma es la masa de la fase sólida

mi es la masa de la fase liquida

MT es la masa total cargada en el tanque

y para cada componente:

$$
\sum_{T=T_{i}}^{T_{C}} m_{g T} X_{a T}+m_{1} X_{I}=M_{T} X_{0}
$$

donde

$X_{a} T$ es el porcentaje del componente en la fase sólida en un rango de $2^{\circ} \mathrm{C}$

mat es la masa extraida en dicho intervalo

$X_{1}$ es el porcentaje del componente en la fase liguida a la temperatura de cristalización

$X_{0}$ es el porcentaje del componente en la muestra original. 


$$
\sum_{s=1}^{n} x_{y}=1 \quad(15) \quad y \quad \sum_{l=1}^{n} x_{1}=1 \quad(16)
$$

definimos el coeficiente de partición Kp como

$$
\sum_{P}=\ldots \ldots
$$

m $1 \mathrm{X}_{1}$

El numerador de la ecuación (17) resulta igual a la masa total de sólido a la temperatura de cristalización seleccionada por la concentración que tendria dicho sólido si se hubiera fraccionado el total de la masa cargada al tanque a cada una de las temperaturas elegidas.

De la ecuación (14) podemos despejar esta concentración:

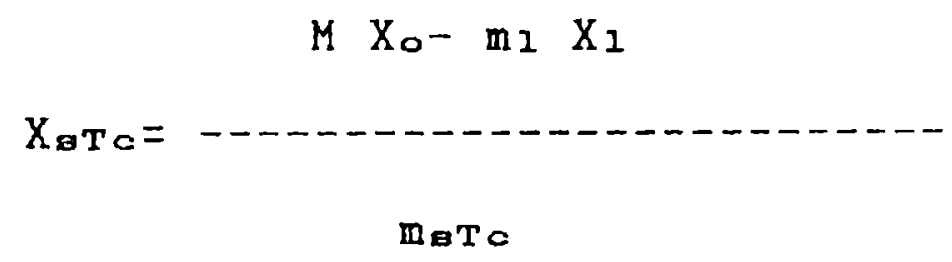

mato

y de la ecuación (17) obtenemos la relación

$$
\text { Xate mi } X_{1}
$$

$\mathrm{K}_{\mathrm{P}}$




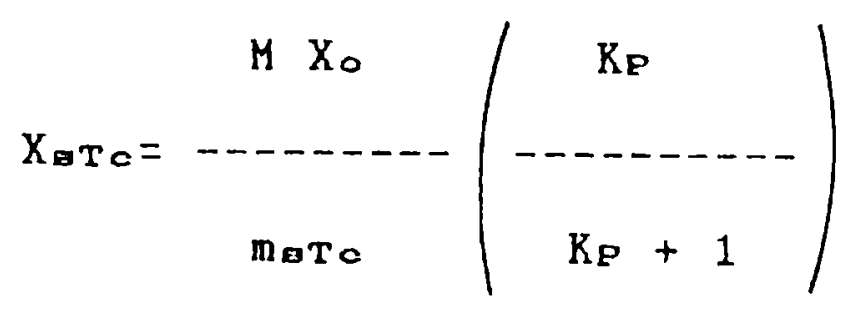

De la misma manera podemos encontrar para los líguidos una expresión similar:

$$
X_{1}=\ldots\left(\begin{array}{c}
M X_{0} \\
m_{1}
\end{array}\right)
$$

Con los valores de $K_{P}$ y conociendo la masa total cargada al tanque y la composición de la muestra original se puede encontrar para cada temperatura un contenido de sólidos tal que los valores de concentración calculados cumplan con (15). Por ejemplo si caleulamos las masas de SOO, POO, 000, LOO y LLP a $31^{\circ} \mathrm{C}$ con estas ecuaciones encontramos valores de $3765,5 \mathrm{~g} ; 966,3 \mathrm{~g} ; 581,1 \mathrm{~g} ; 109,5 \mathrm{~g}$ y 66,3g respectivamente. De los datos experimentales para esta misma temperatura los valores hallados son $3896,4 \mathrm{~g} ; 952,6 \mathrm{~g}$; $593,7 \mathrm{~g} ; 257,1 \mathrm{~g}$ y $140,1 \mathrm{~g}$. Los primeros tres triglicéridos se encuentran en cantidades superiores al $10 \%$ en las muestras mientras que los dos últimos en porcentajes menores que el $5 \%$. El modelo predice con una muy buena aproximación los contenidos de triglicéridos mayoritarios. 
La Tabla 12 resume los $\mathrm{K}_{p}$ para los triglicéridos LLP, LOO, 000, POO y SOO. El triglicérido SSO sólo se encontró en porcentajes mayores al $3 \%$ en $10 s$ sólidos. Los triglicéridos de tiempos de retención inferiores a LLP no fueron importantes ni en los sólidos ni en los liquidos. El estudio se realizó considerando igual respuesta al detector de los cinco triglicéridos y que cada pico se encuentra formado por un sólo triglicérido. En este tipo de sistema la técnica de cromatografia liquida de alta presión empleada por nosotros no es capaz de separar isómeros geométricos, importante a tener en cuenta dada la historia previa de las muestras.

Los valores de $K_{p}$ son semicuantitativos ya que los porcentajes de las áreas se calcularon como ya se explicó trazando perpendiculares a la linea de base dividiendo picos solapados (Frede y Thiele, 1988). En la Figura 28 se representa la relación de partición en función de la temperatura de fraccionamiento. Puede verse que estas relaciones aumentan al disminuir la temperatura de cristalización con diferentes pendientes, es decir el solido se enriguece en soo respecto del liquido en mucho mayor grado que en LOO, 000 y $P 00$ y el contenido de LLP crece en el sólido cristalizado a $12^{\circ} \mathrm{C}$ respecto del sólido a $38^{\circ} \mathrm{C}$ en forma notable. 
Tabla 12

Relaciones de partición para los triglicéridos LLP, L00, 000, POO y 500

\begin{tabular}{|cccccc|}
\hline Terp & \multicolumn{5}{c}{ Tiempos de retencion $(\mathrm{min})$} \\
$\left({ }^{\circ} \mathrm{C}\right)$ & 8,8 & 12,5 & 16,4 & 21,5 & 29.1 \\
\hline 38 & 0,012 & 0,001 & 0,006 & 0,011 & 0,067 \\
36 & 0,026 & 0,011 & 0,016 & 0,028 & 0,124 \\
34 & 0,045 & 0,029 & 0,029 & 0,049 & 0,189 \\
32 & 0,061 & 0,048 & 0,048 & 0,071 & 0,250 \\
30 & 0,082 & 0,062 & 0,067 & 0,095 & 0,299 \\
28 & 0,170 & 0,102 & 0,102 & 0,133 & 0,365 \\
26 & 0,312 & 0,143 & 0,139 & 0,170 & 0,426 \\
24 & 0,624 & 0,203 & 0,190 & 0,215 & 0,519 \\
22 & 0,769 & 0,221 & 0,202 & 0,228 & 0,538 \\
20 & 0,945 & 0,246 & 0,218 & 0,243 & 0,569 \\
18 & 1,051 & 0,258 & 0,228 & 0,252 & 0,582 \\
16 & 1,259 & 0,278 & 0,238 & 0,262 & 0,638 \\
14 & 1,561 & 0,300 & 0,248 & 0,270 & 0,656 \\
12 & 2,876 & 0,332 & 0,264 & 0,282 & 0,671 \\
\hline
\end{tabular}




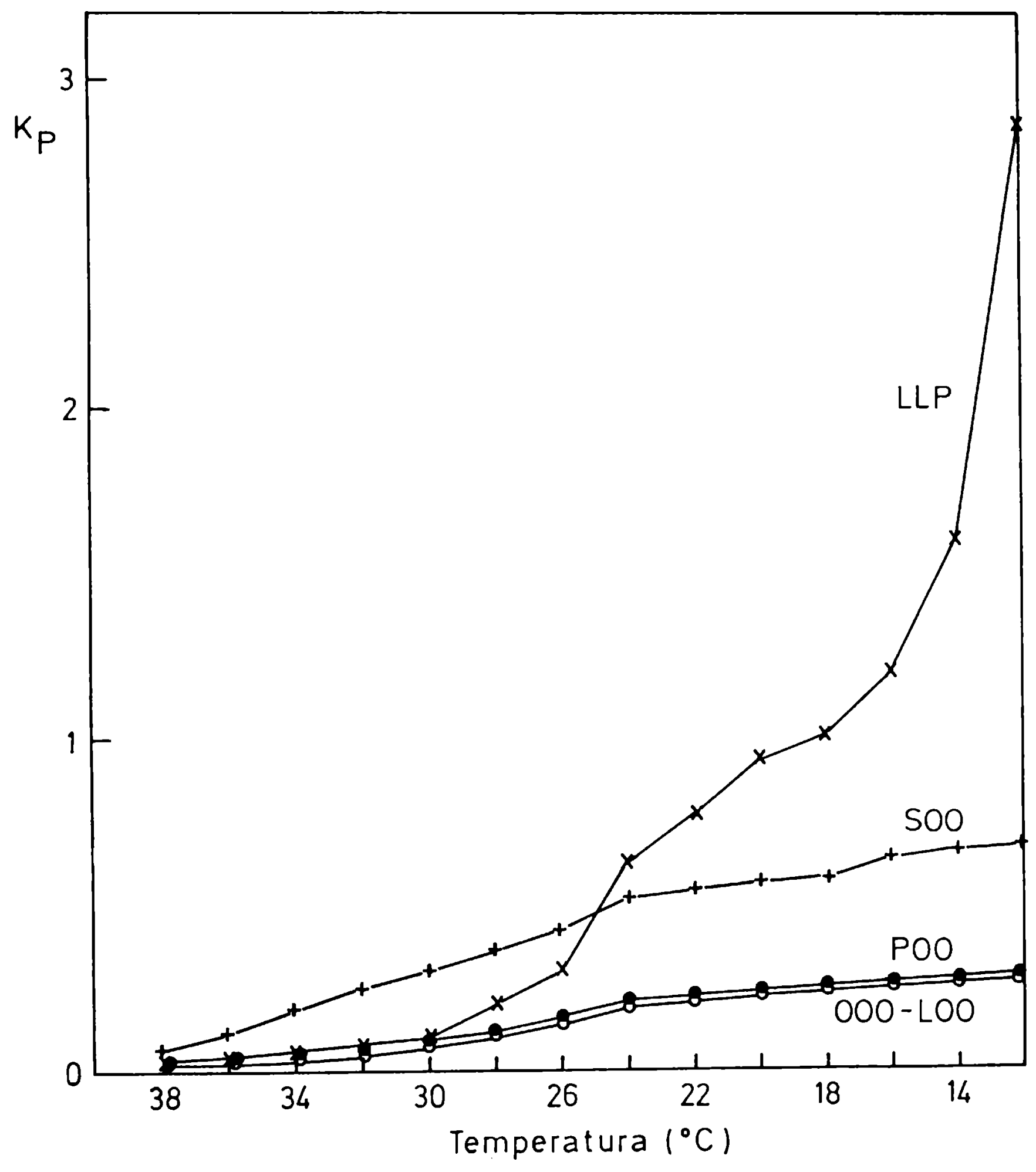

Figura 28. Relaciones de partición en función de la temperatura de cristalización correspondientes a los triglicéridos S00, P00, 000 , LOO y LLP 


\section{INFLUEHCIA DE LA VELOCIDAD DE EHERTAHIENTO}

El aceite de girasol hidrogenado como se explico anteriormente está constituido por una mezcla de triglicéridos que no cristalizan como un compuesto simple. En ambas nuestras originales se presentaron más de un componente con diferentes puntos de fusión. Cuando se fundió SSS de $99,95 \%$ de pureza (Sigma) se encontró un único pico a $71,3^{\circ} \mathrm{C}$. Si este compuesto se enfriaba a una velocidad de $10^{\circ} \mathrm{C} / \mathrm{min}$ aparecian dos picos a $59,5^{\circ} \mathrm{C}$ y $71,5^{\circ} \mathrm{C}$. El pico encontrado inicialmente corresponde a la forma $\beta$; los picos hallados posteriormente a las formas $\beta^{\prime}$ y $\beta$. Bajo estas condiciones el polimorfismo puede ser puesto de manifiesto. Por otra parte si se funden mezclas de SSS y AAA la solubilidad relativa cambia con las proporciones elegidas pudiendo encontrarse uno o dos picos. Por esta razón en todas las fracciones el comportamiento térmico resultó ser muy complicado dadas las distintas interacciones que se producen en los triglicéridos al cambiar su solubilidad con la composición quimica. Todo esto sugiere que la estabilidad de los cristales no depende sólo de la disposición de los triglicéridos en el cristal (forma cristalina) sino también de la cantidad y calidad de los mismos.

Con el objeto de estudiar la influencia de la velocidad de enfriamiento las distintas fracciones fueron fundidas a $80^{\circ} \mathrm{C} y$ posteriormente enfriadas con velocidad lineal o con velocidades exponenciales. La muestra enfriada con velocidad lineal fue la fracción de $37^{\circ} \mathrm{C}$ correspondiente a la primer experiencia de fraccionamiento. $15 \mathrm{mg}$ de esta muestra se colocaron en una cápsula de aluminio sellada y se programó el calorimetro diferencial do 
barrido con velocidades de $0,5^{\circ} \mathrm{C} / \mathrm{min}$ y $20^{\circ} \mathrm{C} / \mathrm{min}$. Las muestras enfriadas con velocidades exponenciales se colocaron en un criostato en el cual circulaba etilenglicol a $-30^{\circ} \mathrm{C}$. Los tubos correspondientes a la velocidad rápida se sumergieron desnudos en el fluido y se registraron los cambios de temperatura con una termocupla. Los tubos correspondientes a velocidades lentas se recubrieron con dos gruesas coberturas de goma y se sumergieron en el fluido registrándose en igual forma las temperaturas. Las muestras asi tratadas fueron las colectadas a $41^{\circ} \mathrm{C}, 35^{\circ} \mathrm{C}, 23^{\circ} \mathrm{C}$ y $13^{\circ} \mathrm{C}$ correspondientes a la primer experiencia y las extraidas del filtro a $36^{\circ} \mathrm{C}, \quad 26^{\circ} \mathrm{C}$ y $16^{\circ} \mathrm{C}$ correspondientes a la segunda. Las constantes de velocidad de enfriamiento fueron calculadas con la ecuación 12 (Resultados y Discusión sección 1). Se hallaron valores del orden de $10^{-1} \operatorname{seg}^{-1}$ para las fracciones enfriadas rápidamente y del orden de $10^{-3} \operatorname{seg}^{-1}$ para las enfriadas lentamente. Los resultados abtenidos se resumen en las Tablas 13, 14 y 15. La Tabla 13 corresponde a la muestra enfriada en forma lineal. La fracción cristalizada a $37^{\circ} \mathrm{C}$ presentó cinco soluciones sólidas diferentes en ambos casos (enfriamiento lento y rápido). La muestra enfriada rápidamente presentó un incremento del tercer pico respecto de la enfriada lentamente y una disminución del priner pico y del segundo. Las entalpias totales de estas muestras resultaron de 60,5 Joule/g y 60,7 Joule/g para las muestras enfriadas a $0,5^{\circ} \mathrm{C} / \mathrm{min}$ y $20^{\circ} \mathrm{C} / \mathrm{min}$ respectivamente, es decir se encontraron valores del mismo orden. Estos valores resultan ser inferiores a los informados en la sección 2.2 .3 para las fracciones sólidas superiores. Esto se debe a que en estas experiencias las corridas calorimétricos se comenzaron a $0^{\circ} \mathrm{C}$ mientras que cuando se anali- 
zaron las fracciones se comenzo a $-40^{\circ} \mathrm{C}$.

\section{Tabla 13}

Fracción $37^{\circ} \mathrm{C}$ enfriada con velocidad lineal

\begin{tabular}{|cccccr|}
\hline Temperatura $\left({ }^{\circ} \mathrm{C}\right)$ & 6,9 & 14,4 & 20,3 & 27,1 & 36,1 \\
Area de pico $(\%)$ & 11,9 & 23,7 & 9,3 & 33,5 & 21,7 \\
$\Delta \mathrm{H}$ (Joule/g) & 7,2 & 14,4 & 5,4 & 20,4 & 13,1
\end{tabular}

(2)

$\begin{array}{llcccc}\text { Temperatura }\left({ }^{\circ} \mathrm{C}\right) & 5,8 & 12,2 & 20,4 & 28,3 & 35,7 \\ \text { Area de pico }(\%) & 5,5 & 17,3 & 18,2 & 35,5 & 23,6 \\ \Delta H \text { (Joule/g) } & 3,3 & 10,5 & 11,1 & 21,5 & 14,3\end{array}$

(1) Eundida a $80^{\circ} \mathrm{C}$, enfriada a $0,5^{\circ} \mathrm{C} / \mathrm{min}$ y luego calentada de $0^{\circ} \mathrm{C}$ a $80^{\circ} \mathrm{C}$ a $10^{\circ} \mathrm{C} / \mathrm{min}$

(2) Fundida a $80^{\circ} \mathrm{C}$, enfriada a $20^{\circ} \mathrm{C} / \mathrm{min}$ y luego calentada de $0^{\circ} \mathrm{C}$ a $80^{\circ} \mathrm{C}$ \& $10^{\circ} \mathrm{C} / \mathrm{min}$ 
Las Tablas 14 y 15 corresponden a las muestras enfriadas en forma exponencial (primera y segunda experiencia respectivamente). Los valores de la Tabla 14 presentan también otros cambios. Además de lo ya explicado hay una disminución en el área del cuarto pico en las muestras enfriadas rápidamente respecto de las lentas y un corrimiento de los puntos de fusión hacia temperaturas menores. Esto nos dice que al aumentar la velocidad de criatalización el tiempo para reacomodarse en la forma más estable fue insuficiente. Los estudios realizados por difractometria de rayos $X$ no permitieron ver diferencias entre las fracciones rápidas y las lentas. Ambas presentaron diferentes tipos de cristales $\beta^{\prime}$. Cuando se calcularon las entalpias totales de estas muestras se encontraron valores de 29,$4 ; 38,7 ; 20,0$ y $16,3 \mathrm{~J}$ oule/g para las muestras enfriadas lentamente y 71,$6 ; 45,5 ; 40,3$ y 32,4 para las enfriadas rápidamente. 
Fracciones $41^{\circ} \mathrm{C}, \quad 35^{\circ} \mathrm{C}, 31^{\circ} \mathrm{C}$ y $21^{\circ} \mathrm{C}$ enfriadas con velocidad exponencial

Temp.

\begin{tabular}{|c|c|c|c|c|c|c|}
\hline \multirow[t]{3}{*}{41} & $\mathrm{~T}$ & 10,0 & 17,8 & 25,6 & 31,0 & 36,5 \\
\hline & $A$ & 4,7 & 24,2 & 33,0 & 33,1 & 5,1 \\
\hline & $\Delta \mathrm{H}$ & 1,4 & 7,1 & 9,7 & 9,7 & 1,5 \\
\hline \multirow[t]{3}{*}{35} & $\mathrm{~T}$ & 7,8 & 16,5 & 22,9 & 31,8 & \\
\hline & A & 26,8 & 18,8 & 24,0 & 30,4 & \\
\hline & $\Delta \mathrm{H}$ & 12,5 & 0,9 & 11,2 & 14,1 & \\
\hline \multirow[t]{3}{*}{31} & $\mathrm{~T}$ & 6,0 & 13,3 & 22,2 & 28,1 & \\
\hline & $A$ & 10,8 & 27,3 & 24,2 & 30,9 & \\
\hline & $\Delta \mathrm{H}$ & 4,7 & 0,7 & 6,4 & 8,2 & \\
\hline \multirow[t]{3}{*}{21} & 'T & 6,5 & 13,6 & 22,0 & 27,7 & \\
\hline & A & 17,5 & 20,9 & 26,7 & 34,9 & \\
\hline & $\Delta H$ & 2,8 & 3,4 & 4,4 & 5,7 & \\
\hline
\end{tabular}

T Temperatura de cristalización $\left({ }^{\circ} \mathrm{C}\right)$

A Erea de pico (\%)

$\Delta H$ entalpias de pico (Joule/g)

(a) Lentamente. Velocidad de enfriamiento $10^{-3} \mathrm{seg}^{-1}$ Temperatura final $0^{\circ} \mathrm{C}$ 
Temp.

\begin{tabular}{|c|c|c|c|c|c|c|}
\hline \multirow[t]{3}{*}{41} & $\mathrm{~T}$ & 9,8 & 17,0 & 28,5 & 31,6 & 35,5 \\
\hline & $A$ & 7,7 & 17,3 & 46,0 & 25,7 & 3,3 \\
\hline & $\Delta H$ & 5,5 & 12,3 & 33,0 & 18,4 & 2,4 \\
\hline \multirow[t]{3}{*}{35} & $\mathrm{~T}$ & 6,4 & 17,5 & 24,2 & 31,7 & \\
\hline & $A$ & 13,5 & 17,5 & 38,6 & 30,5 & \\
\hline & $\Delta H$ & 6,1 & 7,9 & 17,6 & 13,8 & \\
\hline \multirow[t]{3}{*}{31} & $\mathrm{~T}$ & 5,4 & 13,3 & 19,2 & 25,0 & \\
\hline & $A$ & 9,5 & 24,4 & 42,1 & 23,9 & \\
\hline & $\Delta H$ & 3,8 & 10,0 & 18,9 & 9,6 & \\
\hline \multirow[t]{3}{*}{21} & $\mathrm{~T}$ & 4,6 & 10,4 & 14,7 & 21,0 & \\
\hline & $A$ & 6,4 & 15,2 & 41,3 & 37,1 & \\
\hline & $\Delta H$ & 2,1 & 4,9 & 13,4 & 12,0 & \\
\hline
\end{tabular}

T' Temperatura $\left({ }^{\circ} \mathrm{C}\right)$

A Area de pico (\%)

$\Delta H$ entalpias de pico (Joule/g)

(b) Rápidamente. Velocidad de enfriamiento $10^{-1}$ seg-1 $^{-1}$

Temperatura final $0^{\circ} \mathrm{C}$

Las muestras pertenecientes a la segunda experiencia enfriadas rápidamente presentaron todas cinco soluciones sólidas (Tabla 15) al igual que las enfriadas lentamente con excepción de la 
muestra colectada a $36^{\circ} \mathrm{C}$ que presentó seis. Las muestras enfriadas rápidamente presentaron picos con temperaturas de fusión menores a las enfriadas lentamente. Las muestras lentas presentaron picos de temperatura de fusión cercana a la de cristalización en porcentajes mayores mientras que en las rápidas el último pico representó siempre el porcentaje mayor. Las muestras obtenidas en el equipo fraccionador resultaron poseor perfiles más semejantes a las fracciones cristalizadas lentamente lo cual era esperable. Cuando so calcularon las entalpias totales de las muestras se encontraron valores de 49,$2 ; 48,6$ y 41,3 Joule/g para las muestras enfriadas rápidamente y 53,$3 ; 55,9$ y 41,4 Joule/g para las muestras enfriadas lentamente. Tanto aqui como en el caso de enfriamiento con velocidad lineal los valores encontrados para ambas velocidades resultaron del orden. 
Tab Ia 15

Eracciones $36^{\circ} \mathrm{C}, 26^{\circ} \mathrm{C}$ y $16^{\circ} \mathrm{C}$ enfriadas con velocidad exponencial

Temp.

\begin{tabular}{|c|c|c|c|c|c|c|}
\hline \multirow[t]{3}{*}{36} & $\mathrm{~T}$ & 5,1 & 10,3 & 18,8 & 23,5 & 29,7 \\
\hline & $A$ & 10,3 & 15,2 & 13,5 & 27,0 & 34,0 \\
\hline & $\Delta \mathrm{H}$ & 5,1 & 7,5 & 6,6 & 13,3 & 16,7 \\
\hline \multirow[t]{3}{*}{26} & $\mathrm{~T}$ & 5,0 & 10,1 & 18,8 & 25,0 & 31,1 \\
\hline & A & 9,2 & 12,4 & 18,5 & 29,2 & 30,7 \\
\hline & $\Delta H$ & 4,5 & 6,0 & 8,0 & 14,2 & 14,9 \\
\hline \multirow[t]{3}{*}{16} & $\mathrm{~T}$ & 4,7 & 10,0 & 18,2 & 24,2 & 30,2 \\
\hline & $A$ & 10,3 & 14,1 & 19,1 & 29,6 & 26,9 \\
\hline & $\Delta H$ & 4,3 & 5,8 & 7,9 & 12,2 & 11,1 \\
\hline
\end{tabular}

(a) Rápidamente. Iguales condiciones que las empleadas para enfriar las muestras que figuran en la Tabla 14. $T$, A y $\Delta H$ poseen el mismo significado. 
Temp.

\begin{tabular}{|c|c|c|c|c|c|c|c|}
\hline \multirow[t]{3}{*}{36} & $\mathrm{~T}$ & 7,5 & 13,2 & 23,1 & 29,8 & 35,1 & 40,4 \\
\hline & A & 16,1 & 12,3 & 12,7 & 27,4 & 18,7 & 12,7 \\
\hline & $\Delta H$ & 8,6 & 6,6 & 6,8 & 14,6 & 10,0 & 6.7 \\
\hline \multirow[t]{3}{*}{26} & $\mathrm{~T}$ & 6,0 & 10,5 & 18,8 & 26,7 & 33,2 & \\
\hline & $A$ & 15,6 & 15,4 & 17,4 & 39,7 & 11,8 & \\
\hline & $\Delta H$ & 5,8 & 5,7 & 11,2 & 25,5 & 7,6 & \\
\hline \multirow[t]{3}{*}{16} & $\mathrm{~T}$ & 7,1 & 11,8 & 19,6 & 25,8 & 31,6 & \\
\hline & $A$ & 21,5 & 11,4 & 18,9 & 31,5 & 16,7 & \\
\hline & $\Delta H$ & 8,9 & 4,7 & 7,8 & 13,1 & 6,9 & \\
\hline
\end{tabular}

(b) Lentamente. Iguales condiciones que las empleadas para enfriar las muestras que figuran en la tabla 14

Si bien los constituyentes de las distintas muestras fueron ordenados en columnas, los diferentes picos posiblemente no posean idéntica composición quimica en todas las fracciones ya que cuando se analizaron las uismas asi como se extrajeron del tanque estas presentaron triglicéridos en porcentajes diferentes. En las tablas presentadas puede verse que los picos de menor temperatura presentan entalpias menores que los de mayor temperatura. Por esta razón podriamos esperar que los triglicéridos insaturados de menor calor de fusión fueran los responsables de los primeros picos y los trigliceridos saturados de los otros.

Schlichter y colaboradores (1988) han señalado que para una sustancia dada existe usualmente una relación lineal entre las 
temperaturas de fusión $y$ sus correspondientes entalpias. Sin embargo a ciertas velocidades de enfriamiento las entalpias observadas son menores que las esperadas. A velocidades de cristalización lentas los valores de las entalpias no se incrementan como una función de los puntos de fusión. Esto puede deberse a que no se obtenga una forma polimórfica definida o a que durante la cristalización ocurra segregación. En nuestro sistema nosotros no encontramos linealidad entre puntos de fusión y entalpias. La Tabla 16 resume las temperaturas, áreas por ciento y entalpias halladas cuando se fundib a diferentes velocidades la fracción de $26^{\circ} \mathrm{C}$ (segunda experiencia). La Figura 29 compara las interpretaeiones hechas en la computadora de los termogramas empleados para construir la Tabla 16. Se observa como habiamos mencionado que los valores de las entalpias no se incrementan como una función de la temperatura. Por otra parte los puntos de fusión de los tres primeros constituyentes son mayores en las fracciones fundidas y enfriadas con velocidades de $2^{\circ} \mathrm{C}, \quad 5^{\circ} \mathrm{C}$ y $20^{\circ} \mathrm{C}$ que en la muestra extraida del tanque mientras que los dos picos de temperaturas superiores tienen puntos de fusión inferiores. Las entalpias de cada constituyente en las distintas muestras son diferentes. Dado que la composición quimica de todas las muestras es la misma, cada solución sólida estaria formada por diferentes triglicéridos en distintas proporciones según la posibilidad de difundir de las distintos triglicéridos. 
Tabla 16

Calorinetria diferencial de barrido do la fracción $26^{\circ} \mathrm{C}$ asi cono fue obtenida y fundida y enfriada a distintas velocidades

Temp.

\begin{tabular}{|c|c|c|c|c|c|c|}
\hline 26 & $T$ & 3,8 & 10,4 & 16,2 & 27,5 & 33,7 \\
\hline & A & 7,2 & 25,7 & 21,1 & 24,2 & 19,8 \\
\hline & $\Delta \mathrm{H}$ & 3,3 & 11,8 & 9,7 & 11,1 & 9,2 \\
\hline 26 & $\mathrm{~T}$ & 5,9 & 12,3 & 18,6 & 24,6 & 32,4 \\
\hline Enf.a & A & 11,3 & 25,3 & 10,7 & 26,2 & 26,5 \\
\hline $2^{\circ} \mathrm{C} / \mathrm{min}$ & $\Delta \mathrm{H}$ & 5,8 & 13,0 & 5,5 & 13,4 & 13,6 \\
\hline 26 & $\mathrm{~T}$ & 6,8 & 12,4 & 19,4 & 25,5 & 32,1 \\
\hline Enf $a$ & A & 16,3 & 14,8 & 18,4 & 21,5 & 29,0 \\
\hline $5^{\circ} \mathrm{C} / \mathrm{min}$ & $\Delta \mathrm{H}$ & 7,6 & 6,9 & 8,6 & 10,0 & 13,5 \\
\hline 26 & $T$ & 6,2 & 12,2 & 18,0 & 25,8 & 32,4 \\
\hline Enf.a & A & 4,8 & 17,0 & 23,7 & 29,8 & 24,7 \\
\hline $20^{\circ} \mathrm{C} / \mathrm{min}$ & $\Delta H$ & 2,4 & 8,6 & 12,0 & 15,1 & 12,6 \\
\hline
\end{tabular}

T Temperatura $\left({ }^{\circ} \mathrm{C}\right)$

A Area de pico (\%)

$\Delta H$ entalplas de pico (Joule/g) 


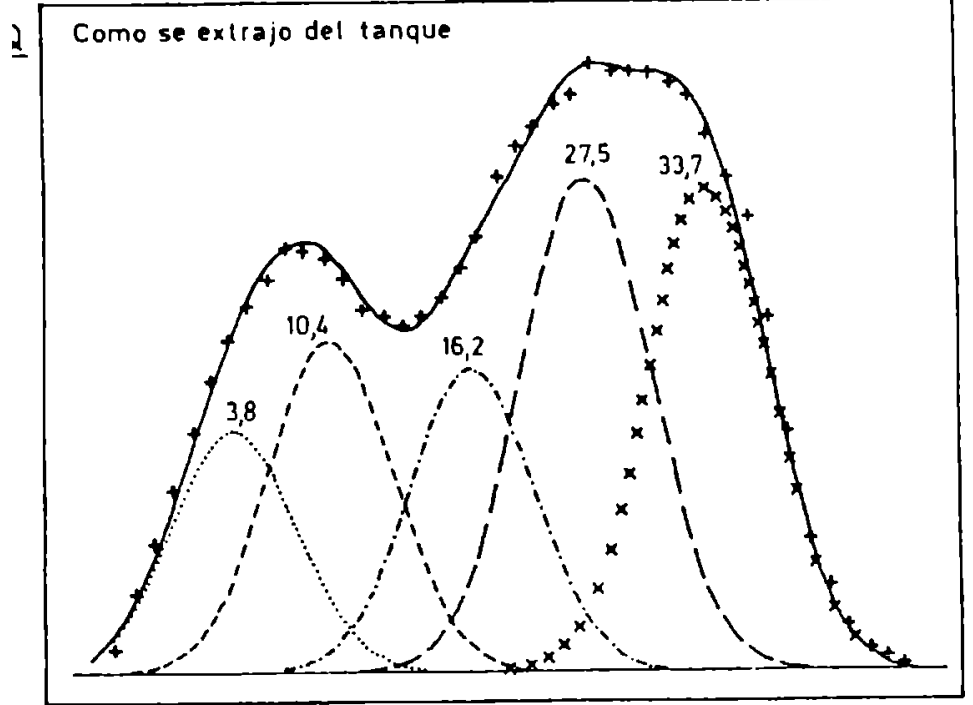

tiempo (min)

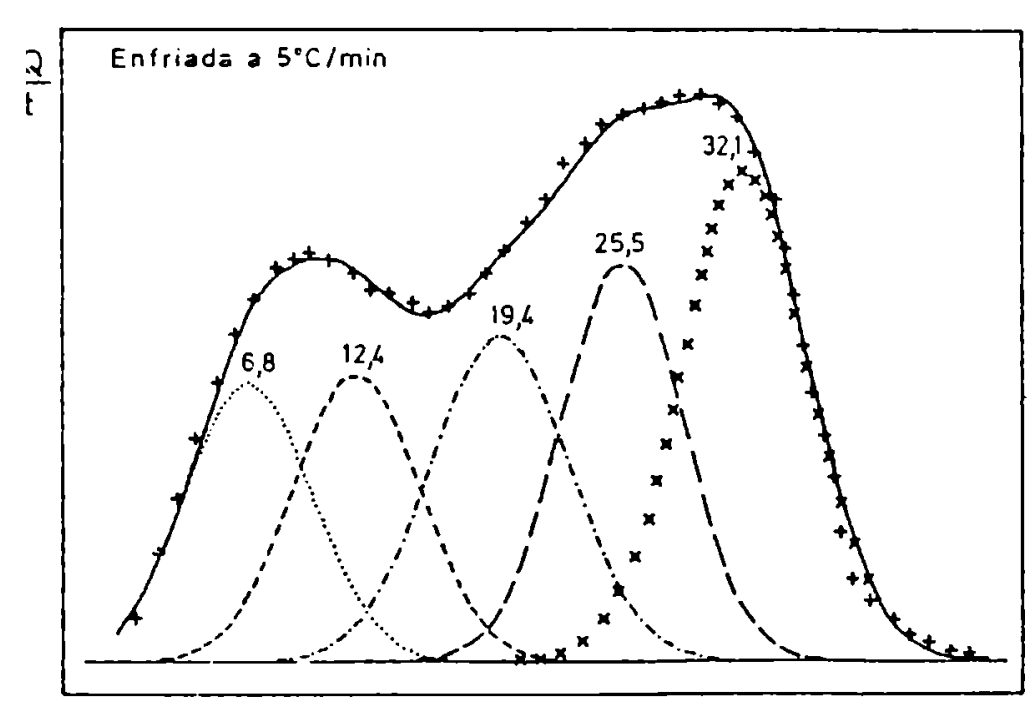

tiempo $(\mathrm{min})$
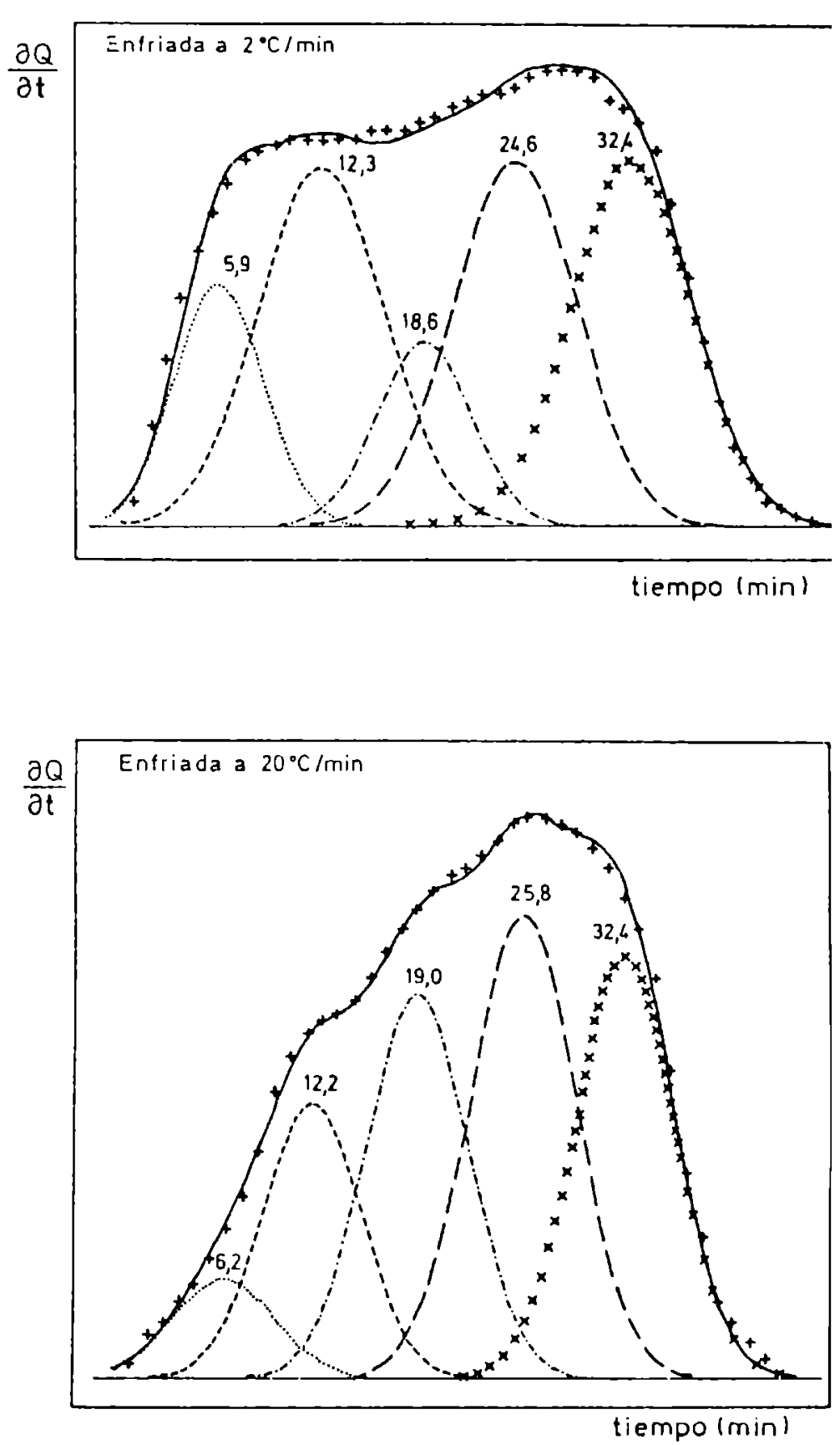

Figura 29. Interpretación por conputadora de los termogranas de la muestra $26^{\circ} \mathrm{C}$ cono se extrajo del tanque y fundida y enfriada a distintas velocidades 
Conclusiones 
Los resultados obtenidos en este trabajo muestran gue: - Ia cristalización fraccionada permite la separación de mezclas de triglicéridos complejas tales como los aceites hidrogenados.

- los cortes sólidos obteridos a temperaturas superiores a $31^{\circ} \mathrm{C}$ están constituidos principalmente por triglicéridos de número equivalente de carbonos alto (SSO, SOO, POO). Alrededor del $40 \%$ de estos triglicéridos son isómeros trans. Cuando se estudiaron estas muestras por calorimetria diferencial de barrido los isómeros cis y trans de estos triglicéridos presentaron puntos de fusión muy diferentes apareciendo como dos constituyentes en el termograma mientras que en el cromatograma eluyen en un único pico. Los diagramas calorimétricos de estas muestras resultaron más anchos que los correspondientes a las fracciones sólidas obtenidas a quenores temperaturas observándose una contribución mayor a la endoterma total de los constituyentes de mayor punto de fusión.

- los cortes sólidos obtenidos entre $31^{\circ} \mathrm{C}$ y $23^{\circ} \mathrm{C}$ presentaron triglicéridos con tiempos de retención intermedios especialmente se enriguecen en el triglicérido 000. Si bien el porcentaje de isómeros trans es del mismo orden que el del grupo anterior los contenidos de ácido oleico fueron menores; por consiguiente la masa de isómeros trans en este grupo resulto inferior al anterior. Los diagramas calorimétricos presentaron porcentajes menores de componentes de alto punto de fusión.

- las muestras obtenidas por debajo de $23^{\circ} \mathrm{C}$ presentaron contenidos altos de triglicéridos de número equivalente de carbonos menor, en 
este grupo se registró un incremento importante de ácido linoleico. Si bien el contenido de isómeros trans resultó importante los diagramas calorinétricos fueron más angostos y las entalpias totales menores que las de las muestras incluidas en los otros grupos.

- las muestras liquidas presentaron mayores contenidos en sus triglicéridos de ácidos grasos insaturados que los detectados en las fracciones sólidas cristalizadas a la misma tenperatura. Partiendo de la composición en triglicéridos de la muestra original se registraron cambios pequeños y graduales. Los porcentajes de isóneros trans resultaron mucho menores que en los sólidos. En concordancia con esto los diagramas calorimétricos obtenidos fueron más angostos y las entalpias totales menores que en los sólidos correspondientes.

Los equilibrios planteados entre sólidos y liquidos nos permiten calcular con una aproximación razonable la composición aproximada en triglicéridos a partir de un contenido de sólidos dado. De 10 expuesto surge que en el fraccionamiento de aceite de girasol hidrogenado los sólidos retienen una porción de liquido debido a :

-que dada la naturaleza electroquimica y tamaño similar de los triglicéridos, triglicéridos insaturados o isóneros cis ocupen sitios en la red correspondientes a triglicéridos saturados o isómeros trans.

- los cristales de grasa pueden retener liquido en su estructura. 
Bibliografia 
Abu-Hadeed A.M. and A.R. Kotb, A Method for the Quantitative Determination of Individual Oils in a Blend, J. Am. Oil Chem. Soc. $65: 1922(1988)$

Amer M.A., D.B. Kupranycz and B.E. Baker, Physical and Chemical Characteristics of Butterfat Fractions Obtained by Crystallization from Motten Fat, J. Am. Oil Chem. Soc. 62:1551 (1985).

Apraiz Barreiro J., Tratamientos tórmicos de los aceros, Editorial Dossat, Madrid (1964).

Arul J., A. Boudreau, J. Makhlouf, R. Tardif and T. Bellavia, Fractionation of Anhydrous Milk Eat by Short-Path Distillation, $J$. Am. Oil Chem. Soc. 65:1642 (1988).

Azoury R., J.S. Aronhime, S. Sarig, S. Abrashkin, I. Mayer and N. Garti, NMR Relaxation Studies to Explore the Role of Emulsifier in Tristearin Polymorphic Transformation, J. Am. Oil Chem. Soc. $65: 964(1988)$.

Bailey A.E., Melting and Solidification of Fats, Intersec. Publ. Pag 4, New York (1850).

Bociek S.M., S. Ablett and I.T. Norton, A 13C-NMR Study of the Crystal Polymorphism and Internal Mobilities of the Triglycerides, Tripaluitin and Triestearin, J. Am. Oil Chem. Soc. 62:1261 (1985). 
Chapman D., Infrared Spectra and the Polymorphism of Glycerides. Part III. Palmitodistearins and Dipalmitostearins, J. Chem. Soc. $2715(1957)$

Chapman D., The $720 \mathrm{~cm}-1$ Band in the Infrared Spectra of Crystalline Long-Chain Compounds, J. Chem. Soc. 4489 (1957).

Chapman D., Infrared Spectroscopic Characterization of Glycerides, J. Am. oil Chem. Soc. $37: 73$ (1960).

Charbonret G.H. and W.S. Singleton, Thermal Properties of Fats and Oils. VI Heat caprcity, Heats of Fusion and Transition, and Entropy of Trilaurin, Trimyristin and Tristearin, J. Am. Oil Chem Soc. $24: 140(1947)$.

Chikamy B., Los procesos de cristalización de las grasas y su papel en la producción de margarinas I, Olaj, Szappan, Rozmetika $1: 102(1965)$.

Chikamy B., Los procesos de cristalización de las grasas y su papel en la producción de margarinas II, 0laj, Szappan, Kozmetika $2: 9(1966)$.

Chikary B., Los procesos de cristalización de las grasas y su papel en la producción de margarinas III, 0laj, Szappan, Kozmetika $3: 52(1967)$. 
Uafler J.R., Polymorphism Behavior in Fully Hydrogenated Mono Acid Triglycerides, J. Am. Oil Chem. Soc. 54:249 (1977).

Deffense E., Fractionation of Palm Oil, J. Am. Oil Chem. Soc. $63: 376(1985)$.

Deroanne C., Contribution à l'étude de la cristallisation des glycérides. Application au fractionnement de l'huile de palme, Thèse de Doctorat, Facultés des Sciences Agronomiques de l'Etat, Gembloux, Belgique (1974).

Deroanne C., Structure Studies on the Triglycerides of Palm Oil Eractions. I- Development of a Method of Analysis for Triglycerides of the Solid and Liquid Palm Oil Eractions, Revue Francaise des Corps Gras 22:599 (1975).

Deroanne C., Structure Studies on the Triglycerides of Palm Oil Eractions. II- Development of a Method of Analysis for Triglycerides of the Solid and Liquid Palm Oil Fractions, Revue Francaise des Corps Gras 22:11 (1975).

Deroanne C., Triglyceride Structure of Fractionated Palm Oil. IThe Course of Triglyceride Crystallization during Cooling of Palm Oil for the Tirtiaux Fractionation Process, Revue Francaise des Corps: Gras 23:1 (1976). 
Deroanne C., Triglyceride Structure of Fractionated Palm Oil. IIThe Course of Trigliceride Crystallization during Cooling of Palm Oil for the Tirtiaux Fractionation Process, Revue Francaise des Corps Gras $23: 27$ (1976).

Deroanne C., La filtration dars le raffinage et le fractionement des Corps Gras, Symposium International, C.E.R.I.A. Bruxelles, 2829, avri1, 1976 .

Deroanne C., Le Polymorphisme et le Fractionnement Sans Solvant de 1'Huile de Palme son Influence sur la Filtration, 13ème Congrès Mondial de I'I.S.F. Marseille, Septembre 1976.

Deroanne C., Characterization of the Intersolubility of the Glycerides of Palm Oil, Lebensm. Wiss. U. Technol. 9:304 (1976).

Deroanne C., Differential Scanning Calorimetry, its Practical Interest for the Fractionation of Palm Oil and the Determination of the Solid Fat Index, Lebensm. Wiss. U. Technol. 10:251 (1977).

Deroanne $C$. La double cristallisation de l'huile de palme mise en evidence par difraction des rayons $X$ et analyse calorimetrique differentielle, Journal of Thermal Analysis 11:109 (1977).

Diccionario Enciclopédico Monitor, Editorial Salvat, Londres, 1960 
Dimick P.S. and D.M. Manning, Thermal and Compositional Properties of Cocoa Butter During Static Crystallization, J. Am. Oil Chem. Soc. $64: 1663(1987)$.

Done J.N. J.H. Knox and J. Loheac, Applications of High-Speed Liquid Chromatography, John Wiley and sons Ltd, England, 1874.

Dong and Di Cesare, Improved Separation of Natural Oil Triglicerides by Liquid Chromatography Using Columns Packed with 3 Micrometres Particles, J. Am. Oil Chem. Soc. 60:788 (1983).

Donovan J.W. and K.D. Ross, Iron Binding to Conalbumin, Journal of Biological Chemistry 250:6026 (1975).

Drachmann A.G., Ancient Oil Mills and Presses, Kgl Danske Videnskabernes Selskab, Copenhagen, 1932.

Eckey E.W., Vegetable Eats and Oils, Reinhold Pub. Corp., New York, 1954 .

Frede E. and H. Thiele, Analysis of Milkfat by HPLC, J. Am. Oil Chem. Soc. $64: 521$ (1987).

Garti N. and K. Sato, Effects of Surfactants on Transition Kinetics of Stearic Acid Polymorphs, J. Am. Oil Chem. Soc. 63:236 (1986). 
Garti N.. J. Schlichter and S. Sarig, Effect of Food Emulsifiers on Polymorphic Transitions of Cocoa Butter, J. Am. Oil Chem. Soc. $63: 230(1986)$.

Graciani Constante E., Caracterización del aceite de oliva virgen español. I-Estudio de las variables que intervienen en la separación de sus triacilgliceroles por cromatografia liquida de alta eficacia, Grasas y aceites $38: 286$ (1987).

Gibon V., F. Durant and C. Deroanne, Polymorphisu and Intersolubility of Some Palmitic, Stearic and Oleic Triglycerides PPP, PSP and POP, J. Am. Oil Chem. Soc. 63:1047 (1886).

Glastone S., Tratado de quimica fisica, Aguilar, New York, 1949.

Haighton A.J., Blending, Chilling and Tempering of Margarine and Shortenings, J. Am. Oil Chem. Soc. 53:397 (1976).

Hamilton R.J. and Bhati A., Fats and Oils: Chemistry and Technology, Applied Science Publishers, London, 1980.

Hampsion J.W. and H.L. Rothbart, Triglyceride Specific Heat Determined by Differential Scanning Calorimetry, J. Am. Oil Chen. Soc. $60: 1102(1983)$.

Hinnekens H., Fractionation of Lipids without the Use of a Solvent. "In Eiltration in the Refining and Fractionation of Oils and Fats" 145-170 (1976). 
Jacobsberg B., Studies in Palm Oil Crystallization, J. Am. Oil Chem. Soc., 53:609 (1976).

Jacobsberg B., Aims and Methods of Fractionation of Fats in Filtration in the Refining and Fractionation of $O i l s$ and Fats Pag. $119-143(1976)$.

Jacqmain D., Limitations on Storage Time of Frozen Foods, Revue des Fermentations et des Industries Alimentaires $32(2) \quad 40-45$ $(1977)$.

Jamieson G.S., Vegetable Fats and Oils, Reinhold Pub. Corp., New York, 1942 .

Jaroslov N., Industrial Crystallization from Solutions, Edition Butterworths, 1971 .

Johnson E.L. and R. Stevenson, Basic Liquid Chromatography, Varian, E.E.U.U., 1978.

Kimmey R.L. and E.G. Perkings, Confectionery Fat Analysis with High Perfomance Liquid Chromatography, J. Am. Oil Chem. Soc. $61: 1209$ ( 1984$)$.

Knoester M., P. De Bruyne and M. Van Den Tempel, Crystallization of Triglycerides at Low Supercooling, Journal of crystal Growth, $3,4,776-780(1968)$. 
Kroester M., P. De Bruyne and M. Van Den Tempel, The Solid-Liquid Equilibrium of Binary Mixtures of Triglycerides with Palmitic and Stearic chains, Chem. Phys. Lipids, 9:303 (1972).

Kochhar S.P., and J.B. Rosse11, Determination of 0 il in Sunflowerseeds, J. Am. 0il Chem. Soc., 64:865 (1987).

Kreulen H.P., Fractionation and Winterization of Edible Fats and Oils, J. Am. Oil Chem. Soc. 53:393 (1976).

Larsson K., Solid States Behaviour of Glycerides, Arkiv. Kemi., $23: 35(1964)$.

Lutton E.S., Phase Behavior of Triglyceride Mixtures Involving Primarily Tristearin, 2-Oleyldistearin and Triolein, J. Am. Oil Chem. Soc., $32: 49$ (1955).

Lutton E.S., Binary Systems from Palmitic-Stearic Triglycerides, J. Am. Oil Chem. Soc., 44:303 (1967).

Lutton E.S., Lipid Structures (A lecture), J. Am. Oil Chem. Soc., $49: 1(1972)$.

Madsen J., Changes in Margarine and Bakery Compound Caused by Physical Transformations in the Fat Phase during Storage, Zeszyty Problemowe Postepow Nauk Rolniczych, 136:147 (1973). 
Malkin T.. Progress in the Chemistry of Fats and other Lipids, Pergamon Press LTD, Pag 1-50, London, 1954.

Merker D.R., L.C. Brown and L.H. Wiedermann, The Relationship of Polimorfism to the Texture of Margarine Containing Soybean and Cottonseed Oils, J. Am. Oil Chem. Soc., 35:130 (1958).

Mozior C., J.M. de Man and L. de Man, Effect of Tempering on the Physical Properties of Shortening, Journal of Canadian Institute of Food Science and Technology 22:238 (1989).

Naguib-Mostafa A. and J.M. de Man, Polymorphism of Hydrogenated Canola Oil, J. Am. Oil Chem.Soc.62:756 (1985).

Naguit-Mostafa A., A.K. Smith and J.M. de Man, Crystal Structure of Hydrogenated Canola Oil, J. Am. Oil Chem. Soc. 62:760 (1985).

Nawar W.W., Food Chemistry, Edited by O.R. Fennema pag. 148 (1985).

Norton I.T., C.D. Lee-Tuffnell, S. Ablett and S.M. Bociek, A Calorimetric, NMR and X-Ray Diffraction Study of the Melting Behavior of Tripalmitin and Tristearin and their Mixing Behavior with Triolein, J. Am. Oil Chem. Soc. 62:1237 (1985).

Ong A.S.H., P.L. Boey and C.M. Ong, Fractionation Studies of Palm 0il by Density Gradient, J. Am. 0il Chem. Soc. 61:1755 (1983). 
Kaulicka F.R., Phase Behaviour of Cocoa Butter Extenders, Chem. and Ind. 1/9/73, Pag. 835 .

Perron R.R., Essai de Prévision du Comportement Thermique d une Graisse en Fonction de sa Composition Glycéridique I- Principe et Elaboration de la methode, Revue Erancaise des Corps Gras 33:195 (1986).

Perron R.R., Comentaires sur le Comportement Thermique des Triglycerides. Róle de I'Insaturation, Revue Francaise des Corps Gras $31: 171(1984)$.

Perron R.R. et M. Broncy, Comportement Thermique des Matières Grasses I- Huile de Palme et Produits Dérivés, Revue Francaise des Corps Gras 25:165 (1978).

Perron R.R. et M. Broncy, Comportement Thermique des Matières Grasses II- Huile de Colza et Produits Dérivés, Revue Erancaise des Corps Gras 25:525 (1978).

Persmark U., R.A. Melin, P.O. Stahl, Analysis of Rapeseed Oil, ISF Congress, Milano pag. 174 (1974).

Riiner U., The Effect of Hydrolysis on the Solidification of Fats, Lebensawiss. U. Technol., 4:76 (1971).

Rossell J.B., Interactions of Triglycerides and of Fats Containing them, Chem. and Ind. 1/9/73 Pag. 832 . 
Rossell J.B., Fractionation of Lauric Oils, J. Am. Oil Chem. Soc. $63: 385$ (1985).

Sato K., and M. Suzuki, Solvent Crystallization of $\alpha, \beta$ and Polimorphs of Oleic Acid, J. Am. Oil Chem. Soc. 63:1356 (1986).

Schlichter J., N. Garti and S. Sarig, Heat Capacity of Tristearin in the Presence of Food Emulsifiers, J. Am. Oil Chem. Soc. 63:788 (1986).

Schlichter J., J.S. Aronhime, S. Sarig and N. Garti, Mechanistic Considerations of Polymorphic Transformations of Tristearin in the Presence of Emulsifiers, J. Am. 0il Chem. Soc. 64:529 (1987).

Schlichter J., J.S. Aronhime, S. Sarig and N. Garti, Reconsideration of Polymorphic Transformations in Cocoa Butter Using the DSC, J. Am. 0il Chem. Soc. 65:1140 (1988).

Schwitzer M.K., Margarine and other food fats, Leonard Hill Limited, London, 1951.

Sebedio J.L., C.H. Septier and A. Grandgirard, Fractionation of Commercial Frying Oil Samples using Sep-Pak Cartridges, J. Am. Oil Chem. Soc. 63:1541(1986)

Segura J.A., M.L. Herrera and M.C. Añón, Storage of Commercial Margarine, J. Am. Oil Chem. Soc. (En prensa). 
Simpson T.D. and J.W. Hagemann, Crystallographic Study of Tritetracosanoin, J. Am. 0 il Chem. Soc., $52: 303$ (1975).

Simpson T.D., Solid Phases of Trimargarin: A Comparison to Tristearin, J. Am. 0il Chem. Soc. 60:95 (1983).

Simpson T.D., D.P. Hockett and L. Harris, Specific Heats of the Solid-State Phases of Trimargarin and Tristearin, J. Am. Oil Chem. Soc. $61: 883(1984)$.

Singleton J.A. and Pattee H.W. Optimization of Parameters for the Analysis of Triglycerides by Reverse Phase HPLC Using a JV Detector at $210 \mathrm{~nm}, J$. Am. 0il Chem. Soc. 81:761 (1984).

Suzuki M., P. Ogaki and K. Sato, Crystallization and Transformation Mechanisms of $\alpha, \beta$ and Polymorphs of Ultra-Pure Oleic Acid, J. Am. Oil Chem. Soc. 62:1600 (1985).

Takahashi K., T. Hirano and K. Zama, A New Concept for Determining Triglyceride Composition of Fats and Oils by Liquid Chromatography, J. Am. Oil Chem. Soc. 61:1226 (1984).

Takahashi K., T. Hirano, M. Egi and K. Zama, A Mathematical Model for the Prediction of Triglycerides Molecular Species by High Perfomance Liquid Chromatography, J. Am. 0il Chem. Soc. 62:1489 (1985). 
Takahashi K., T. Hirano, M. Egi M. Hatano and K. Zama, Supplementary Consideration of the Triglyceride Matrix Model on Reverse Phase High Perfomance Liquid Chromatography, J. Am. Oil Cheat. Soc. 63:1543 (1986).

Takano S. and $Y$. Kondoh, A Method for the Quantitative Determination of Individual Oils in a Blend, J.Am. Oil Chem. Soc. $65: 1922(1988)$

Traitter H., H.J. Wille and A. Studer Fractionation of Blackcurrant Seed Oil, J. Am. Oil Chem. Soc. 65:755 (1988).

Van Den Tempel M., Effect of Emulsifiers on the Crystallization of Triglycerides, S.C.I. Monograph 32, 22-33 (1968).

Vsn der Hoek W., Application d'une Méthode Nouvelle à l'étude de 1a. Stabilité Structurale de la Margarina, Revue Francaise des Corps Gras 12:761 (1969).

Van Futte K.P.A.M. and B.H. Bakker, Crystallization Kinetics of Palm 0il, J. Am. Oil Chem. Soc. 64:1138 (1987).

Von Speisefetten D.U., Gemeinschaftsarbeit Der Deutschen Gesellschaft Fur Fettwissenschaft e.v. (1973) Fette, Seifen, Anstrichm., 75, 467, 587 and 663 .

Walker W., Aging of Tristearin: Comparison of DSC and Positron Lifetime Results, J. Am. Oil Chem. Soc. 64:754 (1987). 
Wille R.L. and E.S. Lutton, Polymorphism of Cocoa Butter, J. Am. Oil Chem. Soc. $43: 491$ (1968).

Wood R. and Lee T., High-Eerfomance Liquid Chromatography of Fatty Acids: Quantitative Analysis of Saturated, Monoenoic, Polyenoic and Geometrical Isomers, J. Chromatog. $254: 237$ (1983).

Zajis J., L. Ferman, J. Korma, Anderung Verschiedener Eigenschaften Von'Fetten Wahrend der Desodorisierung, Fette, Seifen und Anstrichmittel, 70:860 (1968).

Zschau M.R., Hydrogenation Catalysts, Preparation, Physical Eroperties, Practical Applications, C.E.R.I.A., Bruxelles 28-29 Avril (1976)

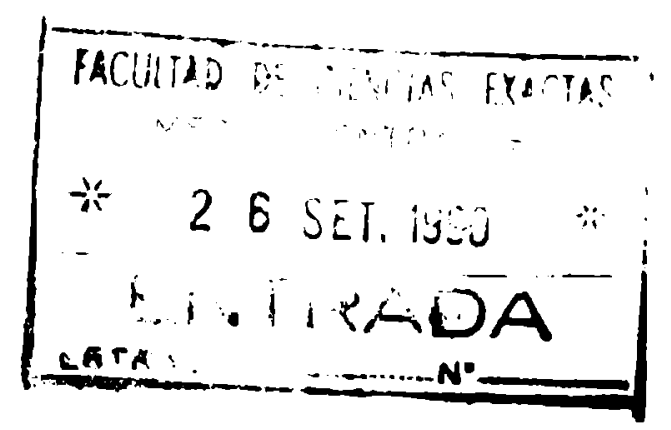


- Los espaciamientos cortos y largos en los diagramas de rayos $X$ se expresaron en $A$, debe decir $\dot{A}$.

- La acidez fue expresada en porcentaje de ácidos grasos libres. La norma indica que debe expresarse en mg de $\mathrm{KoH} / \mathrm{g}$.

- Dado que nuestros métodos analiticos (excepto la espectroscopia de infrarrojol no son capaces de distinguir entre los distintos isómeros geométricos y de posición de los ácidos grasos y triglicéridos si no se especifica lo contrario cuando dice ácido oleico se refiere a todos los isómeros C18:1 y cuando dice ácido linoleico se refiere a todos los isómeros c18:2.

- Pag. 14 Dice: encontraron que las fases $\alpha$ y $\beta$ de trimargarina... Debe decir: fases $\alpha$ y de triheptadecanoína...

- Pag. 31 Neutralización: posibilita la eliminación de ácidos grasos, pigmentos... Debe decir: ácidos grasos libres, pigmentos... 